\title{
The Beurling-Lax-Halmos Theorem for Infinite Multiplicity
}

October 14, 2019

\author{
Raúl E. Curto \\ In Sung Hwang \\ Woo Young Lee
}





\section{Contents}

Abstract vii

Chapter 1. Introduction 1

Chapter 2. Preliminaries 9

Chapter 3. Strong $L^{2}$-functions 13

Chapter 4. The Beurling-Lax-Halmos Theorem 27

Chapter 5. A canonical decomposition of strong $L^{2}$-functions 45

Chapter 6. The Beurling degree 53

Chapter 7. The spectral multiplicity of model operators 59

Chapter 8. Miscellanea 73

Chapter 9. Some unsolved problems 83

Bibliography 89 



\begin{abstract}
In this paper, we consider several questions emerging from the Beurling-LaxHalmos Theorem, which characterizes the shift-invariant subspaces of vector-valued Hardy spaces. The Beurling-Lax-Halmos Theorem states that a backward shiftinvariant subspace is a model space $\mathcal{H}(\Delta) \equiv H_{E}^{2} \ominus \Delta H_{E}^{2}$, for some inner function $\Delta$. Our first question calls for a description of the set $F$ in $H_{E}^{2}$ such that $\mathcal{H}(\Delta)=E_{F}^{*}$, where $E_{F}^{*}$ denotes the smallest backward shift-invariant subspace containing the set $F$.

In our pursuit of a general solution to this question, we are naturally led to take into account a canonical decomposition of operator-valued strong $L^{2}$-functions. This decomposition reduces to the Douglas-Shapiro-Shields factorization if the flip of the strong $L^{2}$-function is of bounded type. (Given a strong $L^{2}$-function $\Phi$, we define its flip by $\breve{\Phi}(z):=\Phi(\bar{z})$.)

Next, we ask: Is every shift-invariant subspace the kernel of a (possibly unbounded) Hankel operator? As we know, the kernel of a Hankel operator is shiftinvariant, so the above question is equivalent to seeking a solution to the equation ker $H_{\Phi}^{*}=\Delta H_{E^{\prime}}^{2}$, where $\Delta$ is an inner function satisfying $\Delta^{*} \Delta=I_{E^{\prime}}$ almost everywhere on the unit circle $\mathbb{T}$ and $H_{\Phi}$ denotes the Hankel operator with symbol $\Phi$.

Consideration of the above question on the structure of shift-invariant subspaces leads us to study and coin a new notion of "Beurling degree" for an inner function. We then establish a deep connection between the spectral multiplicity of the model operator, i.e., the truncated backward shift on the corresponding model space, and the Beurling degree of the corresponding characteristic function.

At the same time, we consider the notion of meromorphic pseudo-continuations of bounded type for operator-valued functions, and then use this notion to study the spectral multiplicity of model operators (truncated backward shifts) between separable complex Hilbert spaces. In particular, we consider the case of multiplicityfree: more precisely, for which characteristic function $\Delta$ of the model operator $T$ does it follow that $T$ is multiplicity-free, i.e., $T$ has multiplicity 1 ? We show that if $\Delta$ has a meromorphic pseudo-continuation of bounded type in the complement of the closed unit disk and the adjoint of the flip of $\Delta$ is an outer function then $T$ is multiplicity-free.

In the case when the characteristic function $\Delta$ of the model operator $T$ has a finite-dimensional domain (in particular, when $\Delta$ is an inner matrix function) admitting a meromorphic pseudo-continuation of bounded type in the complement of the closed unit disk, we prove that the spectral multiplicity of $T$ can be computed from that of the induced $C_{0}$-contraction, and as a result the characteristic function is two-sided inner. Finally, by using the preceding results we analyze left and right
\end{abstract}


coprimeness, the model operator, and an interpolation problem for operator-valued functions.

2010 Mathematics Subject Classification. Primary 46E40, 47B35, 30H10, 30J05; Secondary 43A15, 47A15

Key words. The Beurling-Lax-Halmos Theorem, strong $L^{2}$-functions, a canonical decomposition, the Douglas-Shapiro-Shields factorization, a complementary factor of an inner function, the degree of non-cyclicity, functions of bounded type, the Beurling degree, the spectral multiplicity, the model operator, characteristic inner functions, meromorphic pseudo-continuation of bounded type, multiplicity-free.

The work of the second named author was supported by NRF(Korea) grant No. 2019R1A2C1005182. The work of the third named author was supported by NRF(Korea) grant No. 2018R1A2B6004116.

Affiliations:

Raúl E. Curto: Department of Mathematics, University of Iowa, Iowa City, IA 52242, U.S.A. email:raul-curto@uiowa.edu

In Sung Hwang: Department of Mathematics, Sungkyunkwan University, Suwon 440-746, Korea, email: ihwang@skku.edu

Woo Young Lee: Department of Mathematics, Seoul National University, Seoul 151-742, Korea, email: wylee@snu.ac.kr 


\section{CHAPTER 1}

\section{Introduction}

The celebrated Beurling Theorem $\mathbf{B e u}$ characterizes the shift-invariant subspaces of the Hardy space. P.D. Lax $[\mathbf{L a x}]$ extended the Beurling Theorem to the case of finite multiplicity, and proved the so-called Beurling-Lax Theorem. Subsequently, P.R. Halmos Ha1 gave a beautiful proof for the case of infinite multiplicity, and thus established the so-called Beurling-Lax-Halmos Theorem. Since then, the Beurling-Lax-Halmos Theorem has been extended to various settings and extensively applied in connection with model theory, system theory and the interpolation problem by many authors (cf. [ADR, AS, BH1, [BH2, [BH3, $\mathbf{B a}$, dR, [Hed], [Po, [Ri], [SFBK]).

In this paper, we will focus on a detailed analysis of the Beurling-Lax-Halmos Theorem for infinite multiplicity. We obtain answers to several questions emerging from the classical Beurling-Lax-Halmos Theorem and establish some new and exciting results, including: (i) a canonical decomposition for operator-valued $L^{2}$ functions (in fact, for a much bigger class of functions), (ii) the introduction of the Beurling degree of an inner function, and (iii) the study of the spectral multiplicity of a model operator.

Let $\mathbb{T}$ be the unit circle in the complex plane $\mathbb{C}$. Throughout this paper, whenever we deal with operator-valued functions $\Phi$ on $\mathbb{T}$, we assume that $\Phi(z)$ is a bounded linear operator between separable complex Hilbert spaces for almost all $z \in \mathbb{T}$. For a separable complex Hilbert space $E$, let $S_{E}$ be the shift operator on the $E$-valued Hardy space $H_{E}^{2}$, i.e.,

$$
\left(S_{E} f\right)(z):=z f(z) \text { for each } f \in H_{E}^{2} .
$$

The Beurling-Lax-Halmos Theorem states that every subspace $M$ invariant under $S_{E}$ (i.e., a closed subspace of $H_{E}^{2}$ such that $S_{E} f \in M$ for all $f \in M$ ) is of the form $\Delta H_{E^{\prime}}^{2}$, where $E^{\prime}$ is a closed subspace of $E$ and $\Delta$ is an inner function. As usual, $\Delta$ is an inner function if $\Delta(z)$ is an isometric operator from $E^{\prime}$ into $E$ for almost all $z \in \mathbb{T}$, i.e., $\Delta^{*} \Delta=I_{E^{\prime}}$ a.e. on $\mathbb{T}$. If, in addition, $\Delta \Delta^{*}=I_{E}$ a.e. on $\mathbb{T}$, then $\Delta$ is called a two-sided inner function.

There exists an equivalent description of a closed subspace $M$ of $H_{E}^{2}$ which is invariant under the backward shift operator $S_{E}^{*}$; that is, $M=\mathcal{H}(\Delta):=H_{E}^{2} \ominus \Delta H_{E^{\prime}}^{2}$ for some inner function $\Delta$. The space $\mathcal{H}(\Delta)$ is often called a model space or a de Branges-Rovnyak space $\mathbf{d R},\left[\mathbf{S a},\left[\mathbf{S F B K}\right.\right.$. Thus, for a subset $F$ of $H_{E}^{2}$, if $E_{F}^{*}$ denotes the smallest $S_{E}^{*}$-invariant subspace containing $F$, i.e.,

$$
E_{F}^{*}:=\bigvee\left\{S_{E}^{* n} F: n \geq 0\right\},
$$

(where $\bigvee$ denotes the closed linear span), then $E_{F}^{*}=\mathcal{H}(\Delta)$ for some inner function $\Delta$.

Now, given a backward shift-invariant subspace $\mathcal{H}(\Delta)$, we may ask: 
QUESTION 1.1. (i) What is the smallest number of vectors in $F$ satisfying $\mathcal{H}(\Delta)=E_{F}^{*}$ ?

(ii) More generally, we are interested in the problem of describing the set $F$ in $H_{E}^{2}$ such that $\mathcal{H}(\Delta)=E_{F}^{*}$.

To examine Question 1.1 we need to consider (possibly unbounded) linear operators (defined on the unit circle) constructed by arranging the vectors in $F$ as column vectors. In other words, in what follows we will encounter bounded linear operators whose "column" vectors are $L^{2}$-functions. (Since bounded linear operators between separable Hilbert spaces can be represented as infinite matrices, considering the columns of such a matrix as column vectors of the operator seems well justified). This approach naturally leads to the notion of (operator-valued) strong $L^{2}$-function. This notion seems to have been introduced by V. Peller $\mathbf{P e}$, Appendix 2.3] for the purpose of defining general symbols of vectorial Hankel operators. However, Pellers book gives only the definition of a strong $L^{2}$-function, and does not describe the properties of such functions. Besides Pellers book, we have not found any other references in the literature to strong $L^{2}$-functions. In Chapter 3 we study strong $L^{2}$-functions (including operator-valued $L^{2}$ - and $L^{\infty}$-functions) and then derive some basic properties.

Let $\mathcal{B}(D, E)$ denote the set of all bounded linear operators between separable complex Hilbert spaces $D$ and $E$. A strong $L^{2}$-function $\Phi$ is a $\mathcal{B}(D, E)$-valued function defined almost everywhere on the unit circle $\mathbb{T}$ such that $\Phi(\cdot) x \in L_{E}^{2}$ for each $x \in D$. We can easily see that every operator-valued $L^{p}$-function $(p \geq 2)$ is a strong $L^{2}$-function (cf. p 13). Following V. Peller $\mathbf{P e}$, we write $L_{s}^{2}(\mathcal{B}(D, E)$ ) for the set of strong $L^{2}$-functions with values in $\mathcal{B}(D, E)$.

The set $L_{s}^{2}(\mathcal{B}(D, E))$ constitutes a nice collection of general symbols of vectorial Hankel operators (see $[\mathbf{P e})$. Similarly, we write $H_{s}^{2}(\mathcal{B}(D, E)$ ) for the set of strong $L^{2}$-functions with values in $\mathcal{B}(D, E)$ such that $\Phi(\cdot) x \in H_{E}^{2}$ for each $x \in D$. Of course, $H_{s}^{2}(\mathcal{B}(D, E))$ contains all $\mathcal{B}(D, E)$-valued $H^{2}$-functions. In Chapter 3, we study operator-valued Hardy classes as well as strong $L^{2}$-functions as a groundwork of this paper.

Question 1.1 is closely related to a canonical decomposition of strong $L^{2}$ functions. We first observe that if $\Phi$ is an operator-valued $L^{\infty}$-function, then the kernel of the Hankel operator $H_{\Phi^{*}}$ is shift-invariant. Thus by the BeurlingLax-Halmos Theorem, the kernel of the Hankel operator $H_{\Phi^{*}}$ is of the form $\Delta H_{E^{\prime}}^{2}$ for some inner function $\Delta$. If the kernel of the Hankel operator $H_{\Phi^{*}}$ is trivial, take $E^{\prime}=\{0\}$. Of course, $\Delta$ need not be a two-sided inner function. In fact, we can show that if $\Phi$ is an operator-valued $L^{\infty}$-function and $\Delta$ is a two-sided inner function, then the kernel of the Hankel operator $H_{\Phi^{*}}$ is $\Delta H_{E^{\prime}}^{2}$ if and only if $\Phi$ is expressed in the form

$$
\Phi=\Delta A^{*},
$$

where $A$ is an operator-valued $H^{\infty}$-function such that $\Delta$ and $A$ are right coprime (see Lemma 4.2). The expression (1.1) is called the (canonical) Douglas-ShapiroShields factorization of an operator-valued $L^{\infty}$-function $\Phi$ (see [DSS], [FB], Fu2]; in particular, Fu2 contains many important applications of the Douglas-ShapiroShields factorization to linear system theory).

Let $\mathbb{D}$ be the open unit disk in the complex plane $\mathbb{C}$. We recall that a meromorphic function $\varphi: \mathbb{D} \rightarrow \mathbb{C}$ is said to be of bounded type (or in the Nevanlinna class) if 
it is a quotient of two bounded analytic functions. A matrix function of bounded type is defined by a matrix-valued function whose entries are all of bounded type. Very recently, a systematic study on matrix-valued functions of bounded type was undertaken in the research monograph CHL3. It is also known that every matrixvalued $L^{\infty}$-function whose adjoint is of bounded type satisfies (1.1) (cf. GHR). In fact, if we extend the notion of "bounded type" for operator-valued $L^{\infty}$-functions (as we will do in Definition 4.18 for a bigger class), then we may say that the expression (1.1) characterizes the class of $L^{\infty}$-functions whose flips are of bounded type, where the flip $\breve{\Phi}$ of $\Phi$ is defined by $\breve{\Phi}(z):=\Phi(\bar{z})$. From this viewpoint, we may ask whether there exists an appropriate decomposition corresponding to general $L^{\infty}$-functions, more generally, to strong $L^{2}$-functions. The following problem is the first objective of this paper,

Problem 1.2. Find a canonical decomposition of strong $L^{2}$-functions.

To establish a canonical decomposition of strong $L^{2}$-functions, we need to introduce new notions; this will be done in Chapter 4. First of all, we coin the notion of "complementary factor", denoted by $\Delta_{c}$, of an inner function $\Delta$ with values in $\mathcal{B}(D, E)$. This notion is defined by using the kernel of $\Delta^{*}$, denoted by ker $\Delta^{*}$, which is defined by the set of vectors $f$ in $H_{E}^{2}$ such that $\Delta^{*} f=0$ a.e. on $\mathbb{T}$. Moreover, the kernel of $H_{\Delta^{*}}$ can be represented by orthogonally adding the complementary factor $\Delta_{c}$ to $\Delta$ (see Lemma 4.5) . We also employ a notion of "degree of non-cyclicity" on the set of all subsets (or vectors) of $H_{E}^{2}$, which is a complementary notion of "degree of cyclicity" due to V.I. Vasyunin and N.K. Nikolskii [VN]. The degree of non-cyclicity, denoted by $\mathrm{nc}(F)$, of subsets $F \subseteq H_{E}^{2}$, is defined by the number

$$
\operatorname{nc}(F):=\sup _{\zeta \in \mathbb{D}} \operatorname{dim}\left\{g(\zeta): g \in H_{E}^{2} \ominus E_{F}^{*}\right\} .
$$

Thus, in comparison with the degree of cyclicity, the degree of non-cyclicity admits $\infty$, which is often beneficial when trying to understand the Beurling-Lax-Halmos Theorem. Now, for a canonical decomposition of strong $L^{2}$-functions $\Phi$, we are tempted to guess that $\Phi$ can be factored as $\Delta A^{*}$ (where $\Delta$ is a possibly onesided inner function) as in the Douglas-Shapiro-Shields factorization, in which $\Delta$ is two-sided inner. But this is not the case. In fact, we can see that a canonical decomposition is actually affected by the kernel of $\Delta^{*}$ through some examples (see p. 45). Upon reflection, we recognize that this is not an accident. This is accomplished in Chapter 5.

Theorem 5.1 realizes the idea inside those examples: if $\Phi$ is a strong $L^{2}$-function with values in $\mathcal{B}(D, E)$, then $\Phi$ can be expressed in the form

$$
\Phi=\Delta A^{*}+B,
$$

where $\Delta$ is an inner function with values in $\mathcal{B}\left(E^{\prime}, E\right), \Delta$ and $A$ are right coprime, $\Delta^{*} B=0$, and $\operatorname{nc}\left\{\Phi_{+}\right\} \leq \operatorname{dim} E^{\prime} . \quad\left(\left\{\Phi_{+}\right\}\right.$denotes the set of all "column" vectors of the analytic part of $\Phi$ ). In particular, if $\operatorname{dim} E^{\prime}<\infty$ (for instance, if $\operatorname{dim} E<\infty$ ), then the expression (1.3) is unique (up to a unitary constant right factor) (see Theorem [5.1, p. 46). The expression (1.3) will be called a canonical decomposition of a strong $L^{2}$-function $\Phi$. The proof of Theorem 5.1 shows that the inner function $\Delta$ in the canonical decomposition (1.3) of a strong $L^{2}$-function $\Phi$ can be obtained from the equation

$$
\operatorname{ker} H_{\breve{\Phi}}^{*}=\Delta H_{E^{\prime}}^{2}
$$


which is guaranteed by the Beurling-Lax-Halmos Theorem (see Corollary 4.4). In this case, the expression (1.3) will be called the BLH-canonical decomposition of $\Phi$, recalling that $\Delta$ comes from the Beurling-Lax-Halmos Theorem. However, if $\operatorname{dim} E^{\prime}=\infty$ (even in the case when $\operatorname{dim} D<\infty$ ), then it is possible to get another inner function $\Theta$ of a canonical decomposition (1.3) for the same function: in this case, $\operatorname{ker} H_{\overparen{\Phi}}^{*} \neq \Theta H_{E^{\prime \prime}}^{2}$. Therefore the canonical decomposition of a strong $L^{2}$ function is not unique in general (see Remark 5.2). But the second assertion of Theorem 5.1 says that if the codomain of $\Phi(z)$ is finite-dimensional (in particular, if $\Phi$ is a matrix-valued $L^{2}$-function), then the canonical decomposition (1.3) of $\Phi$ is unique; in other words, the inner function $\Delta$ in (1.3) should be obtained from the equation ker $H_{\overparen{\Phi}}^{*}=\Delta H_{E^{\prime}}^{2}$. Thus the unique canonical decomposition (1.3) of matrix-valued $L^{2}$-functions is precisely the BLH-canonical decomposition.

Further, if the flip $\breve{\Phi}$ of $\Phi$ is of bounded type then $B$ turns to be a zero function, so that the decomposition (1.3) reduces to the Douglas-Shapiro-Shields factorization. In fact, the Douglas-Shapiro-Shields factorization was given for $L^{\infty}$-functions, but the case $B=0$ in (1.3) is available for strong $L^{2}$-functions. Moreover, the notion of "bounded type" for matrix-valued functions is not appropriate for operatorvalued functions, i.e., the statement "each entry of the matrix is of bounded type" does not produce a natural extension to operator-valued functions even though it has a meaning for infinite matrices (remember that we deal with operators between separable Hilbert spaces).

Thus we need to introduce an appropriate notion of "bounded type" for operatorvalued functions. We will do this in Section 4.4. Moreover, to guarantee the statement "each entry is of bounded type," we adopt the notion of "meromorphic pseudo-continuation of bounded type" in $\mathbb{D}^{e}:=\{z: 1<|z| \leq \infty\}$, which coincides with the notion of "bounded type" for matrix-valued functions (cf. Fu1): This will be done in Section 4.5.

On the other hand, we recall that the spectral multiplicity for a bounded linear operator $T$ acting on a separable complex Hilbert space $E$ is defined by the number $\mu_{T}$ :

$$
\mu_{T}:=\inf \operatorname{dim} F,
$$

where $F \subseteq E$, the infimum being taken over all generating subspaces $F$, i.e., subspaces such that $M_{F} \equiv \bigvee\left\{T^{n} F: n \geq 0\right\}=E$. In the definition of the spectral multiplicity, $F$ may be taken as a subset rather than a subspace. In this case, we may regard $\mu_{T}$ as the quantity $\inf \operatorname{dim} \bigvee\{f: f \in F\}$ such that $M_{F}=E$. Unless this leads to ambiguity, we will deal with $M_{F}$ for subsets $F \subseteq E$. If $S_{E}$ is the shift operator on $H_{E}^{2}$, then it is known that $\mu_{S_{E}}=\operatorname{dim} E$. By contrast, if $S_{E}^{*}$ is the backward shift operator on $H_{E}^{2}$, then $S_{E}^{*}$ has a cyclic vector, i.e., $\mu_{S_{E}^{*}}=1$. Moreover, the cyclic vectors of $S_{E}^{*}$ form a dense subset of $H_{E}^{2}$ (see [Ha4, [Ni1, Wo). We here observe that Question 1.1(i) is identical to the problem of finding the spectral multiplicity of the truncated backward shift operator $\left.S_{E}^{*}\right|_{\mathcal{H}(\Delta)}$, i.e., the restriction of $S_{E}^{*}$ to its invariant subspace $\mathcal{H}(\Delta)$. The second objective of this paper is to show that this problem has a deep connection with a canonical decomposition of strong $L^{2}$-functions involved with the inner function $\Delta$.

To understand the smallest $S_{E}^{*}$-invariant subspace containing a subset $F \subseteq H_{E}^{2}$, we need to consider the kernels of the adjoints of unbounded Hankel operators with strong $L^{2}$-symbols involved with $F$. Thus we will deal with unbounded Hankel operators $H_{\Phi}$ with strong $L^{2}$-symbols $\Phi$. However, the adjoint of the unbounded 
Hankel operator need not be a Hankel operator. But if $\Phi$ is an $L^{\infty}$-function then $H_{\Phi^{*}}=H_{\breve{\Phi}}^{*}$, where $\breve{\Phi}$ is the flip of $\Phi$. Thus for a bounded symbol $\Phi$, we may use the notations $H_{\Phi^{*}}$ and $H_{\widetilde{\Phi}}^{*}$ interchangeably. By contrast, for a strong $L^{2}$-function $\Phi, H_{\Phi^{*}}$ may not be equal to $H_{\overparen{\Phi}}^{*}$ even though $\Phi^{*}$ is a strong $L^{2}$-function. In particular, the kernel of an unbounded Hankel operator $H_{\Phi^{*}}$ is likely to be trivial because it is defined on the dense subset of polynomials. From this viewpoint, to avoid potential technical issues in our arguments, we will deal with the operator $H_{\Phi}^{*}$ in place of $H_{\Phi^{*}}$. In spite of this, and since the kernel of the adjoint of an unbounded operator is always closed, we can show that via the Beurling-Lax-Halmos Theorem, the kernel of $H_{\overparen{\Phi}}^{*}$ with strong $L^{2}$-symbol $\Phi$ is still of the form $\Delta H_{E^{\prime}}^{2}$ (see Corollary 4.4).

We now consider several questions, which are of independent interest. This will be done in Chapter 4. The next question arises naturally from the BeurlingLax-Halmos Theorem.

QUESTION 1.3. Since the kernel of the Hankel operator $H_{\overleftrightarrow{\Phi}}^{*}$ is of the form $\Theta H_{E^{\prime}}^{2}$, which property of $\Phi$ determines the dimension of the space $E^{\prime}$ ? In particular, if $\Phi$ is an $n \times m$ matrix-valued $L^{2}$-function and $\operatorname{dim} E^{\prime}=r$, which property of $\Phi$ determines the number $r$ ?

To answer Question 1.3, we employ the notion of degree of non-cyclicity (1.2). Indeed, we can show that if the kernel of the adjoint of the Hankel operator $H_{\breve{\Phi}}$ is $\Theta H_{E^{\prime}}^{2}$ for some inner function $\Theta$, then the dimension of $E^{\prime}$ can be computed by the degree of non-cyclicity of $\left\{\Phi_{+}\right\}$(see Theorem 4.10). Here we note that the definition of $\left\{\Phi_{+}\right\}$depends on the orthonormal bases of the domain $D$ of $\Phi(\cdot)$. However, the degree of non-cyclicity of $\left\{\Phi_{+}\right\}$is independent of the particular choice of orthonormal basis of $D$ (see Theorem 4.10).

When $\Delta$ is an inner function, we may ask when it is possible to complement $\Delta$ to a two-sided inner function by aid of an inner function $\Omega$; in other words, when is $[\Delta, \Omega]$ a two-sided inner function, where $[\Delta(\cdot), \Omega(\cdot)]$ is understood as an $1 \times 2$ operator matrix defined on the unit circle $\mathbb{T}$ ? (It turns out that this question can be answered by using the Complementing Lemma; see [VN or Ni1). The following question refers to more general cases.

QUESTION 1.4. If $\Delta$ is an $n \times r$ inner matrix function, which condition on $\Delta$ allows us to complement $\Delta$ to an $n \times(r+q)$ inner matrix function using an $n \times q$ inner matrix function?

An answer to Question 1.4 is also subject to the degree of non-cyclicity of $\{\Delta\}$ (see Corollary 4.16).

By the Beurling-Lax-Halmos Theorem, we saw that the kernel of the adjoint of a Hankel operator with a strong $L^{2}$-symbol is of the form $\Delta H_{E^{\prime}}^{2}$ for some inner function $\Delta$. In view of its converse, we may ask:

QUESTION 1.5. Is every shift-invariant subspace $\Delta H_{E^{\prime}}^{2}$ represented by the kernel of $H_{\overparen{\Phi}}^{*}$ with some strong $L^{2}$-symbol $\Phi$ with values in $\mathcal{B}(D, E)$ ?

Question 1.5 asks whether a strong $L^{2}$-solution $\Phi$ always exists for the equation ker $H_{\overparen{\Phi}}^{*}=\Delta H_{E^{\prime}}^{2}$ for a given inner function $\Delta$. In Theorem 6.1 we give an affirmative answer to Question 1.5. The matrix-valued version of this result is as follows (see Corollary 6.2): for a given $n \times r$ inner matrix function $\Delta$, there always exists a 
solution $\Phi \in L_{M_{n \times m}}^{\infty}$ of the equation $\operatorname{ker} H_{\overparen{\Phi}}^{*}=\Delta H_{\mathbb{C}^{r}}^{2}$, for some $m \leq r+1$. In view of this, it is reasonable to ask whether such a solution $\Phi \in L_{M_{n \times m}}^{2}$ exists for each $m=1,2, \cdots$. But the answer to this question is negative (see Remark 6.4).

It is then natural to ask how to determine a possible dimension of $D$ for which there exists a strong $L^{2}$-solution $\Phi$ (with values in $\mathcal{B}(D, E)$ ) of the equation ker $H_{\overparen{\Phi}}^{*}=\Delta H_{E^{\prime}}^{2}$. In fact, we would like to ask what is the infimum of $\operatorname{dim} D$ that guarantees the existence of a strong $L^{2}$-solution $\Phi$. To find a way to determine such an infimum, we introduce the notion of "Beurling degree" for an inner function. We do this by employing the canonical decomposition of a strong $L^{2}$ function induced by the given inner function: if $\Delta$ is an inner function with values in $\mathcal{B}\left(E^{\prime}, E\right)$, then the Beurling degree, denoted by $\operatorname{deg}_{B}(\Delta)$, of $\Delta$ is defined by the infimum of the dimension of the nonzero space $D$ for which there exists a pair $(A, B)$ such that $\Phi \equiv \Delta A^{*}+B$ is a canonical decomposition of a strong $L^{2}$-function $\Phi$ with values in $\mathcal{B}(D, E)$ (Definition $[6.5)$.

We now recall that the Model Theorem ([Ni1, SFBK] ) states that if a bounded operator $T$ acting on a Hilbert space $\mathcal{H}$ (in symbols, $T \in \mathcal{B}(\mathcal{H})$ ) is a contraction (i.e., $\|T\| \leq 1$ ) satisfying

$$
\lim _{n \rightarrow \infty} T^{n} x=0 \quad \text { for each } x \in \mathcal{H}
$$

then $T$ is unitarily equivalent to a truncated backward shift $\left.S_{E}^{*}\right|_{\mathcal{H}(\Delta)}$ for some inner function $\Delta$ with values in $\mathcal{B}\left(E^{\prime}, E\right)$, where $E=\operatorname{cl} \operatorname{ran}\left(I-T^{*} T\right)$. In this case, $\left.S_{E}^{*}\right|_{\mathcal{H}(\Delta)}$ is called the the model operator of $T$ and $\Delta$ is called the characteristic function of $T$. We often write $T \in C_{0}$. for a contraction operator $T \in \mathcal{B}(\mathcal{H})$ satisfying the condition (1.4).

We can now prove that if $\Delta$ is the characteristic function of the model operator $T$ with values in $\mathcal{B}\left(E^{\prime}, E\right)$, with $\operatorname{dim} E^{\prime}<\infty$ (in particular, when $\Delta$ is an inner matrix function), then the spectral multiplicity of the model operator is equal to the Beurling degree of $\Delta$. Equivalently, given an inner function $\Delta$ with values in $\mathcal{B}\left(E^{\prime}, E\right)$, with $\operatorname{dim} E^{\prime}<\infty$, let $T:=\left.S_{E}^{*}\right|_{\mathcal{H}(\Delta)}$. Then

$$
\mu_{T}=\operatorname{deg}_{B}(\Delta)
$$

(see Theorem 6.6). The equality (1.5) is the second objective of this paper. It is somewhat surprising that the spectral multiplicity of the model operator can be computed by a function-theoretic property of the corresponding characteristic function.

The third objective of this paper is to consider the case of $\mu_{T}=1$, i.e., when the operator $T$ has a cyclic vector. In general, if $T \in \mathcal{B}(\mathcal{H})$ is such that $\mu_{T}=1$, then $T$ is said to be multiplicity-free. To avoid confusion, we regard $T$ to be multiplicity-free if the operator $T$ acts on the zero space. Thus we are interested in the following question on the characteristic function $\Delta$ of $T$.

Question 1.6. Let $T:=\left.S_{E}^{*}\right|_{\mathcal{H}(\Delta)}$. For which inner function $\Delta$ does it follow that $T$ is multiplicity-free?

To get an answer to Question [1.6, we consider the notion of "characteristic scalar" inner function, which is a generalization of the case of two-sided inner matrix function (and we often call it square inner matrix function) (cf. [Hel, SFBK, CHL3). This will be done in Section 7.1. If $\Delta$ is an inner function and $\Delta_{c}$ is its complementary factor, we write $\Delta_{c c} \equiv\left(\Delta_{c}\right)_{c}, \Delta_{c c c} \equiv\left(\Delta_{c c}\right)_{c}, \cdots$, etc. for 
the successive iterated complementary factors of $\Delta$. The key idea for an answer to Question 1.6 is given in the following result. First, let $\widetilde{\Delta}(z):=\Delta(\bar{z})^{*}$.

If an inner function $\Delta$ has a meromorphic pseudo-continuation of bounded type in $\mathbb{D}^{e}$ and if $\widetilde{\Delta}$ is an outer function, then $\Delta_{c c}=\Delta$ (see Lemma 7.13).

We can then get an answer to Question [1.6] as follows:

If $T:=\left.S_{E}^{*}\right|_{\mathcal{H}(\Delta)}$, where $\Delta$ has a meromorphic pseudo-continuation of bounded type in $\mathbb{D}^{e}$ and $\widetilde{\Delta}$ is an outer function, then $T$ is multiplicity-free (see Theorem 7.14).

Recall that for an inner matrix function $\Delta$, the condition " $\Delta$ has a meromorphic pseudo-continuation of bounded type" in $\mathbb{D}^{e}$ is equivalent to the condition " $\breve{\Delta}$ is of bounded type" (see Corollary 4.27). As a consequence, the matrix-valued version of Theorem 7.14 can be rephrased as follows: If $\Delta$ is an inner matrix function whose flip $\breve{\Delta}$ is of bounded type and if $\Delta^{t}$, the transpose of $\Delta$, is an outer function, then $T:=\left.S_{E}^{*}\right|_{\mathcal{H}(\Delta)}$ is multiplicity-fee (see Corollary 7.15). We may ask whether the converse of the key idea (Lemma 7.13) for Theorem 7.14 is true; i.e., if $\Delta$ is an inner function having a meromorphic pseudo-continuation of bounded type in $\mathbb{D}^{e}$ and $\Delta_{c c}=\Delta$, does it follow that $\widetilde{\Delta}$ is an outer function? We can show that the answer to this question is affirmative when $\Delta$ is an inner matrix function: i.e., if $\Delta_{c c}=\Delta$, then $\widetilde{\Delta}$ is an outer function when $\Delta$ is an inner matrix function whose flip $\breve{\Delta}$ is of bounded type (see Corollary 7.16).

On the other hand, the theory of spectral multiplicity for $C_{0}$-operators has been well developed in terms of their characteristic functions (cf. [Ni1, Appendix 1]). However this theory is not applied directly to $C_{0}$-operators, in which cases their characteristic functions need not be two-sided inner. The fourth objective of this paper is to show that if the characteristic function of a $C_{0}$-operator $T$ has a finite-dimensional domain and a meromorphic pseudo-continuation of bounded type in $\mathbb{D}^{e}$, then its spectral multiplicity can be computed by that of the $C_{0}$-operator induced by $T$. This will be done in Section 7.3. The main theorem of that section is as follows: Given an inner function $\Delta$ with values in $\mathcal{B}\left(E^{\prime}, E\right)$, with $\operatorname{dim} E^{\prime}<\infty$, let $T:=\left.S_{E}^{*}\right|_{\mathcal{H}(\Delta)}$. If $\Delta$ has a meromorphic pseudo-continuation of bounded type in $\mathbb{D}^{e}$, then

$$
\mu_{T}=\mu_{T_{s}}
$$

where $T_{s}$ is a $C_{0}$-contraction of the form $T_{s}:=\left.S_{E^{\prime}}^{*}\right|_{\mathcal{H}\left(\Delta_{s}\right)}$ with $\Delta_{s}:=\widetilde{(\widetilde{\widetilde{\Delta}})^{i}}$. Hence in particular, $\mu_{T} \leq \operatorname{dim} E^{\prime}$. (Here $(\cdot)^{i}$ means the inner part of the inner-outer factorization of the given $H^{\infty}$-function.) (see Theorem 7.24).

In Theorem [7.24 we note that $\Delta_{s} \equiv \widetilde{(\widetilde{\Delta})^{i}}$ is a two-sided inner function (see Lemma 7.21) (and hence, $T_{s}$ belongs to the class $C_{0}$ ). Therefore (1.6) shows that the spectral multiplicity of a $C_{0}$-operator can be determined by the induced $C_{0^{-}}$ operator if its characteristic function has a meromorphic pseudo-continuation of bounded type in $\mathbb{D}^{e}$. On the other hand, it was known (cf. [Ni1, p. 41]) that if $T:=\left.S_{E}^{*}\right|_{\mathcal{H}(\Delta)}$ for an inner function $\Delta$ with values in $\mathcal{B}\left(E^{\prime}, E\right)$, with $\operatorname{dim} E^{\prime}<$ $\operatorname{dim} E$, then

$$
\mu_{T} \leq \operatorname{dim} E^{\prime}+1
$$


if further $\operatorname{dim} E^{\prime}=\operatorname{dim} E<\infty$, then

$$
\mu_{T} \leq \operatorname{dim} E^{\prime} .
$$

Thus, the equation (1.6) shows that (1.8) still holds without the assumption $\operatorname{dim} E^{\prime}=$ $\operatorname{dim} E$.

The organization of this paper is as follows. The main theorems of this paper are Theorem 5.1 (a canonical decomposition of strong $L^{2}$-functions), Theorem 6.6 (the Beurling degree and the spectral multiplicity), Theorem 7.14 (multiplicity-free model operators), and Theorem 7.24 (the spectral multiplicity of model operators). To prove those theorems, we need to consider several questions emerging from the Beurling-Lax-Halmos Theorem. We also consider several auxiliary lemmas, and new notions of complementary factors of inner functions, the degree of non-cyclicity, bounded type strong $L^{2}$-functions, and the Beurling degree of an inner function.

In Chapter 2 we give the notations and the basic definitions. In Chapter 3 we study operator-valued strong $L^{2}$-functions and then prove some properties which will be used in the sequel. In Section 4.1-4.3 we introduce notions of complementary factors of inner functions and the degree of non-cyclicity, and then give answers to Question 1.3 and Question 1.4. In Section 4.4 we introduce the notion of "bounded type" strong $L^{2}$-functions, which correspond to the functions whose entries are of bounded type in the matrix-valued case.

In Chapter 5 we establish a canonical decomposition of a strong $L^{2}$-functions $\Phi$, which reduces to the Douglas-Shapiro-Shields factorization of $\Phi$ if $\breve{\Phi}$ is of bounded type. In Chapter 6 we give an answer to Question 1.5 and then establish a connection between the spectral multiplicity of the model operator and the Beurling degree of the corresponding characteristic function.

In Chapter 7 we consider the spectral multiplicity of model operators by using the notion of meromorphic pseudo-continuation of bounded type in the complement of the closed unit disk and then give an answer to Question [1.6. In Chapter 8 by using the preceding results, we analyze the left and right coprimeness, the model operator and an interpolation problem for operator-valued functions. In Chapter 9 we address some unsolved problems. 


\section{CHAPTER 2}

\section{Preliminaries}

In this chapter we provide notations and definitions, which will be used in this paper.

We write $\mathbb{D}$ for the open unit disk in the complex plane $\mathbb{C}$ and $\mathbb{T}$ for the unit circle in $\mathbb{C}$. To avoid a confusion, we will write $z$ for points on $\mathbb{T}$ and $\zeta$ for points in $\mathbb{C} \backslash \mathbb{T}$. For $\phi \in L^{2}$, write

$$
\breve{\phi}(z):=\phi(\bar{z}) \quad \text { and } \quad \widetilde{\phi}(z):=\overline{\phi(\bar{z})} .
$$

For $\phi \in L^{2}$, write

$$
\phi_{+}:=P_{+} \phi \quad \text { and } \quad \breve{\phi}_{-}:=P_{-} \phi,
$$

where $P_{+}$and $P_{-}$are the orthogonal projections from $L^{2}$ onto $H^{2}$ and $L^{2} \ominus H^{2}$, respectively. Thus, we may write $\phi=\breve{\phi}_{-}+\phi_{+}$.

Throughout the paper, we assume that

\section{$X$ and $Y$ are complex Banach spaces;}

$D$ and $E$ are separable complex Hilbert spaces.

We write $\mathcal{B}(X, Y)$ for the set of all bounded linear operators from $X$ to $Y$ and abbreviate $\mathcal{B}(X, X)$ to $\mathcal{B}(X)$. For a complex Banach space $X$, we write $X^{*}$ for its dual. We write $M_{n \times m}$ for the set of $n \times m$ complex matrices, and abbreviate $M_{n \times n}$ to $M_{n}$. We also write g.c.d.(.) and l.c.m.(.) denote the greatest common inner divisor and the least common inner multiple, respectively, while left-g.c.d.(.) and left-l.c.m. $(\cdot)$ denote the greatest common left inner divisor and the least common left inner multiple, respectively.

If $A: D \rightarrow E$ is a linear operator whose domain is a subspace of $D$, then $A$ is also a linear operator from the closure of the domain of $A$ into $E$. So we will only consider those $A$ such that the domain of $A$ is dense in $D$. Such an operator $A$ is said to be densely defined. If $A: D \rightarrow E$ is densely defined, we write $\operatorname{dom} A$, $\operatorname{ker} A$, and $\operatorname{ran} A$ for the domain, the kernel, and the range of $A$, respectively. If $A: D \rightarrow E$ is densely defined, write

$$
\operatorname{dom} A^{*}=\{e \in E:\langle A d, e\rangle \text { is a bounded linear functional for all } d \in \operatorname{dom} A\} .
$$

Then there exists a unique $f \in E$ such that $\langle A d, e\rangle=\langle d, f\rangle$ for all $d \in \operatorname{dom} A$. Denote this unique vector $f$ by $f \equiv A^{*} e$. Thus $\langle A d, e\rangle=\left\langle d, A^{*} e\right\rangle$ for all $d \in$ $\operatorname{dom} A$ and $e \in \operatorname{dom} A^{*}$. We call $A^{*}$ the adjoint of $A$. It is well known from unbounded operator theory (cf. Go, $\mathbf{C o n}$ ) that if $A$ is densely defined, then $\operatorname{ker} A^{*}=(\operatorname{ran} A)^{\perp}$, so that $\operatorname{ker} A^{*}$ is closed even though ker $A$ may not be closed.

We recall ( $\underline{\mathbf{A b}}, \mathbf{C o 2}, \mathbf{G H R}, \mathbf{N i 1})$ that a meromorphic function $\phi: \mathbb{D} \rightarrow \mathbb{C}$ is said to be of bounded type (or in the Nevanlinna class $\mathcal{N}$ ) if there are functions 
$\psi_{1}, \psi_{2} \in H^{\infty}$ such that

$$
\phi(z)=\frac{\psi_{1}(z)}{\psi_{2}(z)} \quad \text { for almost all } z \in \mathbb{T} .
$$

It is well known that $\phi$ is of bounded type if and only if $\phi=\frac{\psi_{1}}{\psi_{2}}$ for some $\psi_{i} \in H^{p}$ $(p>0, i=1,2)$. If $\psi_{2}=\psi^{i} \psi^{e}$ is the inner-outer factorization of $\psi_{2}$, then $\phi=\overline{\psi^{i}} \frac{\psi_{1}}{\psi^{e}}$. Thus if $\phi \in L^{2}$ is of bounded type, then $\phi$ can be written as

$$
\phi=\bar{\theta} a
$$

where $\theta$ is inner, $a \in H^{2}$ and $\theta$ and $a$ are coprime.

Write $\mathbb{D}^{e}:=\{z: 1<|z| \leq \infty\}$. For a function $g: \mathbb{D}^{e} \rightarrow \mathbb{C}$, define a function $g_{\mathbb{D}}: \mathbb{D} \rightarrow \mathbb{C}$ by

$$
g_{\mathbb{D}}(\zeta):=\overline{g(1 / \bar{\zeta})} \quad(\zeta \in \mathbb{D}) .
$$

For a function $g: \mathbb{D}^{e} \rightarrow \mathbb{C}$, we say that $g$ belongs to $H^{p}\left(\mathbb{D}^{e}\right)$ if $g_{\mathbb{D}} \in H^{p}(1 \leq p \leq \infty)$. A function $g: \mathbb{D}^{e} \rightarrow \mathbb{C}$ is said to be of bounded type if $g_{\mathbb{D}}$ is of bounded type. If $f \in H^{2}$, then the function $\hat{f}$ defined in $\mathbb{D}^{e}$ is called a pseudo-continuation of $f$ if $\hat{f}$ is a function of bounded type and $\hat{f}(z)=f(z)$ for almost all $z \in \mathbb{T}$ (cf. $\mathbf{B B}$, Ni1, Sh]). Then we can easily show that $\breve{f}$ is of bounded type if and only if $f$ has a pseudo-continuation $\hat{f}$. In this case, $\hat{f}_{\mathbb{D}}(z)=\overline{f(z)}$ for almost all $z \in \mathbb{T}$. In particular,

(2.1) $\phi \equiv \breve{\phi}_{-}+\phi_{+} \in L^{2}$ is of bounded type $\Longleftrightarrow \phi_{-}$has a pseudo-continuation.

We review here a few essential facts concerning vector-valued $L^{p}$ and $H^{p_{-}}$ functions that we will used to begin with, using $[\mathbf{D S}], \mathbf{D u},[\mathbf{F F},[\mathbf{H P},[\mathbf{H o}$, Ni1, $\mathbf{N i 2}, \mathbf{P e}, \mathbf{S a}$ as general references.

Let $(\Omega, \mathfrak{M}, \mu)$ be a positive $\sigma$-finite measure space and $X$ be a complex Banach space. A function $f: \Omega \rightarrow X$ of the form $f=\sum_{k=1}^{\infty} x_{k} \chi_{\sigma_{k}}$ (where $x_{k} \in X, \sigma_{k} \in \mathfrak{M}$ and $\sigma_{k} \cap \sigma_{j}=\emptyset$ for $k \neq j$ ) is said to be countable-valued. A function $f: \Omega \rightarrow X$ is called weakly measurable if the map $s \mapsto \phi(f(s))$ is measurable for all $\phi \in X^{*}$ and is called strongly measurable if there exist countable-valued functions $f_{n}$ such that $f(s)=\lim _{n} f_{n}(s)$ for almost all $s \in \Omega$. It is known that when $X$ is separable,

(i) if $f$ is weakly measurable, then $\|f(\cdot)\|$ is measurable;

(ii) $f$ is strongly measurable if and only if it is weakly measurable.

A countable-valued function $f=\sum_{k=1}^{\infty} x_{k} \chi_{\sigma_{k}}$ is called (Bochner) integrable if

$$
\int_{\Omega}\|f(s)\| d \mu(s)<\infty
$$

and its integral is defined by

$$
\int_{\Omega} f d \mu:=\sum_{k=1}^{\infty} x_{k} \mu\left(\sigma_{k}\right)
$$

A function $g: \Omega \rightarrow X$ is called integrable if there exist countable-valued integrable functions $g_{n}$ such that $g(s)=\lim _{n} g_{n}(s)$ for almost all $s \in \Omega$ and $\lim _{n} \int_{\Omega} \| g-$ $g_{n} \| d \mu=0$. Then $\int_{\Omega} g d \mu \equiv \lim _{n} \int_{\Omega} g_{n} d \mu$ exists and $\int_{\Omega} g d \mu$ is called the (Bochner) integral of $g$. If $f: \Omega \rightarrow X$ is integrable, then we can see that

$$
T\left(\int_{\Omega} f d \mu\right)=\int_{\Omega}(T f) d \mu \quad \text { for each } T \in \mathcal{B}(X, Y) .
$$


Let $m$ denote the normalized Lebesgue measure on $\mathbb{T}$. For a complex Banach space $X$ and $1 \leq p \leq \infty$, let

$$
L_{X}^{p} \equiv L^{p}(\mathbb{T}, X):=\left\{f: \mathbb{T} \rightarrow X: f \text { is strongly measurable and }\|f\|_{p}<\infty\right\},
$$

where

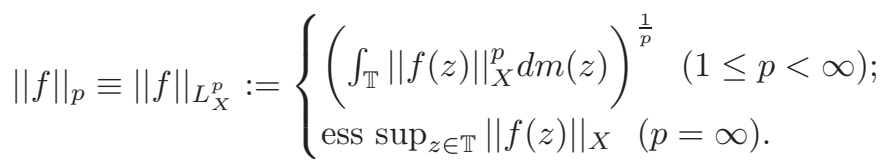

Then we can see that $L_{X}^{p}$ forms a Banach space. For $f \in L_{X}^{1}$, the $n$-th Fourier coefficient of $f$, denoted by $\widehat{f}(n)$, is defined by

$$
\widehat{f}(n):=\int_{\mathbb{T}} \bar{z}^{n} f(z) d m(z) \text { for each } n \in \mathbb{Z} .
$$

Also, $H_{X}^{p} \equiv H^{p}(\mathbb{T}, X)$ is defined by the set of $f \in L_{X}^{p}$ with $\widehat{f}(n)=0$ for $n<0$. A function $f: \mathbb{D} \rightarrow X$ is (norm) analytic if $f$ can be written as

$$
f(\zeta)=\sum_{n=0}^{\infty} x_{n} \zeta^{n} \quad\left(\zeta \in \mathbb{D}, x_{n} \in X\right),
$$

Let $\operatorname{Hol}(\mathbb{D}, X)$ denote the set of all analytic functions $f: \mathbb{D} \rightarrow X$. Also we write $H^{2}(\mathbb{D}, X)$ for the set of all $f \in \operatorname{Hol}(\mathbb{D}, X)$ satisfying

$$
\|f\|_{H^{2}(\mathbb{D}, X)}:=\sup _{0<r<1}\left(\int_{\mathbb{T}}\|f(r z)\|_{X}^{2} d m(z)\right)^{\frac{1}{2}}<\infty .
$$

Let $E$ be a separable complex Hilbert space. As in the scalar-valued case, if $f \in H^{2}(\mathbb{D}, E)$, then there exists a "boundary function" bf $\in H_{E}^{2}$ such that

$$
f(r z)=\left(b f * P_{r}\right)(z) \quad(r \in[0,1) \text { and } z \in \mathbb{T})
$$

(where $P_{r}$ denotes the Poisson kernel) and

$$
(b f)(z)=\lim _{r z \rightarrow z} f(r z) \text { nontangentially a.e. on } \mathbb{T} \text {. }
$$

Moreover, the mapping $f \mapsto b f$ is an isometric bijection (cf. [Ni2, Theorem 3.11.7]). We conventionally identify $H^{2}(\mathbb{D}, E)$ with $H_{E}^{2} \equiv H^{2}(\mathbb{T}, E)$. For $f, g \in L_{E}^{2}$ with a separable complex Hilbert space $E$, the inner product $\langle f, g\rangle$ is defined by

$$
\langle f, g\rangle \equiv\langle f(z), g(z)\rangle_{L_{E}^{2}}:=\int_{\mathbb{T}}\langle f(z), g(z)\rangle_{E} d m(z) .
$$

If $f, g \in L_{X}^{2}$ with $X=M_{n \times m}$, then $\langle f, g\rangle=\int_{\mathbb{T}} \operatorname{tr}\left(g^{*} f\right) d m$.

For a function $\Phi: \mathbb{T} \rightarrow \mathcal{B}(D, E)$, write

$$
\Phi^{*}(z):=\Phi(z)^{*} \quad \text { for } z \in \mathbb{T} .
$$

A function $\Phi: \mathbb{T} \rightarrow \mathcal{B}(X, Y)$ is called SOT measurable if $z \mapsto \Phi(z) x$ is strongly measurable for every $x \in X$ and is called WOT measurable if $z \mapsto \Phi(z) x$ is weakly measurable for every $x \in X$. We can easily check that if $\Phi: \mathbb{T} \rightarrow \mathcal{B}(X, Y)$ is strongly measurable, then $\Phi$ is SOT-measurable and if $D$ and $E$ are separable complex Hilbert spaces then $\Phi: \mathbb{T} \rightarrow \mathcal{B}(D, E)$ is SOT measurable if and only if $\Phi$ is WOT measurable.

We then have:

Lemma 2.1. If $\Phi: \mathbb{T} \rightarrow \mathcal{B}(D, E)$ is WOT measurable, then so is $\Phi^{*}$. 
Proof. Suppose that $\Phi$ is WOT measurable. Then the function

$$
z \mapsto \overline{\left\langle\Phi^{*}(z) y, x\right\rangle}=\left\langle x, \Phi^{*}(z) y\right\rangle=\langle\Phi(z) x, y\rangle
$$

is measurable for all $x \in D$ and $y \in E$. Thus the function $z \mapsto\left\langle\Phi^{*}(z) y, x\right\rangle$ is measurable for all $x \in D$ and $y \in E$.

Let $\Phi: \mathbb{T} \rightarrow \mathcal{B}(D, E)$ be a WOT measurable function. Then $\Phi$ is called WOT integrable if $\langle\Phi(\cdot) x, y\rangle \in L^{1}$ for every $x \in D$ and $y \in E$, and there exists an operator $U \in \mathcal{B}(D, E)$ such that $\langle U x, y\rangle=\int_{\mathbb{T}}\langle\Phi(z) x, y\rangle d m(z)$. Also $\Phi$ is called SOT integrable if $\Phi(\cdot) x$ is integrable for every $x \in D$. In this case, the operator $V: x \mapsto \int_{\mathbb{T}} \Phi(z) x d m(z)$ is bounded, i.e., $V \in \mathcal{B}(D, E)$. If $\Phi: \mathbb{T} \rightarrow \mathcal{B}(D, E)$ is SOT integrable, then it follows from (2.2) that for every $x \in D$ and $y \in E$,

$$
\left\langle\int_{\mathbb{T}} \Phi(z) x d m(z), y\right\rangle=\int_{\mathbb{T}}\langle\Phi(z) x, y\rangle d m(z),
$$

which implies that $\Phi$ is WOT integrable and that the SOT integral of $\Phi$ is equal to the WOT integral of $\Phi$.

We can say more:

Lemma 2.2. For $\Phi \in L_{\mathcal{B}(D, E)}^{1}$, the Bochner integral of $\Phi$ is equal to the SOT integral of $\Phi$, in the sense that

$$
\left(\int_{\mathbb{T}} \Phi(z) d m(z)\right) x=\int_{\mathbb{T}} \Phi(z) x d m(z) \quad \text { for all } x \in D .
$$

Proof. This follows from a straightforward calculation. 


\section{CHAPTER 3}

\section{Strong $L^{2}$-functions}

To examine Question 1.1 we need to consider operator-valued functions defined on the unit circle constructed by arranging the vectors in $F$ as their column vectors. Using this viewpoint, we will consider operator-valued functions whose "column" vectors are $L^{2}$-functions. Note that (bounded linear) operators between separable Hilbert spaces may be represented as infinite matrices, so that column vectors of operators are well justified. This viewpoint leads us to define (operator-valued) strong $L^{2}$-functions. In this chapter we consider strong $L^{2}$-functions and then derive some of their properties.

The terminology of a "strong $H^{2}$-function" is reserved for the operator-valued functions on the unit disk $\mathbb{D}$, following to N.K. Nikolskii [Ni1: A function $\Phi: \mathbb{D} \rightarrow$ $\mathcal{B}(D, E)$ is called a strong $H^{2}$-function if $\Phi(\cdot) x \in H^{2}(\mathbb{D}, E)$ for each $x \in D$. To describe this in detail, and to explain the crucial role that strong $L^{2}$-functions play in our theory, we need to introduce some additional notation and terminology.

Let $L^{\infty}(\mathcal{B}(D, E))$ be the space of all bounded (WOT) measurable $\mathcal{B}(D, E)$ valued functions on $\mathbb{T}$. For $\Psi \in L^{\infty}(\mathcal{B}(D, E))$, define

$$
\|\Psi\|_{\infty}:=\operatorname{ess}_{\sup } z \in \mathbb{T}\|\Psi(z)\| \text {. }
$$

For $1 \leq p<\infty$, we define the class $L_{s}^{p}(\mathcal{B}(D, E)) \equiv L_{s}^{p}(\mathbb{T}, \mathcal{B}(D, E))$ as the set of all (WOT) measurable $\mathcal{B}(D, E)$-valued functions $\Phi$ on $\mathbb{T}$ such that $\Phi(\cdot) x \in L_{E}^{p}$. A function $\Phi \in L_{s}^{p}(\mathcal{B}(D, E))$ is called a strong $L^{p}$-function. We claim that

$$
L_{\mathcal{B}(D, E)}^{p} \subseteq L_{s}^{p}(\mathcal{B}(D, E)):
$$

indeed if $\Phi \in L_{\mathcal{B}(D, E)}^{p}$, then for all $x \in D$ with $\|x\|=1$,

$$
\|\Phi(z) x\|_{L_{E}^{p}}^{p}=\int_{\mathbb{T}}\|\Phi(z) x\|_{E}^{p} d m(z) \leq \int_{\mathbb{T}}\|\Phi(z)\|_{\mathcal{B}(D, E)}^{p} d m(z)=\|\Phi\|_{L_{\mathcal{B}(D, E)}^{p}}^{p},
$$

which gives (3.1). Also we can easily check that

$$
L_{\mathcal{B}(D, E)}^{\infty} \subseteq L^{\infty}(\mathcal{B}(D, E)) \subseteq L_{s}^{p}(\mathcal{B}(D, E)) .
$$

Remark 3.1. We may define a norm on $L_{s}^{p}(\mathcal{B}(D, E))$ : i.e.,

$$
\|\Phi\|_{p}^{(s)}:=\sup \left\{\|\Phi(z) x\|_{L_{E}^{p}}: x \in D \text { with }\|x\|=1\right\} .
$$

Then $L_{s}^{p}(\mathcal{B}(D, E))$ forms a normed space for $1 \leq p<\infty$. Moreover, we can show that $\|\Phi\|_{p}^{(s)}$ is a complete norm for $1 \leq p<\infty$, i.e., $L_{s}^{p}(\mathcal{B}(D, E))$ is a Banach 
space for $1 \leq p<\infty$. However, in general, we cannot guarantee that $\|\Phi\|_{p}^{(s)}=$ $\|\Phi\|_{L_{\mathcal{B}(D, E)}^{p}}$. To see this, let $C$ be the upper unit circle and $1 \leq p<\infty$. Put

$$
\Phi:=\left[\begin{array}{cc}
\chi_{C} & 0 \\
0 & 1-\chi_{C}
\end{array}\right] .
$$

Then $\|\Phi(z)\|=1$ for all $z \in \mathbb{T}$, so that $\|\Phi\|_{L_{M_{2}}^{p}}=1$. Let $x:=[\alpha, \beta]^{t}$ be a unit vector in $\mathbb{C}^{2}$. Then we have that

$$
\|\Phi(z) x\|_{L_{C^{2}}^{p}}^{p}=\int_{\mathbb{T}}\left\|\left[\alpha \chi_{C}, \beta\left(1-\chi_{C}\right)\right]^{t}\right\|^{p} d m(z)=\frac{1}{2}\left(|\alpha|^{p}+|\beta|^{p}\right) \leq \frac{1}{\sqrt{2}},
$$

which gives $\|\Phi\|_{p}^{(s)} \neq\|\Phi\|_{L_{M_{2}}^{p}}$.

If $\Phi \in L_{s}^{1}(\mathcal{B}(D, E))$ and $x \in D$, then $\Phi(\cdot) x \in L_{E}^{1}$. Thus the $n$-th Fourier coefficient $\widehat{\Phi(\cdot) x}(n)$ of $\Phi(\cdot) x$ is given by

$$
\widehat{\Phi(\cdot) x}(n)=\int_{\mathbb{T}} \bar{z}^{n} \Phi(z) x d m(z)
$$

We now define the $n$-th Fourier coefficient of $\Phi \in L_{s}^{1}(\mathcal{B}(D, E))$, denoted by $\widehat{\Phi}(n)$, by

$$
\widehat{\Phi}(n) x:=\widehat{\Phi(\cdot) x}(n) \quad(n \in \mathbb{Z}, x \in D) .
$$

We define

$$
H_{s}^{2}(\mathcal{B}(D, E)) \equiv H_{s}^{2}(\mathbb{T}, \mathcal{B}(D, E)):=\left\{\Phi \in L_{s}^{2}(\mathcal{B}(D, E)): \widehat{\Phi}(n)=0 \text { for } n<0\right\},
$$

or equivalently, $H_{s}^{2}(\mathcal{B}(D, E))$ is the set of all WOT measurable functions $\Phi$ on $\mathbb{T}$ such that $\Phi(\cdot) x \in H_{E}^{2}$ for each $x \in D$. We also define

$$
H^{\infty}(\mathcal{B}(D, E)) \equiv H^{\infty}(\mathbb{T}, \mathcal{B}(D, E)):=\left\{\Phi \in L^{\infty}(\mathcal{B}(D, E)): \widehat{\Phi}(n)=0 \text { for } n<0\right\} .
$$

On the other hand, we define $H^{\infty}(\mathbb{D}, \mathcal{B}(D, E))$ as the set of all analytic functions $\Phi: \mathbb{D} \rightarrow \mathcal{B}(D, E)$ satisfying

$$
\|\Phi\|_{H^{\infty}}:=\sup _{\zeta \in \mathbb{D}}\|\Phi(\zeta)\| .
$$

If $D$ and $E$ are separable Hilbert spaces, we conventionally identify $H^{\infty}(\mathbb{D}, \mathcal{B}(D, E))$ with $H^{\infty}(\mathbb{T}, \mathcal{B}(D, E))$ (cf. [Ni2, Theorem 3.11.10]).

On the other hand, by (3.1), we have $L_{\mathcal{B}(D, E)}^{1} \subseteq L_{s}^{1}(\mathcal{B}(D, E))$. Thus if $\Phi \in L_{\mathcal{B}(D, E)}^{1}$, then there are two definitions of the $n$-th Fourier coefficient of $\Phi$. However, we can, by Lemma 2.2. see that the $n$-th Fourier coefficient of $\Phi$ as an element of $L_{\mathcal{B}(D, E)}^{1}$ coincides with the $n$-th Fourier coefficient of $\Phi$ as an element of $L_{s}^{1}(\mathcal{B}(D, E))$.

We now denote by $H_{s}^{2}(\mathbb{D}, \mathcal{B}(D, E))$ the set of all strong $H^{2}$-functions with values in $\mathcal{B}(D, E)$.

We then have:

Lemma 3.2. $H^{2}(\mathbb{D}, \mathcal{B}(D, E)) \subseteq H_{s}^{2}(\mathbb{D}, \mathcal{B}(D, E))$. 
Proof. Let $\Phi \in H^{2}(\mathbb{D}, \mathcal{B}(D, E))$. Then $\Phi$ can be written as

$$
\Phi(\zeta)=\sum_{n=0}^{\infty} A_{n} \zeta^{n} \quad\left(A_{n} \in \mathcal{B}(D, E)\right)
$$

Thus for each $x \in D$,

$$
\Phi(\zeta) x=\sum_{n=0}^{\infty}\left(A_{n} x\right) \zeta^{n} \in \operatorname{Hol}(\mathbb{D}, E)
$$

Observe that

$$
\begin{aligned}
\|\Phi(\cdot) x\|_{H^{2}(\mathbb{D}, E)}^{2} & =\sup _{0<r<1} \int_{\mathbb{T}}\|\Phi(r z) x\|_{E}^{2} d m(z) \\
& \leq\|\Phi\|_{H^{2}(\mathbb{D}, \mathcal{B}(D, E))}^{2} \cdot\|x\|_{D}^{2} \\
& <\infty
\end{aligned}
$$

which implies $\Phi \in H_{s}^{2}(\mathbb{D}, \mathcal{B}(D, E))$.

THEOREM 3.3. If $\operatorname{dim} D<\infty$, then

$$
H^{2}(\mathbb{D}, \mathcal{B}(D, E))=H_{s}^{2}(\mathbb{D}, \mathcal{B}(D, E)),
$$

where the equality is set-theoretic.

Proof. By Lemma 3.2 , we have $H^{2}(\mathbb{D}, \mathcal{B}(D, E)) \subseteq H_{s}^{2}(\mathbb{D}, \mathcal{B}(D, E))$. For the reverse inclusion, suppose $\Phi \in H_{s}^{2}(\mathbb{D}, \mathcal{B}(D, E))$ and $\operatorname{dim} D=d<\infty$. Let $\left\{e_{j}: j=1,2, \cdots, d\right\}$ be an orthonormal basis of $D$. Then for each $j=1,2, \cdots, d$,

$$
\phi_{j}(\zeta) \equiv \Phi(\zeta) e_{j} \in H^{2}(\mathbb{D}, E)
$$

Thus we may write

$$
\phi_{j}(\zeta)=\sum_{n=0}^{\infty} a_{n}^{(j)} \zeta^{n} \quad\left(a_{n}^{(j)} \in E\right) .
$$

For each $n=0,1,2, \cdots$, define $A_{n}: D \rightarrow E$ by

$$
A_{n} x:=\sum_{j=1}^{d} \alpha_{j} a_{n}^{(j)} \quad\left(\text { where } x:=\sum_{j=1}^{d} \alpha_{j} e_{j}\right) .
$$

Then $A_{n} \in \mathcal{B}(D, E)$. We claim that

$$
\Phi(\zeta)=\sum_{n=0}^{\infty} A_{n} \zeta^{n} \in \operatorname{Hol}(\mathbb{D}, \mathcal{B}(D, E)) .
$$

To prove (3.4), let $\epsilon>0$ be arbitrary. For each $\zeta \in \mathbb{D}$, there exists $M>0$ such that for all $j=1,2, \cdots, d$,

$$
\left\|\sum_{n=M}^{\infty} a_{n}^{(j)} \zeta^{n}\right\|_{E}<\frac{\epsilon}{d}
$$


Let $x:=\sum_{j=1}^{d} \alpha_{j} e_{j}$ with $\|x\|_{D}=1$. Then we have

$$
\begin{aligned}
\left\|\left(\Phi(\zeta)-\sum_{n=0}^{M-1} A_{n} \zeta^{n}\right) x\right\|_{E} & =\left\|\sum_{n=M}^{\infty} \sum_{j=1}^{d} \alpha_{j} a_{n}^{(j)} \zeta^{n}\right\|_{E} \\
& \leq \sum_{j=1}^{d}\left\|\sum_{n=M}^{\infty} a_{n}^{(j)} \zeta^{n}\right\|_{E} \\
& <\epsilon,
\end{aligned}
$$

which proves (3.4). For all $r \in[0,1)$, we have that

$$
\begin{aligned}
\|\Phi(r z) x\|_{E}^{2} & =\left\|\sum_{j=1}^{d} \alpha_{j} \Phi(r z) e_{j}\right\|_{E}^{2} \\
& \leq\left(\sum_{j=1}^{d}\left|\alpha_{j}\left\|\mid \Phi(r z) e_{j}\right\|_{E}\right)^{2}\right. \\
& \leq \sum_{j=1}^{d}\left\|\Phi(r z) e_{j}\right\|_{E}^{2} .
\end{aligned}
$$

Thus $\|\Phi(r z)\|_{\mathcal{B}(D, E)}^{2} \leq \sum_{j=1}^{d}\left\|\Phi(r z) e_{j}\right\|_{E}^{2}$, and hence it follows from (3.3) that

$$
\begin{aligned}
\|\Phi\|_{H^{2}(\mathbb{D}, \mathcal{B}(D, E))} & =\sup _{0<r<1} \int_{\mathbb{T}}\|\Phi(r z)\|_{\mathcal{B}(D, E)}^{2} d m(z) \\
& \leq \sup _{0<r<1} \int_{\mathbb{T}} \sum_{j=1}^{d}\left\|\Phi(r z) e_{j}\right\|_{E}^{2} d m(z) \\
& \leq \sum_{j=1}^{d}\left\|\phi_{j}\right\|_{H^{2}(\mathbb{D}, E)}^{2}<\infty
\end{aligned}
$$

which implies $\Phi \in H^{2}(\mathbb{D}, \mathcal{B}(D, E))$. This completes the proof.

Remark 3.4. Theorem 3.3 may fail if the condition " $\operatorname{dim} D<\infty$ is dropped. For example, if $\Phi$ is defined on the unit disk $\mathbb{D}$ by

$$
\Phi(\zeta):=\left[\begin{array}{llll}
\zeta & \zeta^{2} & \zeta^{3} & \cdots
\end{array}\right]: \ell^{2} \rightarrow \mathbb{C} \quad(\zeta \in \mathbb{D}),
$$

then $\Phi(\zeta)$ is a bounded linear operator for each $\zeta \in \mathbb{D}$ : indeed,

$$
\begin{aligned}
\|\Phi(\zeta)\|_{\mathcal{B}\left(\ell^{2}, \mathbb{C}\right)} & =\sup _{\|x\|=1}|\Phi(\zeta) x| \\
& =\sup _{\|x\|=1}\left|\sum_{n=1}^{\infty} \zeta^{n} x_{n}\right| \quad\left(x \equiv\left(x_{n}\right) \in \ell^{2}\right) \\
& =\sup _{\|x\|=1}\left|\left\langle\left(\zeta, \zeta^{2}, \zeta^{3}, \cdots\right),\left(\bar{x}_{1}, \bar{x}_{2}, \bar{x}_{3}, \cdots\right)\right\rangle\right| \\
& =\left\|\left(\zeta, \zeta^{2}, \zeta^{3}, \cdots\right)\right\|_{\ell^{2}} \\
& =\left(\frac{|\zeta|^{2}}{1-|\zeta|^{2}}\right)^{\frac{1}{2}} .
\end{aligned}
$$


Moreover, for each $x \equiv\left(x_{n}\right) \in \ell^{2}$,

$$
\Phi(\zeta) x=\sum_{n=1}^{\infty} x_{n} \zeta^{n} \in H^{2}(\mathbb{D}, \mathbb{C}),
$$

which says that $\Phi \in H_{s}^{2}\left(\mathbb{D}, \mathcal{B}\left(\ell^{2}, \mathbb{C}\right)\right)$. However, we have $\Phi \notin H^{2}\left(\mathbb{D}, \mathcal{B}\left(\ell^{2}, \mathbb{C}\right)\right)$ : indeed, for $\zeta=r z \in \mathbb{D}$,

$$
\|\Phi(\zeta)\|_{\mathcal{B}\left(\ell^{2}, \mathbb{C}\right)}^{2}=\left\|\Phi(\zeta) \Phi(\zeta)^{*}\right\|_{\mathcal{B}\left(\ell^{2}, \mathbb{C}\right)}=\frac{r^{2}}{1-r^{2}},
$$

so that

$$
\begin{aligned}
\sup _{0<r<1} \int_{\mathbb{T}}\|\Phi(r z)\|_{\mathcal{B}\left(\ell^{2}, \mathbb{C}\right)}^{2} d m(z) & =\sup _{0<r<1} \int_{\mathbb{T}} \frac{r^{2}}{1-r^{2}} d m(z) \\
& =\sup _{0<r<1} \frac{r^{2}}{1-r^{2}} \\
& =\infty .
\end{aligned}
$$

In general, the boundary values of strong $\mathrm{H}^{2}$-functions do not need to be bounded linear operators (defined almost everywhere on $\mathbb{T}$ ). Thus we do not guarantee that the boundary value of a strong $H^{2}$-function belongs to $H_{s}^{2}(\mathbb{T}, \mathcal{B}(D, E))$. For example, if $\Phi$ is defined on the unit disk $\mathbb{D}$ by

$$
\Phi(\zeta)=\left[\begin{array}{lllll}
1 & \zeta & \zeta^{2} & \zeta^{3} & \ldots
\end{array}\right]: \ell^{2} \rightarrow \mathbb{C} \quad(\zeta \in \mathbb{D}),
$$

then by Remark 3.4, $\Phi$ is a strong $H^{2}$-function with values in $\mathcal{B}\left(\ell^{2}, \mathbb{C}\right)$. However, the boundary value

$$
\Phi(z)=\left[\begin{array}{lllll}
1 & z & z^{2} & z^{3} & \cdots
\end{array}\right]: \ell^{2} \rightarrow \mathbb{C} \quad(z \in \mathbb{T})
$$

is not bounded for all $z \in \mathbb{T}$ because for any $z_{0} \in \mathbb{T}$, if we let

$$
x_{0}:=\left(1, \bar{z}_{0}, \frac{\bar{z}_{0}^{2}}{2}, \frac{\bar{z}_{0}^{3}}{3}, \cdots\right)^{t} \in \ell^{2},
$$

then

$$
\Phi\left(z_{0}\right) x_{0}=1+\sum_{n=1}^{\infty} \frac{1}{n}=\infty,
$$

which shows that $\Phi \notin H_{s}^{2}(\mathbb{T}, \mathcal{B}(D, E))$.

In spite of it, there are useful relations between the set $H_{s}^{2}(\mathbb{D}, \mathcal{B}(D, E))$ and the set $H_{s}^{2}(\mathbb{T}, \mathcal{B}(D, E))$. To see this, let $\Phi \in H_{s}^{2}(\mathbb{T}, \mathcal{B}(D, E))$. Then $\Phi(z) \in \mathcal{B}(D, E)$ for almost all $z \in \mathbb{T}$ and $\Phi(z) x \in H_{E}^{2}$ for each $x \in D$. We now define a (functionvalued with domain $D$ ) function $p \Phi$ on the unit disk $\mathbb{D}$ by the Poisson integral in the strong sense:

$$
\begin{aligned}
p \Phi\left(r e^{i \theta}\right) x & :=\left(\Phi(\cdot) x * P_{r}\right)\left(e^{i \theta}\right) \quad(x \in D) \\
& =\int_{0}^{2 \pi} P_{r}(\theta-t) \Phi\left(e^{i t}\right) x d m(t) \in E,
\end{aligned}
$$

where $P_{r}(\cdot)$ is the Poisson kernel. Then $p \Phi(\zeta) x \in H^{2}(\mathbb{D}, E)$. Thus, for all $\zeta \in \mathbb{D}$, $p \Phi(\zeta)$ can be viewed as a function from $D$ into $E$. A straightforward calculation shows that $p \Phi(\zeta)$ is a linear map for each $\zeta \in \mathbb{D}$. Since $p \Phi(\zeta) x \in H^{2}(\mathbb{D}, E)$ is the Poisson integral of $\Phi(z) x \in H_{E}^{2}$, we will conventionally identify $\Phi(z) x$ and $p \Phi(\zeta) x$ 
for each $x \in D$. From this viewpoint, we will also regard $\Phi \in H_{s}^{2}(\mathbb{T}, \mathcal{B}(D, E))$ as an (linear, but not necessarily bounded) operator-valued function defined on the unit disk $\mathbb{D}$.

We thus have:

LEMma 3.5. The following inclusion holds:

$$
H_{\mathcal{B}(D, E)}^{2} \cup H^{\infty}(\mathcal{B}(D, E)) \subseteq H_{s}^{2}(\mathbb{D}, \mathcal{B}(D, E)) .
$$

Proof. Note that by (3.1) and (3.2), $H_{\mathcal{B}(D, E)}^{2} \cup H^{\infty}(\mathcal{B}(D, E)) \subseteq H_{s}^{2}(\mathbb{T}, \mathcal{B}(D, E))$. Thus in view of the preceding remark, it suffices to show $\Phi(\zeta) \in \mathcal{B}(D, E)$ for all $\zeta \in \mathbb{D}$. To see this we first claim that there exists $M>0$ such that

$$
\sup \left\{\|\Phi(\cdot) x\|_{L_{E}^{1}}: x \in D \text { with }\|x\|=1\right\}<M,
$$

To see this, if $\Phi \in H_{\mathcal{B}(D, E)}^{2}$, then for all $x \in D$ with $\|x\|=1$,

$$
\begin{aligned}
\|\Phi(\cdot) x\|_{L_{E}^{1}} & \leq\|\Phi(\cdot) x\|_{L_{E}^{2}} \\
& \leq\left(\int_{\mathbb{T}}\|\Phi(z)\|_{\mathcal{B}(D, E)}^{2} d m(z)\right)^{\frac{1}{2}} \\
& =\|\Phi\|_{L_{\mathcal{B}(D, E)}^{2}} .
\end{aligned}
$$

If instead $\Phi \in H^{\infty}(\mathcal{B}(D, E))$, then for all $x \in D$ with $\|x\|=1$,

$$
\|\Phi(\cdot) x\|_{L_{E}^{1}}=\int_{\mathbb{T}}\|\Phi(z) x\|_{E} d m(z) \leq\|\Phi(z)\|_{\infty}
$$

which proves the claim (3.5). Now, let $\zeta=r e^{i \theta} \in \mathbb{D}$ and $x \in D$ with $\|x\|=1$. Then for $y \in E$ with $\|y\| \leq 1$,

$$
\begin{aligned}
\left|\left\langle\Phi\left(r e^{i \theta}\right) x, y\right\rangle_{E}\right| & =\left|\left\langle\int_{0}^{2 \pi} P_{r}(\theta-t) \Phi\left(e^{i t}\right) x d m(t), y\right\rangle_{E}\right| \\
& =\left|\int_{0}^{2 \pi}\left\langle P_{r}(\theta-t) \Phi\left(e^{i t}\right) x, y\right\rangle_{E} d m(t)\right| \quad \text { (by (2.3) ) } \\
& \leq \frac{1+r}{1-r} \int_{0}^{2 \pi}\left|\left\langle\Phi\left(e^{i t}\right) x, y\right\rangle_{E}\right| d m(t)
\end{aligned}
$$

which implies, by our assumption,

$$
\begin{aligned}
\|\Phi(\zeta) x\|_{E} & \leq \frac{1+r}{1-r} \int_{0}^{2 \pi}\left\|\Phi\left(e^{i t}\right) x\right\|_{E} d m(t) \\
& =\frac{1+r}{1-r}\|\Phi(\cdot) x\|_{L_{E}^{1}} \\
& <\infty
\end{aligned}
$$

which shows that $\Phi(\zeta) \in \mathcal{B}(D, E)$ for all $\zeta \in \mathbb{D}$. Thus we have $\Phi \in H_{s}^{2}(\mathbb{D}, \mathcal{B}(D, E))$.

We now recall a notion from classical Banach space theory, about regarding a vector as an operator acting on the scalars. This notion is important as motivation 
for the study of strong $L^{2}$-functions. Let $E$ be a separable complex Hilbert space. For a function $f: \mathbb{T} \rightarrow E$, define $[f]: \mathbb{T} \rightarrow \mathcal{B}(\mathbb{C}, E)$ by

$$
[f](z) \alpha:=\alpha f(z) \quad(\alpha \in \mathbb{C}) .
$$

If $g: \mathbb{T} \rightarrow E$ is a countable-valued function of the form

$$
g=\sum_{k=1}^{\infty} x_{k} \chi_{\sigma_{k}} \quad\left(x_{k} \in E\right)
$$

then for each $\alpha \in \mathbb{C}$,

$$
\left(\sum_{k=1}^{\infty}\left[x_{k}\right] \chi_{\sigma_{k}}\right) \alpha=\sum_{k=1}^{\infty} \alpha x_{k} \chi_{\sigma_{k}}=\alpha g=[g] \alpha,
$$

which implies that $[g]$ is a countable-valued function of the form $[g]=\sum_{k=1}^{\infty}\left[x_{k}\right] \chi_{\sigma_{k}}$.

We then have:

Lemma 3.6. Let $E$ be a separable complex Hilbert space and $1 \leq p \leq \infty$. Define $\Gamma: L_{E}^{p} \rightarrow L_{\mathcal{B}(\mathbb{C}, E)}^{p}$ by

$$
\Gamma(f)(z)=[f](z),
$$

where $[f](z): \mathbb{C} \rightarrow E$ is given by $[f](z) \alpha:=\alpha f(z)$. Then

(a) $\Gamma$ is unitary, and hence $L_{E}^{p} \cong L_{\mathcal{B}(\mathbb{C}, E)}^{p}$;

(b) $L_{\mathcal{B}(\mathbb{C}, E)}^{p}=L_{s}^{p}(\mathcal{B}(\mathbb{C}, E))$ for $1 \leq p<\infty$;

(c) $\widehat{[f]}(n)=[\widehat{f}(n)]$ for $f \in L_{E}^{p}$ and $n \in \mathbb{Z}$.

In particular, $H_{E}^{p} \cong H_{\mathcal{B}(\mathbb{C}, E)}^{p}=H_{s}^{p}(\mathcal{B}(\mathbb{C}, E))$ for $1 \leq p<\infty$.

Proof. (a) Let $f \in L_{E}^{p}(1 \leq p \leq \infty)$ be arbitrary. We first show that $[f] \in$ $L_{\mathcal{B}(\mathbb{C}, E)}^{p}$. Since $f$ is strongly measurable, there exist countable-valued functions $f_{n}$ such that $f(z)=\lim _{n} f_{n}(z)$ for almost all $z \in \mathbb{T}$. Observe that for almost all $z \in \mathbb{T}$,

Thus we have that

$$
\|[f](z)\|_{\mathcal{B}(\mathbb{C}, E)}=\sup _{|\alpha|=1}||[f](z) \alpha\left\|_{E}=\right\| f(z) \|_{E} .
$$

$$
\left\|\left[f_{n}\right](z)-[f](z)\right\|_{\mathcal{B}(\mathbb{C}, E)}=\left\|f_{n}(z)-f(z)\right\|_{E} \rightarrow 0 \quad \text { as } n \rightarrow \infty,
$$

which implies that $[f]$ is strongly measurable and $\|[f]\|_{L_{\mathcal{B}(\mathbb{C}, E)}^{p}}=\|f\|_{L_{E}^{p}}$. Thus $\Gamma$ is an isometry. For $h \in L_{\mathcal{B}(\mathbb{C}, E)}^{p}$, let $g(z):=h(z) 1 \in L_{E}^{p}$. Then for all $\alpha \in \mathbb{C}$, we have

$$
\Gamma(g)(z) \alpha=\alpha h(z) 1=h(z) \alpha,
$$

which implies that $\Gamma$ is a surjection from $L_{E}^{p}$ onto $L_{\mathcal{B}(\mathbb{C}, E)}^{p}$. Thus $\Gamma$ is unitary, so that $L_{E}^{p} \cong L_{\mathcal{B}(\mathbb{C}, E)}^{p}$. This proves (a).

(b) Suppose $h \in L_{s}^{p}(\mathcal{B}(\mathbb{C}, E))(1 \leq p<\infty)$. If $g(z):=h(z) 1 \in L_{E}^{p}$, then $h=[g] \in L_{\mathcal{B}(\mathbb{C}, E)}^{p}$. The converse is clear.

(c) Let $f \in L_{E}^{p}$. Then for all $\alpha \in \mathbb{C}$ and $n \in \mathbb{Z}$,

$$
\widehat{[f]}(n) \alpha=\int_{\mathbb{T}} \bar{z}^{n}[f](z) \alpha d m=\alpha \int_{\mathbb{T}} \bar{z}^{n} f(z) d m=\alpha \widehat{f}(n)=[\widehat{f}(n)] \alpha,
$$

which gives (c).

The last assertion follows at once from (b) and (c). 
For $\mathcal{X}$ a closed subspace of $D, P_{\mathcal{X}}$ denotes the orthogonal projection from $D$ onto $\mathcal{X}$. Then we have:

Lemma 3.7. If $\operatorname{dim} D<\infty$, then

(a) $L_{s}^{2}(\mathbb{T}, \mathcal{B}(D, E))=L_{\mathcal{B}(D, E)}^{2}$;

(b) $H_{s}^{2}(\mathbb{T}, \mathcal{B}(D, E))=H_{\mathcal{B}(D, E)}^{2}$,

where the equalities are set-theoretic.

Proof. (a) Let $d:=\operatorname{dim} D<\infty$. It follows from (3.1) that $L_{\mathcal{B}(D, E)}^{2} \subseteq$ $L_{s}^{2}(\mathcal{B}(D, E))$. For the reverse inclusion, let $\left\{e_{j}\right\}_{j=1}^{d}$ be an orthonormal basis of $D$. Suppose $\Phi \in L_{s}^{2}(\mathcal{B}(D, E))$. Then

$$
\phi_{j}(z) \equiv \Phi(z) e_{j} \in L_{E}^{2} \quad(j=1,2, \cdots, d) .
$$

It thus follows from Lemma 3.6 that $\left[\phi_{j}\right] \in L_{\mathcal{B}(\mathbb{C}, E)}^{2}$. For $j=1,2, \cdots, d$, define $\Phi_{j}: \mathbb{T} \rightarrow \mathcal{B}(D, E)$ by

$$
\Phi_{j}:=\left[\phi_{j}\right] P_{D_{j}} \quad\left(\mathbb{C} \cong D_{j}:=\bigvee e_{j}\right)
$$

Since $\left[\phi_{j}\right]$ is strongly measurable, it is easy to show that $\Phi_{j}$ is strongly measurable for each $j=1,2, \cdots$. It follows from Lemma 3.6 that

$$
\begin{aligned}
\left\|\Phi_{j}\right\|_{L_{\mathcal{B}(D, E)}^{2}}^{2} & =\int_{\mathbb{T}}\left\|\Phi_{j}(z)\right\|_{\mathcal{B}(D, E)}^{2} d m(z) \\
& =\int_{\mathbb{T}}\left\|\left[\phi_{j}\right](z)\right\|_{\mathcal{B}(\mathbb{C}, E)}^{2} d m(z) \\
& =\left\|\left[\phi_{j}\right]\right\|_{L_{\mathcal{B}(\mathbb{C}, E)}^{2}}^{2} \\
& =\left\|\phi_{j}\right\|_{L_{E}^{2}}^{2} \\
& <\infty .
\end{aligned}
$$

Thus $\Phi_{j} \in L_{\mathcal{B}(D, E)}^{2}$, and hence $\Phi=\sum_{j=1}^{d} \Phi_{j} \in L_{\mathcal{B}(D, E)}^{2}$. This proves (a).

(b) This follows from Lemma 2.2 and (a).

To proceed, we define a "boundary function" $b \Phi$ for each function $\Phi \in H_{s}^{2}(\mathbb{D}, \mathcal{B}(D, E))$ with $\operatorname{dim} D<\infty$. In this case, we may assume that $D=\mathbb{C}^{d}$.

Let $\Phi \in H_{s}^{2}(\mathbb{D}, \mathcal{B}(D, E))$ and $\left\{e_{j}\right\}_{j=1}^{d}$ be the canonical basis for $\mathbb{C}^{d}$. Then $\phi_{j}(\zeta) \equiv \Phi(\zeta) e_{j} \in H^{2}(\mathbb{D}, E)$. Thus we have

$$
\phi_{j}(z) \equiv\left(b \phi_{j}\right)(z):=\lim _{r z \rightarrow z} \phi_{j}(r z) \in H_{E}^{2} .
$$

It follows from Lemma 3.6 that for each $j=1,2,3, \cdots, d$,

$$
\left[\phi_{j}\right] \in H_{\mathcal{B}(\mathbb{C}, E)}^{2}=H_{s}^{2}(\mathbb{T}, \mathcal{B}(\mathbb{C}, E)),
$$

where $\left[\phi_{j}\right](z) \alpha:=\alpha \phi_{j}(z)$ for all $\alpha \in \mathbb{C}$. Note that there exists a subset $\sigma \subset \mathbb{T}$ with $m(\sigma)=0$ such that

$$
\phi_{j}(z) \in E \quad \text { for each } z \in \mathbb{T}_{0} \equiv \mathbb{T} \backslash \sigma .
$$

Define a function $b$ on $H_{s}^{2}(\mathbb{D}, \mathcal{B}(D, E))$ by

$$
(b \Phi)(z):=\left[\left[\phi_{1}\right](z),\left[\phi_{2}\right](z), \cdots,\left[\phi_{d}\right](z)\right] \quad\left(z \in \mathbb{T}_{0}\right) .
$$


Then we have that for all $x \in D$,

$$
(b \Phi)(z) x=\lim _{r z \rightarrow z} \Phi(r z) x \in E \quad\left(z \in \mathbb{T}_{0}\right) .
$$

A straightforward calculation shows that $(b \Phi)(z)$ is a linear mapping from $D$ into $E$ for almost all $z \in \mathbb{T}$.

We thus have:

Theorem 3.8. If $\operatorname{dim} D<\infty$, then the function $b$ defined by (3.9) is a linear bijection from $H_{s}^{2}(\mathbb{D}, \mathcal{B}(D, E))$ onto $H_{s}^{2}(\mathbb{T}, \mathcal{B}(D, E))$.

Proof. Let $d:=\operatorname{dim} D<\infty$. Then we may assume that $D=\mathbb{C}^{d}$. Let $\left\{e_{j}\right\}_{j=1}^{d}$ be the canonical basis for $\mathbb{C}^{d}$ and $\mathbb{T}_{0}$ be defined as the above.

(1) $b$ is well-defined: Let $\Phi \in H_{s}^{2}\left(\mathbb{D}, \mathcal{B}\left(\mathbb{C}^{d}, E\right)\right)$. Then it follows from (3.8) that for each $z_{0} \in \mathbb{T}_{0}$,

$$
\left\|(b \Phi)\left(z_{0}\right)\right\|_{\mathcal{B}\left(\mathbb{C}^{d}, E\right)} \leq \sum_{n=1}^{d}\left\|\phi_{j}\left(z_{0}\right)\right\|_{E}<\infty
$$

which implies that $(b \Phi)\left(z_{0}\right)$ is bounded for each $z_{0} \in \mathbb{T}_{0}$. If $x \equiv\left(x_{1}, x_{2}, \cdots, x_{d}\right)^{t} \in$ $\mathbb{C}^{d}$, then

$$
(b \Phi)(z) x=\sum_{n=1}^{d} x_{j} \phi_{j}(z) \in H_{E}^{2},
$$

which implies that $b \Phi \in H_{s}^{2}\left(\mathcal{B}\left(\mathbb{C}^{d}, E\right)\right)$, and hence $b$ is well-defined.

(2) $b$ is linear: Immediate from a direct calculation.

(3) $b$ is one-one: Let $\Phi, \Psi \in H_{s}^{2}\left(\mathbb{D}, \mathcal{B}\left(\mathbb{C}^{d}, E\right)\right)$. If $b \Phi=b \Psi$, then it follows that for each $x \in \mathbb{C}^{d}$ and $r z \in \mathbb{D}$,

$$
\begin{aligned}
\Phi(r z) x & =\left((b \Phi) x * P_{r}\right)(z) \\
& =\int_{0}^{2 \pi} P_{r}(\theta-t)(b \Phi)\left(e^{i t}\right) x d m(t) \\
& =\int_{0}^{2 \pi} P_{r}(\theta-t)(b \Psi)\left(e^{i t}\right) x d m(t) \\
& =\Psi(r z) x \quad\left(z=e^{i \theta}\right),
\end{aligned}
$$

which gives the result.

(4) $b$ is onto: Let $A \in H_{s}^{2}\left(\mathbb{T}, \mathcal{B}\left(\mathbb{C}^{d}, E\right)\right)$. Then $A(z) e_{j} \in H_{E}^{2}$ for all $j=$ $1,2, \cdots, d$. For each $j=1,2, \cdots, d$, let

$$
\phi_{j}(r z):=\left(A e_{j} * P_{r}\right)(z) \in H^{2}(\mathbb{D}, E)
$$

and define

$$
\Phi(\zeta):=\left[\phi_{1}(\zeta), \phi_{2}(\zeta), \cdots, \phi_{d}(\zeta)\right] \quad(\zeta:=r z)
$$


Then $\Phi \in H_{s}^{2}\left(\mathbb{D}, \mathcal{B}\left(\mathbb{C}^{d}, E\right)\right)$. It follows from (3.10) that for all $x=\left(x_{1}, x_{2}, \cdots, x_{d}\right)^{t} \in$ $\mathbb{C}^{d}$ and for almost all $z \in \mathbb{T}$,

$$
\begin{aligned}
(b \Phi)(z) x & =\lim _{r z \rightarrow z} \Phi(r z) x \\
& =\lim _{r z \rightarrow z} \sum_{j=1}^{d} x_{j} \phi_{j}(r z) \\
& =\sum_{j=1}^{d} x_{j} A(z) e_{j} \\
& =A(z) x
\end{aligned}
$$

which implies that $b$ is onto. This completes the proof.

We thus have:

Corollary 3.9. If $\operatorname{dim} D<\infty$, then the function $b$ defined by (3.9) is an isometric bijection from $H^{2}(\mathbb{D}, \mathcal{B}(D, E))$ onto $H_{\mathcal{B}(D, E)}^{2}$.

Proof. By Theorem 3.8 together with Theorem 3.3 and Lemma 3.7 the function $b$ defined by (3.9) is a linear bijection from $H^{2}(\mathbb{D}, \mathcal{B}(D, E))$ onto $H_{\mathcal{B}(D, E)}^{2}$. In view of the Banach space-valued version of the usual Hardy space theory (cf. [Ni2, Theorem 3.11.6]), it suffices to show that

$$
\Phi\left(r e^{i t}\right)=\left(b \Phi * P_{r}\right)\left(e^{i t}\right)
$$

Indeed, if $z \in \mathbb{T}, r \in(0,1)$, and $x \in D$, then

$$
\begin{aligned}
\left(b \Phi * P_{r}\right)\left(e^{i t}\right) x & =\left(\int_{0}^{2 \pi} P_{r}(\theta-t)(b \Phi)\left(e^{i t}\right) d m(t)\right) x \\
& =\int_{0}^{2 \pi} P_{r}(\theta-t)(b \Phi)\left(e^{i t}\right) x d m(t) \quad \text { (by Lemma 2.2) } \\
& =\Phi\left(r e^{i t}\right) x,
\end{aligned}
$$

which gives 3.11.

According to the convention of the usual Hardy space theory, we will identify $b \Phi$ with $\Phi \in H^{2}(\mathbb{D}, \mathcal{B}(D, E))$. In this sense, we eventually have:

Corollary 3.10. If $\operatorname{dim} D<\infty$, then

$$
H_{s}^{2}(\mathbb{D}, \mathcal{B}(D, E))=H^{2}(\mathbb{D}, \mathcal{B}(D, E))=H_{\mathcal{B}(D, E)}^{2}=H_{s}^{2}(\mathbb{T}, \mathcal{B}(D, E)),
$$

where the first and last equalities are set-theoretic, while the second equality establishes an isometric isomorphism.

Proof. This follows from Theorem 3.3. Lemma 3.7, and Corollary 3.9.

A function $\Delta \in H^{\infty}(\mathcal{B}(D, E))$ is called an inner function with values in $\mathcal{B}(D, E)$ if $\Delta(z)$ is an isometric operator from $D$ into $E$ for almost all $z \in \mathbb{T}$, i.e., $\Delta^{*} \Delta=I_{D}$ a.e. on $\mathbb{T}$. $\Delta$ is called a two-sided inner function if $\Delta \Delta^{*}=I_{E}$ a.e. on $\mathbb{T}$ and $\Delta^{*} \Delta=I_{D}$ a.e. on $\mathbb{T}$. If $\Delta$ is an inner function with values in $\mathcal{B}(D, E)$, we may 
assume that $D$ is a subspace of $E$, and if further $\Delta$ is two-sided inner then we may assume that $D=E$.

We write $\mathcal{P}_{D}$ for the set of all polynomials with values in $D$, i.e., $p(z)=$ $\sum_{k=0}^{n} \widehat{p}(k) z^{k}$, where $\widehat{p}(k) \in D$. If $F$ is a strong $H^{2}$-function with values in $\mathcal{B}(D, E)$, then the function $F p$ belongs to $H_{E}^{2}$ for all $p \in \mathcal{P}_{D}$. The strong $H^{2}$-function $F$ is called outer if $\operatorname{cl} F \mathcal{P}_{D}=H_{E}^{2}$. We then have an analogue of the scalar factorization theorem:

Inner-Outer Factorization for strong $H^{2}$-functions (cf. [Ni1, Corollary I.9]). Every strong $H^{2}$-function $F$ with values in $\mathcal{B}(D, E)$ can be expressed in the form

$$
F=F^{i} F^{e},
$$

where $F^{e}$ is an outer function with values in $\mathcal{B}\left(D, E^{\prime}\right)$ and $F^{i}$ is an inner function with values in $\mathcal{B}\left(E^{\prime}, E\right)$ for some subspace $E^{\prime}$ of $E$.

For a function $\Phi: \mathbb{T} \rightarrow \mathcal{B}(D, E)$, write

$$
\breve{\Phi}(z):=\Phi(\bar{z}), \quad \widetilde{\Phi}:=\breve{\Phi}^{*} .
$$

We call $\breve{\Phi}$ the flip of $\Phi$. For $\Phi \in L_{s}^{2}(\mathcal{B}(D, E))$, we denote by $\breve{\Phi}_{-} \equiv \mathbb{P}_{-} \Phi$ and $\Phi_{+} \equiv \mathbb{P}_{+} \Phi$ the functions

$$
\begin{array}{llll}
\left(\left(\mathbb{P}_{-} \Phi\right)(\cdot)\right) x:=P_{-}(\Phi(\cdot) x) & \text { a.e. on } \mathbb{T} & (x \in D) ; \\
\left(\left(\mathbb{P}_{+} \Phi\right)(\cdot)\right) x:=P_{+}(\Phi(\cdot) x) & \text { a.e. on } \mathbb{T} & (x \in D),
\end{array}
$$

where $P_{+}$and $P_{-}$are the orthogonal projections from $L_{E}^{2}$ onto $H_{E}^{2}$ and $L_{E}^{2} \ominus H_{E}^{2}$, respectively. Then we may write $\Phi \equiv \breve{\Phi}_{-}+\Phi_{+}$. Note that if $\Phi \in L_{s}^{2}(\mathcal{B}(D, E))$, then $\Phi_{+}, \Phi_{-} \in H_{s}^{2}(\mathcal{B}(D, E))$.

In the sequel, we will often encounter the adjoints of inner matrix functions. If $\Delta$ is a two-sided inner matrix function, it is easy to show that $\Delta^{*}$ is of bounded type, i.e., all entries of $\Delta^{*}$ are of bounded type (see p. 3). We may predict that if $\Delta$ is an inner matrix function then $\Delta^{*}$ is of bounded type. However the following example shows that this is not the case.

EXAMPLE 3.11. Let $h(z):=e^{\frac{1}{z-3}}$. Then $h \in H^{\infty}$ and $\bar{h}$ is not of bounded type. Let

$$
f(z):=\frac{h(z)}{\sqrt{2}|| h \|_{\infty}}
$$

Clearly, $\bar{f}$ is not of bounded type. Let $h_{1}(z):=\sqrt{1-|f(z)|^{2}}$. Then $h_{1} \in L^{\infty}$ and $\left|h_{1}\right| \geq \frac{1}{\sqrt{2}}$. Thus there exists an outer function $g$ such that $\left|h_{1}\right|=|g|$ a.e. on $\mathbb{T}$ (see [Do1, Corollary 6.25]). Put

$$
\Delta:=\left[\begin{array}{l}
f \\
g
\end{array}\right] \quad\left(f, g \in H^{\infty}\right)
$$

Then $\Delta^{*} \Delta=|f|^{2}+|g|^{2}=|f|^{2}+\left|h_{1}\right|^{2}=1$ a.e. on $\mathbb{T}$, which implies that $\Delta$ is an inner function. Note that $\Delta^{*}$ is not necessarily of bounded type.

For a function $\Phi \in H_{s}^{2}(\mathcal{B}(D, E))$, we say that an inner function $\Delta$ with values in $\mathcal{B}\left(D^{\prime}, E\right)$ is a left inner divisor of $\Phi$ if $\Phi=\Delta A$ for $A \in H_{s}^{2}\left(\mathcal{B}\left(D, D^{\prime}\right)\right)$. For $\Phi \in H_{s}^{2}\left(\mathcal{B}\left(D_{1}, E\right)\right)$ and $\Psi \in H_{s}^{2}\left(\mathcal{B}\left(D_{2}, E\right)\right)$, we say that $\Phi$ and $\Psi$ are left coprime if the only common left inner divisor of both $\Phi$ and $\Psi$ is a unitary operator. Also, we say that $\Phi$ and $\Psi$ are right coprime if $\widetilde{\Phi}$ and $\widetilde{\Psi}$ are left coprime. Left or right 
coprime-ness seems to be somewhat delicate problem. Left or right coprime-ness for matrix-valued functions was developed in CHKL, CHL1, CHL2, CHL3, and $\mathbf{F F}$.

LEMMA 3.12. If $\Theta$ is a two-sided inner function, then any left inner divisor of $\Theta$ is two-sided inner.

Proof. Suppose that $\Theta$ is a two-sided inner function with values in $\mathcal{B}(E)$ and $\Delta$ is a left inner divisor, with values in $\mathcal{B}\left(E^{\prime}, E\right)$, of $\Theta$. Then we may write $\Theta=\Delta A$ for some $A \in H_{s}^{2}\left(\mathcal{B}\left(E, E^{\prime}\right)\right)$. Since $\Theta$ is two-sided inner, it follows that $I_{E}=\Theta \Theta^{*}=\Delta A A^{*} \Delta^{*}$ a.e. on $\mathbb{T}$, so that $I_{E^{\prime}}=\Delta^{*} \Delta=A A^{*}$ a.e. on $\mathbb{T}$. Thus $I_{E}=\Delta \Delta^{*}$ a.e. on $\mathbb{T}$, and hence $\Delta$ is two-sided inner.

LEMma 3.13. If $\Phi \in L^{\infty}(\mathcal{B}(D, E))$, then $\Phi^{*} \in L^{\infty}(\mathcal{B}(E, D))$. In this case,

$$
\widehat{\Phi^{*}}(-n)=\widehat{\widetilde{\Phi}}(n)=\widehat{\Phi}(n)^{*} \quad(n \in \mathbb{Z}) .
$$

In particular, $\Phi \in H^{\infty}(\mathcal{B}(D, E))$ if and only if $\widetilde{\Phi} \in H^{\infty}(\mathcal{B}(E, D))$.

Proof. Suppose $\Phi \in L^{\infty}(\mathcal{B}(D, E))$. Then

$$
\operatorname{ess} \sup _{z \in \mathbb{T}}|| \Phi^{*}(z)\left\|=\operatorname{ess} \sup _{z \in \mathbb{T}}\right\| \Phi(z) \|<\infty
$$

which together with Lemma 2.1 implies $\Phi^{*} \in L^{\infty}(\mathcal{B}(E, D))$. The first equality of the assertion (3.12) comes from the definition. For the second equality, observe that for each $x \in D, y \in E$ and $n \in \mathbb{Z}$,

$$
\begin{aligned}
\langle\widehat{\Phi}(n) x, y\rangle & =\left\langle\int_{\mathbb{T}} \bar{z}^{n} \Phi(z) x d m(z), y\right\rangle \\
& =\int_{\mathbb{T}}\left\langle\bar{z}^{n} \Phi(z) x, y\right\rangle d m(z) \quad(\text { by }(2.3)) \\
& =\int_{\mathbb{T}}\left\langle x, \bar{z}^{n} \widetilde{\Phi}(z) y\right\rangle d m(z) \\
& =\langle x, \widehat{\widetilde{\Phi}}(n) y\rangle .
\end{aligned}
$$

Lemma 3.14. Let $1 \leq p<\infty$. If $\Phi \in L^{\infty}(\mathcal{B}(D, E))$, then $\Phi L_{s}^{p}\left(\mathcal{B}\left(E^{\prime}, D\right)\right) \subseteq$ $L_{s}^{p}\left(\mathcal{B}\left(E^{\prime}, E\right)\right)$. Also, if $\Phi \in H^{\infty}(\mathcal{B}(D, E))$, then $\Phi H_{s}^{2}\left(\mathcal{B}\left(E^{\prime}, D\right)\right) \subseteq H_{s}^{2}\left(\mathcal{B}\left(E^{\prime}, E\right)\right)$.

Proof. Suppose that $\Phi \in L^{\infty}(\mathcal{B}(D, E))$ and $A \in L_{s}^{p}\left(\mathcal{B}\left(E^{\prime}, D\right)\right)$. Let $x \in E^{\prime}$ be arbitrary. Then we have $A(z) x \in L_{D}^{p}$. Let $\left\{d_{k}\right\}_{k \geq 1}$ be an orthonormal basis for $D$. Thus we may write

$$
A(z) x=\sum_{k \geq 1}\left\langle A(z) x, d_{k}\right\rangle d_{k} \quad \text { for almost all } z \in \mathbb{T} .
$$

Thus it follows that for all $y \in E$,

$$
\langle\Phi(z) A(z) x, y\rangle=\sum_{k \geq 1}\left\langle A(z) x, d_{k}\right\rangle\left\langle\Phi(z) d_{k}, y\right\rangle,
$$


which implies that $\Phi A$ is WOT measurable. On the other hand, since $\Phi \in$ $L^{\infty}(\mathcal{B}(D, E))$, it follows that

$$
\int_{\mathbb{T}}\|(\Phi A)(z) x\|_{E}^{p} d m(z) \leq\|\Phi\|_{\infty}^{p} \int_{\mathbb{T}}\|A(z) x\|_{D}^{p} d m(z)<\infty \quad\left(x \in E^{\prime}\right),
$$

which implies that $\Phi A \in L_{s}^{p}\left(\mathcal{B}\left(E^{\prime}, E\right)\right)$. This proves the first assertion. For the second assertion, suppose $\Phi \in H^{\infty}(\mathcal{B}(D, E))$ and $A \in H_{s}^{2}\left(\mathcal{B}\left(E^{\prime}, D\right)\right)$. Then $\Phi A \in L_{s}^{2}\left(\mathcal{B}\left(E^{\prime}, E\right)\right)$. Assume to the contrary that $\Phi A \notin H_{s}^{2}\left(\mathcal{B}\left(E^{\prime}, E\right)\right)$. Thus, there exists $n_{0}>0$ such that $\widehat{\Phi A}\left(-n_{0}\right) \neq 0$. Thus for some $x_{0} \in E^{\prime}$,

$$
\int_{\mathbb{T}} z^{n_{0}} \Phi(z) A(z) x_{0} d m(z) \neq 0 .
$$

Then by (2.3), there exists a nonzero $y_{0} \in E$ such that

$$
0 \neq\left\langle\int_{\mathbb{T}} z^{n_{0}} \Phi(z) A(z) x_{0} d m(z), y_{0}\right\rangle=\int_{\mathbb{T}}\left\langle A(z) x_{0}, \bar{z}^{n_{0}} \Phi^{*}(z) y_{0}\right\rangle d m(z) .
$$

On the other hand, since $\Phi \in H^{\infty}(\mathcal{B}(D, E))$, it follows from Lemma 3.13 that $\widehat{\Phi^{*}}\left(n_{0}\right)=\widehat{\Phi}\left(-n_{0}\right)^{*}=0$. Thus it follows from (2.3) that

$$
0=\left\langle\widehat{\Phi^{*}}\left(n_{0}\right) y_{0}, A(z) x_{0}\right\rangle=\int_{\mathbb{T}}\left\langle\bar{z}^{n_{0}} \Phi^{*}(z) y_{0}, A(z) x_{0}\right\rangle d m(z),
$$

a contradiction.

Corollary 3.15. Let $1 \leq p<\infty$. If $\Phi \in L^{\infty}(\mathcal{B}(D, E))$, then $\Phi L_{D}^{p} \subseteq L_{E}^{p}$. Also, if $\Phi \in H^{\infty}(\mathcal{B}(D, E))$, then $\Phi H_{D}^{2} \subseteq H_{E}^{2}$.

Proof. Suppose that $\Phi \in L^{\infty}(\mathcal{B}(D, E))$. For $f \in L_{D}^{P}$, we can see that $[\Phi f]=$ $\Phi[f]$. The result thus follows from Lemma 3.6 and Lemma 3.14

For an inner function $\Delta \in H^{\infty}\left(\mathcal{B}\left(E^{\prime}, E\right)\right), \mathcal{H}(\Delta)$ denotes the orthogonal complement of the subspace $\Delta H_{E^{\prime}}^{2}$ in $H_{E}^{2}$, i.e.,

$$
\mathcal{H}(\Delta):=H_{E}^{2} \ominus \Delta H_{E^{\prime}}^{2}
$$

The space $\mathcal{H}(\Delta)$ is often called a model space or a de Branges-Rovnyak space (cf. dR, [Sa, [SFBK]).

We then have:

Corollary 3.16. Let $\Delta$ be an inner function with values in $\mathcal{B}(D, E)$. Then $f \in \mathcal{H}(\Delta)$ if and only if $f \in H_{E}^{2}$ and $\Delta^{*} f \in L_{D}^{2} \ominus H_{D}^{2}$.

Proof. Let $f \in H_{E}^{2}$. By Lemma 3.13 and Corollary 3.15, $\Delta^{*} f \in L_{D}^{2}$. Then $f \in \mathcal{H}(\Delta)$ if and only if $\langle f, \Delta g\rangle=0$ for all $g \in H_{D}^{2}$ if and only if $\left\langle\Delta^{*} f, g\right\rangle=0$ for all $g \in H_{D}^{2}$, which gives the result. 



\section{CHAPTER 4}

\section{The Beurling-Lax-Halmos Theorem}

In this chapter we introduce the Beurling-Lax-Halmos Theorem and the DouglasShapiro-Shields factorization. Then we coin the new notions of complementary factor of an inner function, degree of non-cyclicity, strong $L^{2}$-functions of bounded type, and meromorphic pseudo-continuation of bounded type for operator-valued functions.

\section{$\S$ 4.1. The Beurling-Lax-Halmos Theorem}

We first review a few essential facts for (vectorial) Toeplitz operators and (vectorial) Hankel operators, and for that we will use BS, Do1, Do2, MR, Ni1, Ni2, and $\left[\mathbf{P e}\right.$ for general references. For $\Phi \in L_{s}^{2}(\mathcal{B}(D, E))$, the Hankel operator $H_{\Phi}$ : $H_{D}^{2} \rightarrow H_{E}^{2}$ is a densely defined operator defined by

$$
H_{\Phi} p:=J P_{-}(\Phi p) \quad\left(p \in \mathcal{P}_{D}\right),
$$

where $J$ denotes the unitary operator from $L_{E}^{2}$ to $L_{E}^{2}$ given by $(J g)(z):=\bar{z} g(\bar{z})$ for $g \in L_{E}^{2}$. Also a Toeplitz operator $T_{\Phi}: H_{D}^{2} \rightarrow H_{E}^{2}$ is a densely defined operator defined by

$$
T_{\Phi} p:=P_{+}(\Phi p) \quad\left(p \in \mathcal{P}_{D}\right) .
$$

The following lemma gives a characterization of bounded Hankel operators on $H_{D}^{2}$.

Lemma 4.1. [Pe, Theorem 2.2] Let $\Phi \in L_{s}^{2}(B(D, E))$. Then $H_{\Phi}$ is extended to a bounded operator on $H_{D}^{2}$ if and only if there exists a function $\Psi \in L^{\infty}(\mathcal{B}(D, E))$ such that $\widehat{\Psi}(n)=\widehat{\Phi}(n)$ for $n<0$ and

$$
\left\|H_{\Phi}\right\|=\operatorname{dist}_{L^{\infty}}\left(\Psi, H^{\infty}(\mathcal{B}(D, E)) .\right.
$$

The following basic properties can be easily derived: If $D, E$, and $D^{\prime}$ are separable complex Hilbert spaces and $\Phi \in L^{\infty}(\mathcal{B}(D, E))$, then

$$
\begin{aligned}
& T_{\Phi}^{*}=T_{\Phi^{*}}, H_{\Phi}^{*}=H_{\widetilde{\Phi}} ; \\
& H_{\Phi} T_{\Psi}=H_{\Phi \Psi} \quad \text { if } \Psi \in H^{\infty}\left(\mathcal{B}\left(D^{\prime}, D\right)\right) ; \\
& H_{\Psi \Phi}=T_{\widetilde{\Psi}}^{*} H_{\Phi} \quad \text { if } \Psi \in H^{\infty}\left(\mathcal{B}\left(E, D^{\prime}\right)\right) .
\end{aligned}
$$

A shift operator $S_{E}$ on $H_{E}^{2}$ is defined by

$$
\left(S_{E} f\right)(z):=z f(z) \text { for each } f \in H_{E}^{2} \text {. }
$$

Thus we may write $S_{E}=T_{z I_{E}}$. 
The following theorem is a fundamental result in modern operator theory.

The Beurling-Lax-Halmos Theorem. Beu, Lax, Ha1, FF], Pe A subspace $M$ of $H_{E}^{2}$ is invariant for the shift operator $S_{E}$ on $H_{E}^{2}$ if and only if

$$
M=\Delta H_{E^{\prime}}^{2},
$$

where $E^{\prime}$ is a subspace of $E$ and $\Delta$ is an inner function with values in $\mathcal{B}\left(E^{\prime}, E\right)$. Furthermore, $\Delta$ is unique up to a unitary constant right factor, i.e., if $M=\Theta H_{E^{\prime \prime}}^{2}$, where $\Theta$ is an inner function with values in $\mathcal{B}\left(E^{\prime \prime}, E\right)$, then $\Delta=\Theta V$, where $V$ is a unitary operator from $E^{\prime}$ onto $E^{\prime \prime}$.

As customarily done, we say that two inner functions $A, B \in H^{\infty}(\mathcal{B}(D, E))$ are equal if they are equal up to a unitary constant right factor. If $\Phi \in L^{\infty}(\mathcal{B}(D, E))$, then by (4.2) and (4.3),

$$
H_{\Phi^{*}} S_{E}=S_{E}^{*} H_{\Phi^{*}},
$$

which implies that the kernel of the Hankel operator $H_{\Phi^{*}}$ is an invariant subspace of the shift operator $S_{E}$ on $H_{E}^{2}$. Thus, by the Beurling-Lax-Halmos Theorem,

$$
\operatorname{ker} H_{\Phi^{*}}=\Delta H_{E^{\prime}}^{2}
$$

for some inner function $\Delta$ with values in $\mathcal{B}\left(E^{\prime}, E\right)$. We note that $E^{\prime}$ may be the zero space and $\Delta$ need not be two-sided inner.

We however have:

LEMmA 4.2. If $\Phi \in L^{\infty}(\mathcal{B}(D, E))$ and $\Delta$ is a two-sided inner function with values in $\mathcal{B}(E)$, then the following are equivalent:

(a) $\operatorname{ker} H_{\Phi^{*}}=\Delta H_{E}^{2}$;

(b) $\Phi=\Delta A^{*}$, where $A \in H^{\infty}(\mathcal{B}(E, D))$ is such that $\Delta$ and $A$ are right coprime.

Proof. Let $\Phi \in L^{\infty}(\mathcal{B}(D, E))$ and $\Delta$ be a two-sided inner function with values in $\mathcal{B}(E)$.

$(\mathrm{a}) \Rightarrow(\mathrm{b})$ : Suppose ker $H_{\Phi^{*}}=\Delta H_{E}^{2}$. If we put $A:=\Phi^{*} \Delta \in H^{\infty}(\mathcal{B}(E, D))$, then $\Phi=\Delta A^{*}$. We now claim that $\Delta$ and $A$ are right coprime. To see this, suppose $\Omega$ is a common left inner divisor, with values in $\mathcal{B}\left(E^{\prime}, E\right)$, of $\widetilde{\Delta}$ and $\widetilde{A}$. Then we may write $\widetilde{\Delta}=\Omega \widetilde{\Delta}_{1}$ and $\widetilde{A}=\Omega \widetilde{A}_{1}$, where $\widetilde{\Delta}_{1} \in H^{\infty}\left(\mathcal{B}\left(E, E^{\prime}\right)\right)$ and $\widetilde{A}_{1} \in H^{\infty}\left(\mathcal{B}\left(D, E^{\prime}\right)\right)$. Since $\Delta$ is two-sided inner, it follows from Lemma 3.12 and Lemma 3.13 that $\Omega$ and $\Delta_{1}$ are two-sided inner. Since $\Phi=\Delta_{1} A_{1}^{*}$, we have

$$
\Delta_{1} H_{E^{\prime}}^{2} \subseteq \operatorname{ker} H_{\Phi^{*}}=\Delta H_{E}^{2}=\Delta_{1} \widetilde{\Omega} H_{E}^{2},
$$

which implies $H_{E^{\prime}}^{2}=\widetilde{\Omega} H_{E}^{2}$. Thus by the Beurling-Lax-Halmos Theorem, $\widetilde{\Omega}$ is a unitary constant and so is $\Omega$. Therefore, $\Delta$ and $A$ are right coprime.

(b) $\Rightarrow(\mathrm{a})$ : Suppose (b) holds. Clearly, $\Delta H_{E}^{2} \subseteq$ ker $H_{\Phi^{*}}$. By the BeurlingLax-Halmos Theorem, ker $H_{\Phi^{*}}=\Theta H_{E^{\prime}}^{2}$ for some inner function $\Theta$, so that $\Delta H_{E}^{2} \subseteq$ $\Theta H_{E^{\prime}}^{2}$. Thus $\Theta$ is a left inner divisor of $\Delta$ (cf. $[\mathbf{F F},[\mathbf{P e}$ ) so that, by Lemma 3.12, we may write $\Delta=\Theta \Delta_{0}$ for some two-sided inner function $\Delta_{0}$ with values in $\mathcal{B}\left(E, E^{\prime}\right)$. Put $G:=\Phi^{*} \Theta \in H^{\infty}\left(\mathcal{B}\left(E^{\prime}, D\right)\right)$. Then $G=A \Delta_{0}^{*}$, and hence, $\widetilde{A}=\widetilde{\Delta}_{0} \widetilde{G}$. But since $\Delta$ and $A$ are right coprime, $\widetilde{\Delta}_{0}$ is a unitary operator, and so is $\Delta_{0}$. Therefore ker $H_{\Phi^{*}}=\Delta H_{E}^{2}$, which proves (a). 
We recall that the factorization in Lemma 4.2(b) is called the (canonical) Douglas-Shapiro-Shields factorization of $\Phi \in L^{\infty}(\mathcal{B}(D, E))$ (see DSS, FB, Fu2]). Consequently, Lemma 4.2 may be rephrased as: If $\Phi \in L^{\infty}(\mathcal{B}(D, E))$, then the following are equivalent:

(a) $\Phi$ admits a Douglas-Shapiro-Shields factorization;

(b) $\operatorname{ker} H_{\Phi^{*}}=\Delta H_{E}^{2}$ for some two-sided inner function $\Delta \in H^{\infty}(\mathcal{B}(E))$.

The following lemma will be frequently used in the sequel.

Complementing Lemma. [Ni1, p. 49, p. 53] Let $\Psi \in H^{\infty}\left(\mathcal{B}\left(E^{\prime}, E\right)\right)$ with $E^{\prime} \subseteq$ $E$ and $\operatorname{dim} E^{\prime}<\infty$, and let $\theta$ be a scalar inner function. Then the following statements are equivalent:

(a) There exists a function $G$ in $H^{\infty}\left(\mathcal{B}\left(E, E^{\prime}\right)\right)$ such that $G \Psi=\theta I_{E^{\prime}}$;

(b) There exist functions $\Phi$ and $\Omega$ in $H^{\infty}(\mathcal{B}(E))$ with $\left.\Phi\right|_{E^{\prime}}=\Psi,\left.\Phi\right|_{\left(E \ominus E^{\prime}\right)}$ being an inner function such that $\Omega \Phi=\Phi \Omega=\theta I_{E}$.

In addition, if $\operatorname{dim} E<\infty$, then (a) and (b) are equivalent to the following statement:

(c) $\operatorname{ess}_{\inf } z \in \mathbb{T} \min \{\|\Psi(z) x\|:\|x\|=1\}>0$.

We recall that if $\Phi$ is a strong $H^{2}$-function with values in $\mathcal{B}(D, E)$, with $\operatorname{dim} E<$ $\infty$, the local rank of $\Phi$ is defined by (cf. [Ni1)

$$
\operatorname{Rank} \Phi:=\max _{\zeta \in \mathbb{D}} \operatorname{rank} \Phi(\zeta),
$$

where $\operatorname{rank} \Phi(\zeta):=\operatorname{dim} \Phi(\zeta)(D)$.

As we have remarked in the Introduction, if $\Phi$ is a strong $L^{2}$-function with values in $\mathcal{B}(D, E)$, then $H_{\stackrel{*}{*}}^{*}$ need not be a Hankel operator. Of course, if $\Phi \in$ $L^{\infty}(\mathcal{B}(D, E))$, then by (4.1), $H_{\overparen{\Phi}}^{*}=H_{\widetilde{\Phi}}=H_{\Phi^{*}}$. By contrast, for a strong $L^{2}$ function $\Phi$ with values in $\mathcal{B}(D, E), H_{\widetilde{\Phi}}^{*} \neq H_{\Phi^{*}}$ in general even though $\Phi^{*}$ is also a strong $L^{2}$-function. We note that if $\Phi^{*}$ is a strong $L^{2}$-function with values in $\mathcal{B}(E, D)$, then ker $H_{\Phi^{*}}$ is possibly trivial because $H_{\Phi^{*}}$ is defined in the dense subset of polynomials in $H_{E}^{2}$. Thus it is much better to deal with $H_{\overparen{\Phi}}^{*}$ in place of $H_{\Phi^{*}}$. Even though $H_{\overparen{\Phi}}^{*}$ need not be a Hankel operator, we can show that the kernel of $H_{\vec{\Phi}}^{*}$ is still of the form $\Delta H_{D^{\prime}}^{2}$ for some inner function $\Delta$. To see this, we observe:

Lemma 4.3. Let $\Phi$ be a strong $L^{2}$-function with values in $\mathcal{B}(D, E)$. Then,

$$
\begin{aligned}
\operatorname{ker} H_{\Phi}^{*}=\left\{f \in H_{E}^{2}: \int_{\mathbb{T}}\left\langle\Phi(z) x, z^{n} f(z)\right\rangle_{E} d m(z)=0\right. & \text { for all } x \in D \\
& \text { and } n=1,2,3, \cdots\} .
\end{aligned}
$$

Proof. Observe that

$$
\begin{aligned}
f \in \operatorname{ker} H_{\breve{\Phi}}^{*} & \Longleftrightarrow\left\langle H_{\breve{\Phi}} p, f\right\rangle_{L_{E}^{2}}=0 \quad \text { for all } p \in \mathcal{P}_{D} \\
& \Longleftrightarrow\langle\breve{\Phi}(z) p(z),(J f)(z)\rangle_{L_{E}^{2}}=0 \quad \text { for all } p \in \mathcal{P}_{D} \\
& \Longleftrightarrow \int_{\mathbb{T}}\left\langle\Phi(\bar{z}) x z^{k}, \bar{z} f(\bar{z})\right\rangle_{E} d m(z)=0 \quad \text { for all } x \in D \text { and } k=0,1,2, \cdots \\
& \Longleftrightarrow \int_{\mathbb{T}}\left\langle\Phi(z) x, z^{n} f(z)\right\rangle_{E} d m(z)=0 \quad \text { for all } x \in D \text { and } n=1,2,3, \cdots
\end{aligned}
$$


which gives the result.

We then have:

Lemma 4.4. If $\Phi$ is a strong $L^{2}$-function with values in $\mathcal{B}(D, E)$, then

$$
\operatorname{ker} H_{\overparen{\Phi}}^{*}=\Delta H_{E^{\prime}}^{2},
$$

where $E^{\prime}$ is a subspace of $E$ and $\Delta$ is an inner function with values in $\mathcal{B}\left(E^{\prime}, E\right)$.

Proof. By Lemma 4.3, if $f \in \operatorname{ker} H_{\overparen{\Phi}}^{*}$, then $z f \in \operatorname{ker} H_{\overparen{\Phi}}^{*}$. Since $\operatorname{ker} H_{\overparen{\Phi}}^{*}$ is always closed, it follows that ker $H_{\overparen{\Phi}}^{*}$ is an invariant subspace for $S_{E}$. Thus, by the Beurling-Lax-Halmos Theorem, there exists an inner function $\Delta$ with values in $\mathcal{B}\left(E^{\prime}, E\right)$ such that $\operatorname{ker} H_{\overparen{\Phi}}^{*}=\Delta H_{E^{\prime}}^{2}$ for a subspace $E^{\prime}$ of $E$.

\section{$\S$ 4.2. Complementary factors of inner functions}

Let $\left\{\Theta_{i} \in H^{\infty}\left(\mathcal{B}\left(E_{i}, E\right)\right): i \in J\right\}$ be a family of inner functions. Then the greatest common left inner divisor $\Theta_{d}$ and the least common left inner multiple $\Theta_{m}$ of the family $\left\{\Theta_{i}: i \in J\right\}$ are the inner functions defined by

$$
\Theta_{d} H_{D}^{2}:=\bigvee_{i \in J} \Theta_{i} H_{E_{i}}^{2} \quad \text { and } \quad \Theta_{m} H_{D^{\prime}}^{2}:=\bigcap_{i \in J} \Theta_{i} H_{E_{i}}^{2} .
$$

By the Beurling-Lax-Halmos Theorem, $\Theta_{d}$ and $\Theta_{m}$ exist, and are unique up to a unitary constant right factor. We write

$$
\Theta_{d} \equiv \text { left-g.c.d. }\left\{\Theta_{i}: i \in J\right\} \quad \text { and } \quad \Theta_{m} \equiv \text { left-l.c.m. }\left\{\Theta_{i}: i \in J\right\} .
$$

If $\Theta_{i}$ is a scalar inner function, we write

$$
\text { g.c.d. }\left\{\Theta_{i}: i \in J\right\} \equiv \text { left-g.c.d. }\left\{\Theta_{i}: i \in J\right\}
$$

and

$$
\text { l.c.m. }\left\{\Theta_{i}: i \in J\right\} \equiv \text { left-l.c.m. }\left\{\Theta_{i}: i \in J\right\} \text {. }
$$

For $\Phi \in L^{\infty}(\mathcal{B}(D, E))$, we symbolically define the kernel of $\Phi$ by

$$
\operatorname{ker} \Phi:=\left\{f \in H_{D}^{2}: \Phi(z) f(z)=0 \text { for almost all } z \in \mathbb{T}\right\} .
$$

Note that the kernel of $\Phi$ consists of functions in $H_{D}^{2}$, but not in $L_{D}^{2}$, such that $\Phi f=0$ a.e. on $\mathbb{T}$. Since $\operatorname{ker} \Phi$ is an invariant subspace for $S_{D}$, it follows from the Beurling-Lax-Halmos Theorem that $\operatorname{ker} \Phi=\Omega H_{D^{\prime}}^{2}$, for some inner function $\Omega \in H^{\infty}\left(D^{\prime}, D\right)$.

Let $\Delta$ be an inner function with values in $\mathcal{B}(D, E)$. If $g \in \operatorname{ker} \Delta^{*}$, then $g \in H_{E}^{2}$, so that by Lemma 3.5 and Lemma 3.6. $[\mathrm{g}]$ is a strong $H^{2}$-function with values in $\mathcal{B}(\mathbb{C}, E)$ (see $\mathrm{p} 19$ for the definition of $[g]$ ). Write

$$
[g]=[g]^{i}[g]^{e} \quad \text { (inner-outer factorization), }
$$

where $[g]^{e}$ is an outer function with values in $\mathcal{B}\left(\mathbb{C}, E^{\prime}\right)$ and $[g]^{i}$ is an inner function with values in $\mathcal{B}\left(E^{\prime}, E\right)$ for some subspace $E^{\prime}$ of $E$. If $g \neq 0$, then $[g]^{e}$ is a nonzero outer function, so that $E^{\prime}=\mathbb{C}$. Thus, $[g]^{i} \in H^{\infty}(\mathcal{B}(\mathbb{C}, E))$. If instead $g=0$, then $E^{\prime}=\{0\}$. Therefore, in this case, $[g]^{i} \in H^{\infty}(\mathcal{B}(\{0\}, E))$.

We then have: 
Lemma 4.5. Let $\Delta$ be an inner function with values in $\mathcal{B}(D, E)$. Then we may write

$$
\operatorname{ker} \Delta^{*}=\Omega H_{D^{\prime}}^{2}
$$

for some inner function $\Omega$ with values in $B\left(D^{\prime}, E\right)$. Put

$$
\Delta_{c}:=\text { left-g.c.d. }\left\{[g]^{i}: g \in \operatorname{ker} \Delta^{*}\right\} .
$$

Then we have

(a) $\Omega=\Delta_{c}$;

(b) $\left[\Delta, \Delta_{c}\right]$ is an inner function with values in $\mathcal{B}\left(D \oplus D^{\prime}, E\right)$;

(c) $\operatorname{ker} H_{\Delta^{*}}=\left[\Delta, \Delta_{c}\right] H_{D \oplus D^{\prime}}^{2} \equiv \Delta H_{D}^{2} \oplus \Delta_{c} H_{D^{\prime}}^{2}$,

where $\left[\Delta, \Delta_{c}\right]$ is obtained by complementing $\Delta_{c}$ to $\Delta$, in other words, $\left[\Delta, \Delta_{c}\right]$ is regarded as a $1 \times 2$ operator matrix.

Definition 4.6. The inner function $\Delta_{c}$ in (4.6) is said to be the complementary factor of the inner function $\Delta$.

Proof of Lemma 4.5. If ker $\Delta^{*}=\{0\}$, then (a) and (b) are trivial. Suppose that $\operatorname{ker} \Delta^{*} \neq\{0\}$. Recall that

$$
\Delta_{c}=\text { left-g.c.d. }\left\{[g]^{i}: g \in \operatorname{ker} \Delta^{*}\right\} \in H^{\infty}\left(\mathcal{B}\left(D^{\prime \prime}, E\right)\right),
$$

where $D^{\prime \prime}$ is a nonzero subspace of $E$. If $g \in \operatorname{ker} \Delta^{*}$, then it follows from (4.5) that

$$
\begin{aligned}
\Delta_{c} H_{D^{\prime \prime}}^{2} & =\bigvee\left\{[g]^{i} H^{2}: g \in \operatorname{ker} \Delta^{*}\right\} \\
& =\bigvee\left\{[g] \mathcal{P}_{\mathbb{C}}: g \in \operatorname{ker} \Delta^{*}\right\} \\
& \subseteq \operatorname{ker} \Delta^{*}=\Omega H_{D^{\prime}}^{2} .
\end{aligned}
$$

For the reverse inclusion, let $0 \neq g \in \operatorname{ker} \Delta^{*}$. Then it follows that

$$
g(z)=[g](z) 1=\left([g]^{i}[g]^{e}\right)(z) 1=[g]^{i}(z)\left([g]^{e}(z) 1\right) \in[g]^{i} H^{2} .
$$

Thus we have

$$
\Omega H_{D^{\prime}}^{2}=\operatorname{ker} \Delta^{*} \subseteq \bigvee\left\{[g]^{i} H^{2}: g \in \operatorname{ker} \Delta^{*}\right\}=\Delta_{c} H_{D^{\prime \prime}}^{2} .
$$

Therefore, by the Beurling-Lax-Halmos Theorem, $\Omega=\Delta_{c}$ and $D^{\prime}=D^{\prime \prime}$, which gives (a). Note that $\Delta^{*} \Delta_{c}=0$. We thus have

$$
\left[\begin{array}{c}
\Delta^{*} \\
\Delta_{c}^{*}
\end{array}\right]\left[\Delta, \Delta_{c}\right]=\left[\begin{array}{cc}
I_{D} & 0 \\
0 & I_{D^{\prime}}
\end{array}\right]
$$

which implies that $\left[\Delta, \Delta_{c}\right]$ is an inner function with values in $\mathcal{B}\left(D \oplus D^{\prime}, E\right)$, which gives (b). For (c), we first note that $\Delta H_{D}^{2}$ and $\operatorname{ker} \Delta^{*}$ are orthogonal and

$$
\Delta H_{D}^{2} \bigoplus \operatorname{ker} \Delta^{*} \subseteq \operatorname{ker} H_{\Delta^{*}} .
$$

For the reverse inclusion, suppose that $f \in H_{E}^{2}$ and $f \notin \Delta H_{D}^{2} \bigoplus \operatorname{ker} \Delta^{*} \equiv M$. Write

$$
f_{1}:=P_{M} f \text { and } f_{2}:=f-f_{1} \neq 0 .
$$


Since $f_{2} \in H_{E}^{2} \ominus M=\mathcal{H}(\Delta) \cap\left(H_{E}^{2} \ominus\right.$ ker $\left.\Delta^{*}\right)$, it follows from Corollary 3.16 that $\Delta^{*} f_{2} \in L_{D}^{2} \ominus H_{D}^{2}$ and $\Delta^{*} f_{2} \neq 0$. We thus have $H_{\Delta^{*}} f=J\left(\Delta^{*} f_{2}\right)$, and hence, $\left\|H_{\Delta^{*}} f\right\|=\left\|\Delta^{*} f_{2}\right\| \neq 0$, which implies that $f \notin$ ker $H_{\Delta^{*}}$. We thus have that

$$
\operatorname{ker} H_{\Delta^{*}}=\Delta H_{D}^{2} \bigoplus \operatorname{ker} \Delta^{*}
$$

Thus it follows from (a) that

$$
\operatorname{ker} H_{\Delta^{*}}=\Delta H_{D}^{2} \bigoplus \Delta_{c} H_{D^{\prime}}^{2}=\left[\Delta, \Delta_{c}\right] H_{D \oplus D^{\prime}}^{2},
$$

which gives (c). This completes the proof.

\section{$\S$ 4.3. The degree of non-cyclicity}

For a subset $F$ of $H_{E}^{2}$, let $E_{F}^{*}$ denote the smallest $S_{E}^{*}$-invariant subspace containing $F$, i.e.,

$$
E_{F}^{*}=\bigvee\left\{S_{E}^{* n} F: n \geq 0\right\} .
$$

Then by the Beurling-Lax-Halmos Theorem, $E_{F}^{*}=\mathcal{H}(\Delta)$ for an inner function $\Delta$ with values in $\mathcal{B}(D, E)$. In general, if $\operatorname{dim} E=1$, then every $S_{E}^{*}$-invariant subspace $M$ admits a cyclic vector, i.e., $M=E_{f}^{*}$ for some $f \in H^{2}$. However, if $\operatorname{dim} E \geq 2$, then this is not such a case. For example, if $M=\mathcal{H}(\Delta)$ with $\Delta=\left[\begin{array}{cc}z & 0 \\ 0 & z\end{array}\right]$, then $M$ does not admit a cyclic vector, i.e., $M \neq E_{f}^{*}$ for any vector $f \in H_{\mathbb{C}^{2}}^{2}$.

If $\Phi \in H_{s}^{2}(\mathcal{B}(D, E))$ and $\left\{d_{k}\right\}_{k \geq 1}$ is an orthonormal basis for $D$, write

$$
\phi_{k}:=\Phi d_{k} \in H_{E}^{2} \cong H_{s}^{2}(\mathcal{B}(\mathbb{C}, E)) .
$$

We then define

$$
\{\Phi\}:=\left\{\phi_{k}\right\}_{k \geq 1} \subseteq H_{E}^{2} .
$$

Hence, $\{\Phi\}$ may be regarded as the set of "column" vectors $\phi_{k}$ (in $H_{E}^{2}$ ) of $\Phi$, in which case we may think of $\Phi$ as an infinite matrix-valued function.

Lemma 4.7. For $\Phi \in H_{s}^{2}(\mathcal{B}(D, E))$, we have

$$
E_{\{\Phi\}}^{*}=\mathrm{cl} \operatorname{ran} H_{\bar{z} \breve{\Phi}}
$$

Remark 4.8. By definition, $\{\Phi\}$ depends on the orthonormal basis of $D$. However, Lemma 4.7 shows that $E_{\{\Phi\}}^{*}$ is independent of a particular choice of the orthonormal basis of $D$ because the right-hand side of (4.8) is independent of the orthonormal basis of $D$.

Proof of Lemma 4.7. We first claim that if $f \in H_{E}^{2}$, then

$$
E_{f}^{*}=\mathrm{cl} \operatorname{ran} H_{[\bar{z} \breve{f}]} .
$$


To see this, observe that for each $k=1,2, \cdots$,

$$
\begin{aligned}
S_{E}^{* k} f & =\bar{z} \sum_{j=0}^{\infty} \widehat{f}(k+j) z^{j+1} \\
& =J\left(\sum_{j=0}^{\infty} \widehat{f}(k+j) \bar{z}^{j+1}\right) \\
& =J P_{-}\left(z^{k-1} \sum_{j=0}^{\infty} \widehat{f}(j) \bar{z}^{j}\right) \\
& =J P_{-}\left(z^{k-1} \breve{f}\right) \\
& =H_{[\bar{z} \breve{f}]} z^{k},
\end{aligned}
$$

which proves (4.9). Let $\left\{d_{k}\right\}_{k \geq 1}$ be an orthonormal basis for $D$, and let $\phi_{k}:=\Phi d_{k}$. Since by (4.9), $E_{\phi_{k}}^{*}=\operatorname{cl} \operatorname{ran} H_{\left[\bar{z} \breve{\phi}_{k}\right]}$ for each $k=1,2,3, \cdots$, it follows that

$$
E_{\{\Phi\}}^{*}=\bigvee \operatorname{ran} H_{\left[\bar{z} \breve{\phi}_{k}\right]}=\mathrm{cl} \operatorname{ran} H_{\bar{z} \widetilde{\Phi}},
$$

which gives the result.

We now introduce:

Definition 4.9. Let $F \subseteq H_{E}^{2}$. The degree of non-cyclicity, denoted by $\operatorname{nc}(F)$, of $F$ is defined by the number

$$
\operatorname{nc}(F):=\sup _{\zeta \in \mathbb{D}} \operatorname{dim}\left\{g(\zeta): g \in H_{E}^{2} \ominus E_{F}^{*}\right\} .
$$

We will often refer to nc $(F)$ as the nc-number of $F$.

Since $E_{F}^{*}$ is an invariant subspace for $S_{E}^{*}$, it follows from the Beurling-LaxHalmos Theorem that $E_{F}^{*}=\mathcal{H}(\Delta)$ for some inner function $\Delta$ with values in $\mathcal{B}(D, E)$. Thus

$$
\mathrm{nc}(F)=\sup _{\zeta \in \mathbb{D}} \operatorname{dim}\left\{g(\zeta): g \in \Delta H_{D}^{2}\right\}=\operatorname{dim} D .
$$

In particular, $\mathrm{nc}(F) \leq \operatorname{dim} E$. We note that $\mathrm{nc}(F)$ may take $\infty$. So it is customary to make the following conventions: (i) if $n$ is real then $n+\infty=\infty$; (ii) $\infty+\infty=\infty$. If $\operatorname{dim} E=r<\infty$, then $\operatorname{nc}(F) \leq r$ for every subset $F \subseteq H_{E}^{2}$. If $F \subseteq H_{E}^{2}$ and $\operatorname{dim} E=r<\infty$, then the degree of cyclicity, denoted by $\operatorname{dc}(F)$, of $F \subseteq H_{E}^{2}$ is defined by the number (cf. $\mathbf{V N}$ )

$$
\operatorname{dc}(F):=r-\operatorname{nc}(F) .
$$

In particular, if $E_{F}^{*}=\mathcal{H}(\Delta)$, then $\Delta$ is two-sided inner if and only if $\operatorname{nc}(F)=r$.

The following theorem gives an answer to Question 1.3 . 
TheOrem 4.10. Let $\Phi$ be a strong $L^{2}$-function with values in $\mathcal{B}(D, E)$. In view of the Beurling-Lax-Halmos Theorem and Lemma 4.4 we may write

$$
E_{\left\{\Phi_{+}\right\}}^{*}=\mathcal{H}(\Delta) \text { and } \operatorname{ker} H_{\overleftrightarrow{\Phi}}^{*}=\Theta H_{E^{\prime}}^{2},
$$

for some inner functions $\Delta$ and $\Theta$ with values in $\mathcal{B}\left(E^{\prime \prime}, E\right)$ and $\mathcal{B}\left(E^{\prime}, E\right)$, respectively. Then

$$
\Delta=\Theta \Delta_{1}
$$

for some two-sided inner function $\Delta_{1}$ with values in $\mathcal{B}\left(E^{\prime \prime}, E^{\prime}\right)$. Hence, in particular,

$$
\operatorname{ker} H_{\breve{\Phi}}^{*}=\Theta H_{E^{\prime}}^{2} \Longleftrightarrow \operatorname{nc}\left\{\Phi_{+}\right\}=\operatorname{dim} E^{\prime} .
$$

Proof. Suppose that ker $H_{\overleftrightarrow{\Phi}}^{*}=\Theta H_{E^{\prime}}^{2}$ for some inner function $\Theta$ with values in $\mathcal{B}\left(E^{\prime}, E\right)$ and $E_{\left\{\Phi_{+}\right\}}^{*}=\mathcal{H}(\Delta)$ for some inner function $\Delta$ with values in $\mathcal{B}\left(E^{\prime \prime}, E\right)$. Then it follows from Lemma 4.7 that

$$
\mathcal{H}(\Delta)=E_{\left\{\Phi_{+}\right\}}^{*}=\operatorname{cl} \operatorname{ran} H_{\bar{z} \breve{\Phi}}=\left(\operatorname{ker} H_{\bar{z} \breve{\Phi}}^{*}\right)^{\perp} .
$$

It thus follows from Lemma 4.3 that

$$
\begin{array}{rlr}
\Delta H_{E^{\prime \prime}}^{2} & =\operatorname{ker} H_{z \breve{\Phi}}^{*} \\
& =\left\{f \in H_{E}^{2}: \int_{\mathbb{T}}\left\langle\Phi(z) x, z^{n} f(z)\right\rangle_{E} d m(z)=0\right. & \text { for all } x \in D \\
& \subseteq\left\{f \in H_{E}^{2}: \int_{\mathbb{T}}\left\langle\Phi(z) x, z^{n} f(z)\right\rangle_{E} d m(z)=0\right. & \text { and } n=0,1,2,3, \cdots\} \\
& \text { for all } x \in D \\
& =\operatorname{ker} H_{\stackrel{\Phi}{*}}^{*}=\Theta H_{E^{\prime}}^{2}, & \text { and } n=1,2,3, \cdots\}
\end{array}
$$

which implies that $\Theta$ is a left inner divisor of $\Delta$. Thus we can write

$$
\Delta=\Theta \Delta_{1}
$$

for some inner function $\Delta_{1} \in H^{\infty}\left(\mathcal{B}\left(E^{\prime \prime}, E^{\prime}\right)\right)$. By the same argument as above, we also have $z \Theta H_{E^{\prime}}^{2} \subseteq \Delta H_{E^{\prime \prime}}^{2}$, so that we may write $z \Theta=\Delta \Delta_{2}$ for some inner function $\Delta_{2} \in H^{\infty}\left(\mathcal{B}\left(E^{\prime}, E^{\prime \prime}\right)\right)$. Therefore by (4.12), we have $z I_{E^{\prime}}=\Delta_{1} \Delta_{2}$, and hence by Lemma 3.12, $\Delta_{1}$ is two-sided inner. This proves (4.10) and in turn (4.11). This completes the proof.

From Theorem 4.10, we get several corollaries.

Corollary 4.11. Let $\Phi$ be a strong $L^{2}$-function with value in $\mathcal{B}(D, E)$. Then the following statements are equivalent:

(a) $E_{\left\{\Phi_{+}\right\}}^{*}=H_{E}^{2}$;

(b) $\operatorname{nc}\left\{\Phi_{+}\right\}=0$;

(c) $\operatorname{ker} H_{\stackrel{\Phi}{*}}^{*}=\{0\}$.

Proof. Immediate from Theorem 4.10 . 
Corollary 4.12. Let $\Delta$ be an inner function with values in $\mathcal{B}(D, E)$. If $\Delta_{c}$ is the complementary factor of $\Delta$, with values in $\mathcal{B}\left(D^{\prime}, E\right)$, then

$$
\operatorname{nc}\{\Delta\}=\operatorname{dim} D+\operatorname{dim} D^{\prime} \text {. }
$$

Proof. Immediate from Lemma 4.5(c) and Theorem 4.10,

COROLlary 4.13. If $\Phi$ is an $n \times m$ matrix $L^{2}$-function, i.e., $\Phi \in L_{M_{n \times m}}^{2}$, then the following are equivalent:

(a) $\Phi$ is of bounded type;

(b) $\operatorname{ker} H_{\Phi}^{*}=\Delta H_{\mathbb{C}^{n}}^{2}$ for some two-sided inner matrix function $\Delta$;

(c) nc $\left\{\Phi_{-}\right\}=n$.

Proof. The equivalence (a) $\Leftrightarrow$ (c) follows from [Ni1, Corollary 2, p. 47] and (2.1), and the equivalence (b) $\Leftrightarrow$ (c) follows at once from Theorem 4.10 .

The equivalence (a) $\Leftrightarrow$ (b) of Corollary 4.13 was known from GHR for the

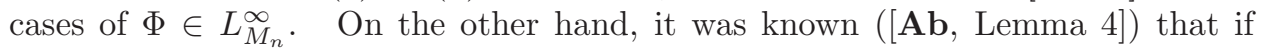
$\phi \in L^{\infty}$, then

$$
\phi \text { is of bounded type } \Longleftrightarrow \operatorname{ker} H_{\phi} \neq\{0\} .
$$

The following corollary shows that (4.13) still holds for $L^{2}$-functions.

Corollary 4.14. If $\phi \in L^{2}$, then $\phi$ is of bounded type if and only if ker $H_{\phi}^{*} \neq$ $\{0\}$.

Proof. Immediate from Corollary 4.13.

Corollary 4.15. If $\Delta$ is an $n \times r$ inner matrix function then the following are equivalent:

(a) $\Delta^{*}$ is of bounded type;

(b) $\breve{\Delta}$ is of bounded type;

(c) $\left[\Delta, \Delta_{c}\right]$ is two-sided inner,

where $\Delta_{c}$ is the complementary factor of $\Delta$.

Proof. The equivalence (a) $\Leftrightarrow(\mathrm{b})$ is trivial. The equivalence (b) $\Leftrightarrow$ (c) follows from Lemma 4.5 and Corollary 4.13 .

The following corollary gives an answer to Question 1.4 .

Corollary 4.16. If $\Delta$ is an $n \times r$ inner matrix function, then $[\Delta, \Omega]$ is inner for some $n \times q(q \geq 1)$ inner matrix function $\Omega$ if and only if

$$
q \leq \operatorname{nc}\{\Delta\}-r .
$$

In particular, $\Delta$ is complemented to a two-sided inner function if and only if $\operatorname{nc}\{\Delta\}=n$. 
Proof. Suppose that $[\Delta, \Omega]$ is an inner matrix function for some $n \times q(q \geq 1)$ inner matrix function $\Omega$. Then

$$
I_{r+q}=[\Delta, \Omega]^{*}[\Delta, \Omega]=\left[\begin{array}{cc}
I_{r} & \Delta^{*} \Omega \\
\Omega^{*} \Delta & I_{q}
\end{array}\right]
$$

which implies that $\Omega H_{\mathbb{C}^{q}}^{2} \subseteq \operatorname{ker} \Delta^{*}$. Since by Lemma 4.5, ker $\Delta^{*}=\Delta_{c} H_{\mathbb{C}^{p}}^{2}$, it follows that $\Omega H_{\mathbb{C}^{q}}^{2} \subseteq \Delta_{c} H_{\mathbb{C}^{p}}^{2}$, so that $\Delta_{c}$ is a left inner divisor of $\Omega$. Thus we can write

$$
\Omega=\Delta_{c} \Omega_{1} \text { for some } p \times q \text { inner matrix function } \Omega_{1} .
$$

Thus we have $q \leq p$. But since by Corollary 4.12, nc $\{\Delta\}=r+p$, it follows that $q \leq \operatorname{nc}\{\Delta\}-r$. For the converse, suppose that $q \leq \operatorname{nc}\{\Delta\}-r$. Then it follows from Corollary 4.12 that the complementary factor $\Delta_{c}$ of $\Delta$ is in $H_{M_{n \times p}}^{\infty}$ for some $p \geq q$. Thus if we take $\Omega:=\left.\Delta_{c}\right|_{\mathbb{C}^{q}}$, then $[\Delta, \Omega]$ is inner.

We give an illuminating example of how to find the nc number.

ExAMPLE 4.17. Let $f$ and $g$ be given in Example 3.11, and let

$$
\Phi:=\left[\begin{array}{lll}
f & f & 0 \\
g & g & 0 \\
0 & 0 & a
\end{array}\right] \quad\left(a \in H^{\infty}\right)
$$

To find the degree of non-cyclicity of $\Phi$, write $\Psi:=\left[\begin{array}{ll}f & f \\ g & g\end{array}\right]$. Then it follows that

$$
\left[\begin{array}{l}
h_{1} \\
h_{2} \\
h_{3}
\end{array}\right] \in \operatorname{ker} H_{\bar{\Phi}}^{*} \Longleftrightarrow\left[\begin{array}{l}
h_{1} \\
h_{2}
\end{array}\right] \in \operatorname{ker} H_{\Psi^{*}} \text { and } h_{3} \in \operatorname{ker} H_{\bar{a}} .
$$

Case 1: If $\bar{a}$ is not of bounded type, then $\operatorname{ker} H_{\overleftrightarrow{\Phi}}^{*}=\left[\begin{array}{ll}f g & 0\end{array}\right]^{t} H^{2}$. By Theorem 4.10, $\operatorname{nc}\{\Phi\}=1$.

Case 2: If $\bar{a}$ is of bounded type of the form $a=\theta \bar{b}$ (coprime), then

$$
\operatorname{ker} H_{\Phi^{*}}^{*}=\left[\begin{array}{ll}
f & 0 \\
g & 0 \\
0 & \theta
\end{array}\right] H_{\mathbb{C}^{2}}^{2}
$$

By Theorem 4.10, nc $\{\Phi\}=2$.

\section{$\S$ 4.4. Strong $L^{2}$-functions of bounded type}

We introduce the notion of "bounded type" for strong $L^{2}$-functions. Recall that a matrix-valued function of bounded type was defined by a matrix whose entries are of bounded type (see p. 3). But this definition is not appropriate for operatorvalued functions, in particular strong $L^{2}$-functions, even though the terminology of "entry" can be properly interpreted. Thus we need a new idea about how to define a "bounded type" strong $L^{2}$-functions, which is equivalent to the condition that each entry is of bounded type when the function is matrix-valued. Our motivation stems from the equivalence $(\mathrm{a}) \Leftrightarrow(\mathrm{b})$ in Corollary 4.13 . 
Definition 4.18. A strong $L^{2}$-function $\Phi$ with values in $\mathcal{B}(D, E)$ is said to be of bounded type if ker $H_{\Phi}^{*}=\Theta H_{E}^{2}$ for some two-sided inner function $\Theta$ with values in $\mathcal{B}(E)$.

On the other hand, in $\mathbf{F B}$, it was shown that if $\Phi$ belongs to $L^{\infty}(\mathcal{B}(D, E))$, then $\Phi$ admits a Douglas-Shapiro-Shields factorization (see p. 29) if and only if $E_{\left\{\Phi_{+}\right\}}^{*}=\mathcal{H}(\Theta)$ for a two-sided inner function $\Theta$. Thus, by Theorem 4.10, we can see that if $\Phi \in L^{\infty}(\mathcal{B}(D, E))$, then

$\breve{\Phi}$ is of bounded type $\Longleftrightarrow \Phi$ admits a Douglas-Shapiro-Shields factorization.

We can prove more:

Lemma 4.19. Let $\Phi$ be a strong $L^{2}$-function with values in $\mathcal{B}(D, E)$. Then the following are equivalent:

(a) $\breve{\Phi}$ is of bounded type;

(b) $E_{\left\{\Phi_{+}\right\}}^{*}=\mathcal{H}(\Delta)$ for some two-sided inner function $\Delta$ with values in $\mathcal{B}(E)$;

(c) $E_{\left\{\Phi_{+}\right\}}^{*} \subseteq \mathcal{H}(\Theta)$ for some two-sided inner function $\Theta$ with values in $\mathcal{B}(E)$;

(d) $\left\{\Phi_{+}\right\} \subseteq \mathcal{H}(\Theta)$ for some two-sided inner function $\Theta$ with values in $\mathcal{B}(E)$;

(e) For $\left\{\varphi_{k_{1}}, \varphi_{k_{2}}, \cdots\right\} \subseteq\{\Phi\}$, write $\Psi \equiv\left[\varphi_{k_{1}}, \varphi_{k_{2}}, \cdots\right]$. Then $\breve{\Psi}$ is of bounded type.

Proof. (a) $\Rightarrow(\mathrm{b})$ : Suppose that $\breve{\Phi}$ is of bounded type. Then $\operatorname{ker} H_{\breve{\Phi}}^{*}=\Theta H_{E}^{2}$ for some two-sided inner function $\Theta$ with values in $\mathcal{B}(E)$. It thus follows from Theorem 4.10 that $E_{\left\{\Phi_{+}\right\}}^{*}=\mathcal{H}(\Delta)$ for some two-sided inner function $\Delta$ with values in $\mathcal{B}(E)$.

(b) $\Rightarrow(\mathrm{c}),(\mathrm{c}) \Rightarrow(\mathrm{d})$ : Clear.

(d) $\Rightarrow$ (e): Suppose that $\left\{\varphi_{k_{1}}, \varphi_{k_{2}}, \cdots\right\} \subseteq\{\Phi\}$ and $\left\{\Phi_{+}\right\} \subseteq \mathcal{H}(\Theta)$ for some twosided inner function $\Theta \in H^{\infty}(\mathcal{B}(E))$. Write $\Psi \equiv\left[\varphi_{k_{1}}, \varphi_{k_{2}}, \cdots\right]$. Then $\left\{\Psi_{+}\right\} \subseteq$ $\mathcal{H}(\Theta)$, so that $E_{\left\{\Psi_{+}\right\}}^{*} \subseteq \mathcal{H}(\Theta)$. Suppose that $E_{\left\{\Psi_{+}\right\}}^{*}=\mathcal{H}(\Delta)$ for some inner function $\Delta$ with values in $\mathcal{B}\left(D^{\prime}, E\right)$. Thus $\Theta H_{E}^{2} \subseteq \Delta H_{D^{\prime}}^{2}$, so that by Lemma 3.12 $\Delta$ is two-sided inner. Thus, by Theorem 4.10, ker $H_{\breve{\Psi}}^{*}=\Omega H_{E}^{2}$ for some two-sided inner function $\Omega$ with values in $\mathcal{B}(E)$, so that $\Psi$ is of bounded type.

(e) $\Rightarrow($ a): Clear.

Corollary 4.20. Let $\Delta$ be an inner function with values in $\mathcal{B}(D, E)$. Then

$$
\breve{\Delta} \text { is of bounded type } \Longleftrightarrow\left[\Delta, \Delta_{c}\right] \text { is two-sided inner, }
$$

where $\Delta_{c}$ is the complementary factor of $\Delta$. Hence, in particular, if $\Delta$ is a two-sided inner function with values in $\mathcal{B}(E)$, then $\breve{\Delta}$ is of bounded type.

Proof. The first assertion follows from Lemma 4.5. The second assertion follows from the first assertion together with the observation that if $\Delta$ is two-sided inner then $\left[\Delta, \Delta_{c}\right]=\Delta$.

Corollary 4.21. Let $\Delta$ be an inner function with values in $\mathcal{B}(D, E)$. Then $[\Delta, \Omega]$ is two-sided inner for some inner function $\Omega$ with values in $\mathcal{B}\left(D^{\prime}, E\right)$ if and only if $\breve{\Delta}$ is of bounded type. 
Proof. Suppose that $[\Delta, \Omega]$ is two-sided inner for some inner function $\Omega$ with values in $\mathcal{B}\left(D^{\prime}, E\right)$. Then $\Delta^{*} \Omega=0$, so that $\Omega H_{D^{\prime}}^{2} \subseteq \operatorname{ker} \Delta^{*}=\Delta_{c} H_{D^{\prime \prime}}^{2}$. Thus $\Delta_{c}$ is a left inner divisor of $\Omega$, and hence $\left[\Delta, \Delta_{c}\right]$ is a left inner divisor of $[\Delta, \Omega]$. Therefore by Lemma 3.12, $\left[\Delta, \Delta_{c}\right]$ is two-sided inner, so that by Corollary 4.20 , $\breve{\Delta}$ is of bounded type. The converse follows at once from Corollary 4.20 with $\Omega=\Delta_{c}$.

We now ask: If $\Delta \equiv\left[\delta_{1}, \delta_{2}, \cdots, \delta_{m}\right] \in H_{M_{n \times m}}^{\infty}$ is an inner matrix function, does there exist $j(1 \leq j \leq m)$ such that $\operatorname{dc}\left\{\delta_{j}\right\}=\operatorname{dc}\{\Delta\}$ ? The answer, however, is negative. To see this, let $f$ and $g$ be given in Example 3.11 and let

$$
\Delta:=\left[\begin{array}{ll}
f & 0 \\
g & 0 \\
0 & f \\
0 & g
\end{array}\right] \equiv\left[\delta_{1}, \delta_{2}\right] .
$$

Since

$$
\left[\begin{array}{lll}
f & 0 & 0 \\
g & 0 & 0 \\
0 & 1 & 0 \\
0 & 0 & 1
\end{array}\right] \text { is inner, }
$$

in view of Corollary 4.16, we have $\mathrm{dc}\left(\delta_{1}\right) \leq 1$. But since $\mathrm{dc}\left(\delta_{1}\right) \neq 0$ (because $\delta_{1}^{*}$ is not of bounded type), it follows that $\operatorname{dc}\left\{\delta_{1}\right\}=1$. Similarly, $\operatorname{dc}\left\{\delta_{2}\right\}=1$. However, we have $\operatorname{dc}\{\Delta\}=2$, because we can show that $\Delta_{c}=0$.

\section{$\S$ 4.5. Meromorphic pseudo-continuations of bounded type}

In general, if a strong $L^{2}$-function $\Phi$ is of bounded type then we cannot guarantee that each entry $\phi_{i j} \equiv\left\langle\Phi d_{j}, e_{i}\right\rangle$ is of bounded type, where $\left\{d_{j}\right\}$ and $\left\{e_{i}\right\}$ are orthonormal bases of $D$ and $E$, respectively. But if we strengthen the assumption then we may have the assertion. To see this, for a function $\Psi: \mathbb{D}^{e} \equiv\{z: 1<|z| \leq$ $\infty\} \rightarrow \mathcal{B}(D, E)$, we define $\Psi_{\mathbb{D}}: \mathbb{D} \rightarrow \mathcal{B}(E, D)$ by

$$
\Psi_{\mathbb{D}}(\zeta):=\Psi^{*}(1 / \bar{\zeta}) \quad \text { for } \zeta \in \mathbb{D}
$$

If $\Psi_{\mathbb{D}}$ is a strong $H^{2}$-function, inner, and two-sided inner with values in $\mathcal{B}(E, D)$, then we shall say that $\Psi$ is a strong $H^{2}$-function, inner, and two-sided inner in $\mathbb{D}^{e}$ with values in $\mathcal{B}(D, E)$, respectively.

A $\mathcal{B}(D, E)$-valued function $\Psi$ is said to be meromorphic of bounded type in $\mathbb{D}^{e}$ if it can be represented by

$$
\Psi=\frac{G}{\theta},
$$

where $G$ is a strong $H^{2}$-function in $\mathbb{D}^{e}$, with values in $\mathcal{B}(D, E)$ and $\theta$ is a scalar inner function in $\mathbb{D}^{e}$. (cf. Fu2]). A function $\Phi \in L_{s}^{2}(\mathcal{B}(D, E)$ ) is said to have a meromorphic pseudo-continuation $\hat{\Phi}$ of bounded type in $\mathbb{D}^{e}$ if $\hat{\Phi}$ is meromorphic of bounded type in $\mathbb{D}^{e}$ and $\Phi$ is the nontangential SOT limit of $\hat{\Phi}$, that is, for all $x \in D$,

$$
\Phi(z) x=\hat{\Phi}(z) x:=\lim _{r z \rightarrow z} \hat{\Phi}(r z) x \quad \text { for almost all } z \in \mathbb{T} .
$$


Note that for almost all $z \in \mathbb{T}$,

$$
\Phi(z) x=\lim _{r z \rightarrow z} \hat{\Phi}(r z) x=\lim _{r z \rightarrow z} \hat{\Phi}_{\mathbb{D}}^{*}\left(r^{-1} z\right) x=\hat{\Phi}_{\mathbb{D}}^{*}(z) x \quad(x \in D) .
$$

We then have:

Lemma 4.22. Let $\Phi$ be a strong $L^{2}$-function with values in $\mathcal{B}(D, E)$. If $\Phi$ has a meromorphic pseudo-continuation of bounded type in $\mathbb{D}^{e}$, then $\breve{\Phi}$ is of bounded type.

Proof. Suppose that $\Phi$ has a meromorphic pseudo-continuation of bounded type in $\mathbb{D}^{e}$. Thus the meromorphic pseudo-continuation $\hat{\Phi}$ of $\Phi$ can be written as

$$
\hat{\Phi}(\zeta):=\frac{G(\zeta)}{\delta(\zeta)} \quad\left(\zeta \in \mathbb{D}^{e}\right)
$$

where $G$ is a strong $H^{2}$-function in $\mathbb{D}^{e}$, with values in $\mathcal{B}(D, E)$ and $\delta$ is a scalar inner function in $\mathbb{D}^{e}$. Then for all $x \in D$,

$$
\Phi(z) x=\hat{\Phi}_{\mathbb{D}}^{*}(z) x=\delta_{\mathbb{D}}(z) G_{\mathbb{D}}^{*}(z) x \quad \text { for almost all } z \in \mathbb{T} .
$$

Thus for all $x \in D, p \in \mathcal{P}_{E}$, and $n=1,2,3, \cdots$,

$$
\begin{aligned}
\int_{\mathbb{T}}\left\langle\Phi(z) x, z^{n} \delta_{\mathbb{D}}(z) p(z)\right\rangle_{E} d m(z) & =\int_{\mathbb{T}}\left\langle G_{\mathbb{D}}^{*}(z) x, z^{n} p(z)\right\rangle_{E} d m(z) \\
& =\left\langle x, z^{n} G_{\mathbb{D}}(z) p(z)\right\rangle_{L_{D}^{2}}=0,
\end{aligned}
$$

where the last equality follows from the fact that $z^{n} G_{\mathbb{D}}(z) p(z) \in z H_{D}^{2}$. Thus by Lemma 4.3. we can see that

$$
\delta_{\mathbb{D}} H_{E}^{2}=\operatorname{cl} \delta_{\mathbb{D}} \mathcal{P}_{E} \subseteq \operatorname{ker} H_{\Phi}^{*} .
$$

In view of Lemma 4.4, $\operatorname{ker} H_{\vec{\Phi}}^{*}=\Delta H_{E^{\prime}}^{2}$ for some inner function $\Delta$ with values in $\mathcal{B}\left(E^{\prime}, E\right)$. Thus $\Delta$ is a left inner divisor of $\delta_{\mathbb{D}} I_{E}$ (cf. $\mathbf{F F}, \mathbf{P e}$ ). Thus, it follows from Lemma 3.12 that that $\Delta$ is two-sided inner, so that $\breve{\Phi}$ is of bounded type.

The following lemma was proved in Fu1 under the more restrictive setting of $H^{\infty}(\mathcal{B}(D, E))$.

Lemma 4.23. Let $\Phi \in L^{\infty}(\mathcal{B}(D, E))$. Then the following are equivalent:

(a) $\Phi$ has a meromorphic pseudo-continuation of bounded type in $\mathbb{D}^{e}$;

(b) $\theta H_{E}^{2} \subseteq \operatorname{ker} H_{\Phi^{*}}$ for some scalar inner function $\theta$;

(c) $\Phi=\theta A^{*}$ for a scalar inner function $\theta$ and some $A \in H^{\infty}(\mathcal{B}(E, D))$.

Proof. First of all, recall that $L^{\infty}(\mathcal{B}(D, E)) \subseteq L_{s}^{2}(\mathcal{B}(D, E))$.

(a) $\Rightarrow$ (b): This follows from (4.15) in the proof of Lemma 4.22 .

(b) $\Rightarrow$ (c): Suppose that $\theta H_{E}^{2} \subseteq$ ker $H_{\Phi^{*}}$ for some scalar inner function $\theta$. Put $A:=\theta \Phi^{*}$. Then $A$ belongs to $H^{\infty}(\mathcal{B}(E, D))$ and $\Phi=\theta A^{*}$.

(c) $\Rightarrow$ (a): Suppose that $\Phi=\theta A^{*}$ for a scalar inner function $\theta$ and some $A \in H^{\infty}(\mathcal{B}(E, D))$. Thus it follows from Lemma 3.5 that $A$ is a strong $H^{2}$ function. Let

$$
\hat{\Phi}(\zeta):=\frac{A^{*}(1 / \bar{\zeta})}{\bar{\theta}(1 / \bar{\zeta})} \quad\left(\zeta \in \mathbb{D}^{e}\right)
$$


Then $\hat{\Phi}$ is meromorphic of bounded type in $\mathbb{D}^{e}$ and for all $x \in D$,

$$
\hat{\Phi}(z) x=\frac{A^{*}(z) x}{\bar{\theta}(z)}=\theta(z) A^{*}(z) x=\Phi(z) x \quad \text { for almost all } z \in \mathbb{T},
$$

which implies that $\Phi$ has a meromorphic pseudo-continuation of bounded type in $\mathbb{D}^{e}$.

An examination of the proof of Lemma 4.23 shows that Lemma 4.23 still holds for every function $\Phi \in L_{\mathcal{B}(D, E)}^{2}$.

Corollary 4.24. If $\Phi \in L_{\mathcal{B}(D, E)}^{2}$, then Lemma 4.23 holds with $A \in H_{\mathcal{B}(E, D)}^{2}$ in place of $A \in H^{\infty}(\mathcal{B}(E, D))$.

The following proposition gives an answer to an opening remark of this section.

Proposition 4.25. Let $D$ and $E$ be separable complex Hilbert spaces and let $\left\{d_{j}\right\}$ and $\left\{e_{i}\right\}$ be orthonormal bases of $D$ and $E$, respectively. If $\Phi \in L_{\mathcal{B}(D, E)}^{2}$ has a meromorphic pseudo-continuation of bounded type in $\mathbb{D}^{e}$, then $\breve{\phi}_{i j}(z) \equiv$ $\left\langle\breve{\Phi}(z) d_{j}, e_{i}\right\rangle_{E}$ is of bounded type for each $i, j$.

Proof. Let $\Phi \in L_{\mathcal{B}(D, E)}^{2}$. Suppose that $\Phi$ has a meromorphic pseudocontinuation of bounded type in $\mathbb{D}^{e}$. Then by Corollary 4.24, $\Phi=\theta A^{*}$ for a scalar inner function $\theta$ and some $A \in H_{\mathcal{B}(E, D)}^{2}$. Write

$$
\phi_{i j}(z):=\left\langle\Phi(z) d_{j}, e_{i}\right\rangle_{E} \quad \text { and } \quad a_{i j}(z):=\left\langle\widetilde{A}(z) d_{j}, e_{i}\right\rangle_{E} .
$$

Then for each $i, j$,

$$
\begin{aligned}
\int_{\mathbb{T}}\left|\phi_{i j}(z)\right|^{2} d m(z) & =\int_{\mathbb{T}}\left|\left\langle\Phi(z) d_{j}, e_{i}\right\rangle_{E}\right|^{2} d m(z) \\
& \leq \int_{\mathbb{T}}|| \Phi(z) \|_{\mathcal{B}(D, E)}^{2} d m(z)<\infty,
\end{aligned}
$$

which implies $\phi_{i j} \in L^{2}$. Similarly, $a_{i j} \in L^{2}$ and for $n=1,2,3, \cdots$,

$$
\widehat{a_{i j}}(-n)=\int_{\mathbb{T}} z^{n}\left\langle\widetilde{A}(z) d_{j}, e_{i}\right\rangle_{E} d m(z)=\left\langle d_{j}, z^{-n} \breve{A}(z) e_{i}\right\rangle_{L_{D}^{2}}=0,
$$

which implies $a_{i j} \in H^{2}$. Note that

$$
\breve{\phi}_{i j}(z)=\breve{\theta}(z)\left\langle\widetilde{A}(z) d_{j}, e_{i}\right\rangle_{E}=\breve{\theta}(z) a_{i j}(z),
$$

which implies that $\breve{\phi}_{i j}$ is of bounded type for each $i, j$.

EXAmPLE 4.26. The converse of Lemma 4.22 is not true in general. To see this, let $\left\{\alpha_{n}\right\}$ be a sequence of distinct points in $\mathbb{D}$ such that $\sum_{n=1}^{\infty}\left(1-\left|\alpha_{n}\right|\right)=\infty$ and put $\Delta:=\operatorname{diag}\left(b_{\alpha_{n}}\right)$, where $b_{\alpha_{n}}(z):=\frac{z-\alpha_{n}}{1-\overline{\alpha_{n}} z}$. Then $\Delta$ is two-sided inner, and hence by Lemma 4.20, $\breve{\Delta}$ is of bounded type. On the other hand, by Lemma 4.5 ker $H_{\Delta^{*}}=\Delta H_{\ell^{2}}^{2}$. Thus if $\Delta$ had a meromorphic pseudo-continuation of bounded type in $\mathbb{D}^{e}$, then by Lemma 4.23, we would have $\theta H_{\ell^{2}}^{2} \subseteq \Delta H_{\ell^{2}}^{2}$ for a scalar inner function $\theta$, so that we should have $\theta\left(\alpha_{n}\right)=0$ for each $n=1,2, \cdots$, and hence $\theta=0$, a contradiction. Therefore, $\Delta$ cannot have a meromorphic pseudo-continuation of bounded type in $\mathbb{D}^{e}$. 
For matrix-valued cases, a function having a meromorphic pseudo-continuation of bounded type in $\mathbb{D}^{e}$ is actually a function whose flip is of bounded type.

Corollary 4.27. For $\Phi \equiv\left[\phi_{i j}\right] \in L_{M_{n \times m}}^{2}$, the following are equivalent:

(a) $\Phi$ has a meromorphic pseudo-continuation of bounded type in $\mathbb{D}^{e}$;

(b) $\breve{\Phi}$ is of bounded type;

(c) $\breve{\phi}_{i j}$ is of bounded type for each $i, j$.

Proof. (a) $\Rightarrow(\mathrm{b})$ : This follows from Lemma 4.22 .

$(\mathrm{b}) \Rightarrow(\mathrm{a})$ : Suppose that $\breve{\Phi}$ is of bounded type. Then $\operatorname{ker} H_{\breve{\Phi}}^{*}=\Theta H_{\mathbb{C}^{n}}^{2}$ for some two-sided inner function $\Theta \in H_{M_{n}}^{\infty}$. Thus by the Complementing Lemma (cf. p. 29), there exist a scalar inner function $\theta$ and a function $G$ in $H_{M_{n}}^{\infty}$ such that $G \Theta=\Theta G=\theta I_{n}$, and hence, $\theta H_{\mathbb{C}^{n}}^{2}=\Theta G H_{\mathbb{C}^{n}}^{2} \subseteq \Theta H_{\mathbb{C}^{n}}^{2}=\operatorname{ker} H_{\breve{\Phi}}^{*}$. It thus follows from Corollary 4.24 that $\breve{\Phi}$ has a meromorphic pseudo-continuation of bounded type in $\mathbb{D}^{e}$.

(a) $\Leftrightarrow(\mathrm{c})$ : This follows from Corollary 4.24 and Proposition 4.25 .

However, by contrast to the matrix-valued case, it may happened that an $L^{\infty}$ function $\Phi$ is not of bounded type in the sense of Definition 4.18 even though each entry $\phi_{i j}$ of $\Phi$ is of bounded type.

EXAMPLE 4.28. Let $\left\{\alpha_{j}\right\}$ be a sequence of distinct points in $(0,1)$ satisfying $\sum_{j=1}^{\infty}\left(1-\alpha_{j}\right)<\infty$. For each $j \in \mathbb{Z}_{+}$, choose a sequence $\left\{\alpha_{i j}\right\}$ of distinct points on the circle $C_{j}:=\left\{z \in \mathbb{C}:|z|=\alpha_{j}\right\}$. Let

$$
B_{i j}:=\frac{\bar{b}_{\alpha_{i j}}}{(i+j) !} \quad\left(i, j \in \mathbb{Z}_{+}\right),
$$

where $b_{\alpha}(z):=\frac{z-\alpha}{1-\bar{\alpha} z}$, and let

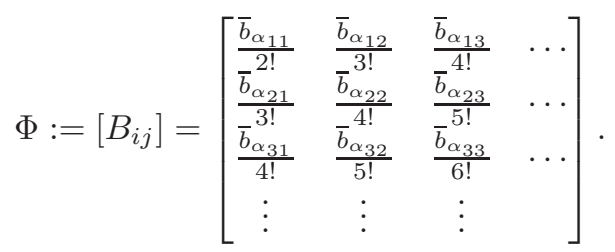

Observe that

$$
\sum_{i, j}\left|B_{i j}(z)\right|^{2}=\sum_{i} \frac{i}{((1+i) !)^{2}} \leq \sum_{i} \frac{1}{(1+i)^{2}}<\infty
$$

which implies that $\Phi \in L^{\infty}\left(\mathcal{B}\left(\ell^{2}\right)\right)$. For a function $f \in H_{\ell^{2}}^{2}$, we write $f=$ $\left(f_{1}, f_{2}, f_{3}, \cdots\right)^{t}\left(f_{n} \in H^{2}\right)$. Thus if $f=\left(f_{1}, f_{2}, f_{3}, \cdots\right)^{t} \in \operatorname{ker} H_{\Phi}$, then $\sum_{j} \frac{\bar{b}_{\alpha_{i j}}}{(i+j) !} f_{j} \in$ $H^{2}$ for each $i \in \mathbb{Z}_{+}$, which forces that $f_{j}\left(\alpha_{i j}\right)=0$ for each $i, j$. Thus $f_{j}=0$ for each $j$ (by the Identity Theorem). Therefore we can conclude that ker $H_{\widetilde{\Phi}}^{*}=\{0\}$, so that $\widetilde{\Phi}$ is not of bounded type. But we note that every entry of $\widetilde{\Phi}$ is of bounded type.

We conclude this chapter with an application to $C_{0}$-contractions.

The class $C_{0}$. denotes the set of all contractions $T \in \mathcal{B}(\mathcal{H})$ satisfying the condition (1.4). The class $C_{00}$ denotes the set of all contractions $T \in \mathcal{B}(\mathcal{H})$ such 
that $\lim _{n \rightarrow \infty} T^{n} x=0$ and $\lim _{n \rightarrow \infty} T^{* n} x=0$ for each $x \in H$. It was known ([Ni1, p.43]) that if $T$ is a $C_{0}$--contraction with characteristic function $\Delta$ (i.e., $\left.\left.T \cong S_{E}^{*}\right|_{\mathcal{H}(\Delta)}\right)$, then

$$
T \in C_{00} \Longleftrightarrow \Delta \text { is two-sided inner. }
$$

A contraction $T \in \mathcal{B}(\mathcal{H})$ is called a completely non-unitary (c.n.u.) if there exists no nontrivial reducing subspace on which $T$ is unitary. The class $C_{0}$ is the set of all c.n.u. contractions $T$ such that there exists a nonzero function $\varphi \in H^{\infty}$ annihilating $T$, i.e., $\varphi(T)=0$, where $\varphi(T)$ is given by the calculus of Sz.-Nagy and Foiaş. We can easily check that $C_{0} \subseteq C_{00}$. Moreover, it is well known (Ni1, p.73]) that if $T:=\left.P_{\mathcal{H}(\Delta)} S_{E}\right|_{\mathcal{H}(\Delta)} \in C_{00}$ and $\varphi \in H^{\infty}$, then

$$
\varphi(T)=0 \Longleftrightarrow \exists G \in H^{\infty}(\mathcal{B}(E)) \text { such that } G \Delta=\Delta G=\varphi I_{E} .
$$

The theory of spectral multiplicity for operators of class $C_{0}$ has been well developed (see [Ni1, Appendix 1], SFBK]). If $T \in C_{0}$, then there exists an inner function $m_{T}$ such that $m_{T}(T)=0$ and

$$
\varphi \in H^{\infty}, \varphi(T)=0 \Longrightarrow \varphi / m_{T} \in H^{\infty} .
$$

The function $m_{T}$ is called the minimal annihilator of the operator $T$.

In view of (4.16), we may ask what is a condition on the characteristic function $\Delta$ of $T$ for a $C_{0}$.-contraction $T$ to belong to the class $C_{0}$. The following proposition gives an answer.

Proposition 4.29. Let $T:=\left.S_{E}^{*}\right|_{\mathcal{H}(\Delta)}$ for an inner function $\Delta$ with values in $\mathcal{B}(D, E)$. Then the following are equivalent:

(a) $T \in C_{0}$;

(b) $\Delta$ is two-sided inner and has a meromorphic pseudo-continuation of bounded type in $\mathbb{D}^{e}$.

Hence, in particular, if $\Delta$ is an inner matrix function then $T \in C_{0}$ if and only if $T \in C_{00}$.

Proof. (a) $\Rightarrow$ (b): Suppose $T \in C_{0}$, and hence $\varphi(T)=0$ for some nonzero function $\varphi \in H^{\infty}$. Then $T \in C_{00}$, so that by the above remark, $\Delta$ is two-sided inner. Thus by the Model Theorem, we have

$$
\left.T \cong P_{\mathcal{H}(\widetilde{\Delta})} S_{E}\right|_{\mathcal{H}(\widetilde{\Delta})}
$$

It thus follows from (4.17) that there exists $\Omega \in H^{\infty}(\mathcal{B}(E))$ such that $\widetilde{\Delta} \Omega=\Omega \widetilde{\Delta}=$ $\varphi I_{E}$. Thus $H_{\Delta^{*}}\left(\widetilde{\varphi} H_{E}^{2}\right)=H_{\Delta^{*}}\left(\Delta \widetilde{\Omega} H_{E}^{2}\right)=0$. We thus have

$$
\widetilde{\varphi}^{i} H_{E}^{2} \subseteq \operatorname{cl} \widetilde{\varphi} H_{E}^{2} \subseteq \operatorname{ker} H_{\Delta^{*}} .
$$

It thus follows from Lemma 4.23 that $\Delta$ has a meromorphic pseudo-continuation of bounded type in $\mathbb{D}^{e}$. This gives the implication $(\mathrm{a}) \Rightarrow(\mathrm{b})$.

(b) $\Rightarrow$ (a): Suppose that $\Delta$ is two-sided inner and has a meromorphic pseudocontinuation of bounded type in $\mathbb{D}^{e}$. Then by Lemma 4.5 and Lemma 4.23, there exists a scalar function $\delta$ such that $\delta H_{E}^{2} \subseteq \operatorname{ker} H_{\Delta^{*}}=\Delta H_{E}^{2}$. Thus we may write $\delta I_{E}=\Delta \Omega=\Omega \Delta$ for some $\Omega \in H^{\infty}(\mathcal{B}(E))$. Thus we have

$$
\delta\left(\left.P_{\mathcal{H}(\Delta)} S_{E}\right|_{\mathcal{H}(\Delta)}\right)=\left.P_{\mathcal{H}(\Delta)}\left(\delta I_{E}\right)\right|_{\mathcal{H}(\Delta)}=0,
$$


so that

$$
\widetilde{\delta}(T)=\left(\delta\left(T^{*}\right)\right)^{*}=\left(\delta\left(\left.P_{\mathcal{H}(\Delta)} S_{E}\right|_{\mathcal{H}(\Delta)}\right)\right)^{*}=0,
$$

which gives $T \in C_{0}$. This prove the implication $(\mathrm{b}) \Rightarrow(\mathrm{a})$.

The second assertion follows from the first together with Corollary 4.20 and Corollary 4.27 . 



\section{CHAPTER 5}

\section{A canonical decomposition of strong $L^{2}$-functions}

In this chapter, we establish a canonical decomposition of strong $L^{2}$-functions. To better understand this canonical decomposition, we first consider an example of a matrix-valued $L^{2}$-function that does not admit a Douglas-Shapiro-Shields factorization. Suppose that $\theta_{1}$ and $\theta_{2}$ are coprime inner functions. Consider

$$
\Phi:=\left[\begin{array}{ccc}
\theta_{1} & 0 & 0 \\
0 & \theta_{2} & 0 \\
0 & 0 & a
\end{array}\right] \equiv\left[\phi_{1}, \phi_{2}, \phi_{3}\right] \in H_{M_{3}}^{\infty},
$$

where $a \in H^{\infty}$ is such that $\bar{a}$ is not of bounded type. Then a direct calculation shows that

$$
\operatorname{ker} H_{\Phi^{*}}=\left[\begin{array}{cc}
\theta_{1} & 0 \\
0 & \theta_{2} \\
0 & 0
\end{array}\right] H_{\mathbb{C}^{2}}^{2} \equiv \Delta H_{\mathbb{C}^{2}}^{2} .
$$

Since $\Delta$ is not two-sided inner, it follows from Lemma 4.2 that $\Phi$ does not admit a Douglas-Shapiro-Shields factorization. For a decomposition of $\Phi$, suppose that

$$
\Phi=\Omega A^{*},
$$

where $\Omega, A \in H_{M_{3 \times k}}^{2}(k=1,2), \Omega$ is an inner function, and $\Omega$ and $A$ are right coprime. We then have

$$
\Phi^{*} \Omega=A \in H_{M_{3 \times k}}^{2} .
$$

But since $\bar{a}$ is not of bounded type, it follows from (5.2) that the 3 rd row vector of $\Omega$ is zero. Thus by (5.1), we must have $a=0$, a contradiction. Therefore we could not get any decomposition of the form $\Phi=\Omega A^{*}$ with a $3 \times k$ inner matrix function $\Omega$ for each $k=1,2,3$. To get another idea, we note that ker $\Delta^{*}=\left[\begin{array}{lll}0 & 0 & 1\end{array}\right]^{t} H^{2} \equiv \Delta_{c} H^{2}$. Then by a direct manipulation, we can get

$$
\Phi=\left[\begin{array}{ccc}
\theta_{1} & 0 & 0 \\
0 & \theta_{2} & 0 \\
0 & 0 & a
\end{array}\right]=\left[\begin{array}{cc}
\theta_{1} & 0 \\
0 & \theta_{2} \\
0 & 0
\end{array}\right]\left[\begin{array}{ll}
1 & 0 \\
0 & 1 \\
0 & 0
\end{array}\right]^{*}+\left[\begin{array}{l}
0 \\
0 \\
1
\end{array}\right]\left[\begin{array}{lll}
0 & 0 & a
\end{array}\right] \equiv \Delta A^{*}+\Delta_{c} C
$$

where $\Delta$ and $A$ are right coprime because $\widetilde{\Delta} H_{\mathbb{C}^{3}}^{2} \bigvee \widetilde{A} H_{\mathbb{C}^{3}}^{2}=H_{\mathbb{C}^{2}}^{2}$.

To encounter another situation, consider

$$
\Phi:=\left[\begin{array}{ccc}
f & f & 0 \\
g & g & 0 \\
0 & 0 & \theta \bar{a}
\end{array}\right] \equiv\left[\phi_{1}, \phi_{2}, \phi_{3}\right] \in H_{M_{3}}^{\infty},
$$


where $f$ and $g$ are given in Example 3.11. $\theta$ is inner, and $a \in H^{\infty}$ is such that $\theta$ and $a$ are coprime. It then follows from Lemma 4.5 that

$$
\operatorname{ker} H_{[\bar{f} \bar{g}]}=\left[\begin{array}{l}
f \\
g
\end{array}\right] H^{2}
$$

We thus have that

$$
\operatorname{ker} H_{\Phi^{*}}=\operatorname{ker} H_{[\bar{f} \bar{g}]} \bigoplus \operatorname{ker} H_{\bar{\theta} a}=\left[\begin{array}{ll}
f & 0 \\
g & 0 \\
0 & \theta
\end{array}\right] H_{\mathbb{C}^{2}}^{2} \equiv \Delta H_{\mathbb{C}^{2}}^{2} .
$$

Thus by Lemma 4.2. $\Phi$ does not admit a Douglas-Shapiro-Shields factorization. Observe that

$$
\Phi=\left[\begin{array}{ccc}
f & f & 0 \\
g & g & 0 \\
0 & 0 & \theta \bar{a}
\end{array}\right]=\left[\begin{array}{ll}
f & 0 \\
g & 0 \\
0 & \theta
\end{array}\right]\left[\begin{array}{ll}
1 & 0 \\
1 & 0 \\
0 & a
\end{array}\right]^{*}=\Delta A^{*}
$$

Since $\widetilde{\theta}$ and $\widetilde{a}$ are coprime, it follows that $\Delta$ and $A$ are right coprime. Note that $\Delta$ is not two-sided inner and ker $\Delta^{*}=\{0\}$.

The above examples (5.3) and (5.4) seem to signal that the decomposition of a matrix-valued $H^{2}$-functions $\Phi$ satisfying ker $H_{\breve{\Phi}}^{*}=\Delta H_{\mathbb{C}^{n}}^{2}$ may be affected by the kernel of $\Delta^{*}$ and in turn, the complementary factor $\Delta_{c}$ of $\Delta$. Indeed, if we regard $\Delta^{*}$ as an operator acting from $L_{E}^{2}$, and hence ker $\Delta^{*} \subseteq L_{E}^{2}$, then $B$ in the canonical decomposition (5.5) satisfies the inclusion $\{B\} \subseteq$ ker $\Delta^{*}$. The following theorem gives a canonical decomposition of strong $L^{2}$-functions which realizes the idea inside those examples.

We are ready for:

ThEOREM 5.1. (A canonical decomposition of strong $L^{2}$-functions) If $\Phi$ is a strong $L^{2}$-function with values in $\mathcal{B}(D, E)$, then $\Phi$ can be expressed in the form

$$
\Phi=\Delta A^{*}+B
$$

where

(i) $\Delta$ is an inner function with values in $\mathcal{B}\left(E^{\prime}, E\right), \widetilde{A} \in H_{s}^{2}\left(\mathcal{B}\left(D, E^{\prime}\right)\right)$, and $B \in L_{s}^{2}(\mathcal{B}(D, E))$;

(ii) $\Delta$ and $A$ are right coprime;

(iii) $\Delta^{*} B=0$;

(iv) $\operatorname{nc}\left\{\Phi_{+}\right\} \leq \operatorname{dim} E^{\prime}$.

In particular, if $\operatorname{dim} E^{\prime}<\infty$ (for instamce, $\operatorname{dim} E<\infty$ ), then the expression (5.5) is unique (up to a unitary constant right factor).

Proof. If ker $H_{\breve{\Phi}}^{*}=\{0\}$, take $E^{\prime}:=\{0\}$ and $B:=\Phi$. Then $\widetilde{\Delta}$ and $\widetilde{A}$ are zero operator with codomain $\{0\}$. Thus $\Phi=\Delta A^{*}+B$, where $\Delta$ and $A$ are right coprime. It also follows from Theorem 4.10 that $\operatorname{nc}\left\{\Phi_{+}\right\}=0$, which gives the inequality (iv). If instead ker $H_{\breve{\Phi}}^{*} \neq\{0\}$, then in view of Lemma 4.4, we may suppose ker $H_{\breve{\Phi}}^{*}=\Delta H_{E^{\prime}}^{2}$ for some nonzero inner function $\Delta$ with values in $\mathcal{B}\left(E^{\prime}, E\right)$. Put $A:=\Phi^{*} \Delta$. Then it follows from Lemma 3.14 that $A^{*}$ is a strong $L^{2}$-function 
with values in $\mathcal{B}\left(D, E^{\prime}\right)$. Thus $\widetilde{A}=\breve{A}^{*}$ is a strong $L^{2}$-function with values in $\mathcal{B}\left(D, E^{\prime}\right)$. Since ker $H_{\stackrel{\Phi}{*}}^{*}=\Delta H_{E^{\prime}}^{2}$, it follows that for all $p \in \mathcal{P}_{D}$ and $h \in H_{E^{\prime}}^{2}$

$$
\begin{aligned}
0 & =\left\langle H_{\breve{\Phi}} p, \Delta h\right\rangle_{L_{E}^{2}} \\
& =\int_{\mathbb{T}}\langle\breve{\Phi}(z) p(z), \bar{z} \Delta(\bar{z}) h(\bar{z})\rangle_{E} d m(z) \\
& =\int_{\mathbb{T}}\langle\widetilde{\Delta}(z) \breve{\Phi}(z) p(z), \bar{z} h(\bar{z})\rangle_{E^{\prime}} d m(z) \\
& =\left\langle H_{\widetilde{A}} p, h\right\rangle_{L_{E^{\prime}}^{2}},
\end{aligned}
$$

which implies $H_{\widetilde{A}}=0$. Thus by Lemma 4.1 $\widetilde{A}$ belongs to $H_{s}^{2}(\mathcal{B}(D, E))$. Put $B:=\Phi-\Delta A^{*}$. Then by Lemma 3.14, $B$ is a strong $L^{2}$-function with values in $\mathcal{B}(D, E)$. Observe that

$$
\Phi=\Delta A^{*}+B \text { and } \quad \Delta^{*} B=0 .
$$

For the first assertion, we need to show that $\Delta$ and $A$ are right coprime. To see this, we suppose that $\Omega$ is a common left inner divisor, with values in $\mathcal{B}\left(E^{\prime \prime}, E^{\prime}\right)$, of $\widetilde{\Delta}$ and $\widetilde{A}$. Then we may write

$$
\widetilde{\Delta}=\Omega \widetilde{\Delta}_{1} \quad \text { and } \quad \widetilde{A}=\Omega \widetilde{A}_{1},
$$

where $\widetilde{\Delta}_{1} \in H^{\infty}\left(\mathcal{B}\left(E, E^{\prime \prime}\right)\right)$ and $\widetilde{A}_{1} \in H_{s}^{2}\left(\mathcal{B}\left(D, E^{\prime \prime}\right)\right)$. Thus we have

$$
\Delta=\Delta_{1} \widetilde{\Omega} \text { and } A=A_{1} \widetilde{\Omega} .
$$

Since $\Omega$ is inner, it follows that $\Delta_{1}=\Delta \widetilde{\Omega}^{*}$, and hence, by Lemma 3.13, $\Delta_{1}$ is inner. We now claim that

$$
\Delta_{1} H_{E^{\prime \prime}}^{2}=\operatorname{ker} H_{\overparen{\Phi}}^{*}=\Delta H_{E^{\prime}}^{2}
$$

Since $\Omega$ is an inner function with values in $\mathcal{B}\left(E^{\prime \prime}, E^{\prime}\right)$, we know that $\widetilde{\Omega} \in H^{\infty}\left(\mathcal{B}\left(E^{\prime}, E^{\prime \prime}\right)\right)$ by Lemma 3.13. Thus it follows from Corollary 3.15 and (5.6) that

$$
\Delta H_{E^{\prime}}^{2}=\Delta_{1} \widetilde{\Omega} H_{E^{\prime}}^{2} \subseteq \Delta_{1} H_{E^{\prime \prime}}^{2} .
$$

For the reverse inclusion, by (5.6), we may write $\Phi=\Delta_{1} A_{1}^{*}+B$. Since $0=$ $\Delta^{*} B=\widetilde{\Omega}^{*} \Delta_{1}^{*} B$, it follows that $\Delta_{1}^{*} B=0$. Therefore for all $f \in H_{E^{\prime \prime}}^{2}, x \in D$ and $n=1,2, \cdots$, we have

$$
\begin{aligned}
\int_{\mathbb{T}}\left\langle\Phi(z) x, z^{n} \Delta_{1}(z) f(z)\right\rangle_{E} d m(z) & =\int_{\mathbb{T}}\left\langle\left(\Delta_{1}(z) A_{1}^{*}(z)+B(z)\right) x, z^{n} \Delta_{1}(z) f(z)\right\rangle_{E} d m(z) \\
& =\int_{\mathbb{T}}\left\langle A_{1}^{*}(z) x, z^{n} f(z)\right\rangle_{E^{\prime \prime}} d m(z) \\
& =\left\langle A_{1}^{*}(z) x, z^{n} f(z)\right\rangle_{L_{E^{\prime \prime}}^{2}} \\
& =0,
\end{aligned}
$$

where the last equality follows from the fact that $A_{1}^{*}(z) x=\widetilde{A}_{1}(\bar{z}) x \in L_{E^{\prime \prime}}^{2} \ominus z H_{E^{\prime \prime}}^{2}$. Thus by Lemma 4.3 , we have

$$
\Delta_{1} H_{E^{\prime \prime}}^{2} \subseteq \operatorname{ker} H_{\overleftarrow{\Phi}}^{*}=\Delta H_{E^{\prime}}^{2},
$$

which proves (5.7). Thus it follows from the Beurling-Lax-Halmos Theorem and (5.6) that $\widetilde{\Omega}$ is a unitary operator, and so is $\Omega$. Therefore $A$ and $\Delta$ are right 
coprime. The assertion (iv) on the nc-number comes from Theorem 4.10. This proves the first assertion (5.5).

Suppose $\operatorname{dim} E^{\prime}<\infty$. For the uniqueness of the expression (5.5), we suppose that $\Phi=\Delta_{1} A_{1}^{*}+B_{1}=\Delta_{2} A_{2}^{*}+B_{2}$ are two canonical decompositions of $\Phi$. We want to show that $\Delta_{1}=\Delta_{2}$, which gives

$$
A_{1}^{*}=\Delta_{1}^{*}\left(\Delta_{1} A_{1}^{*}+B_{1}\right)=\Delta_{2}^{*}\left(\Delta_{2} A_{2}^{*}+B_{2}\right)=A_{2}^{*}
$$

and in turn, $B_{1}=B_{2}$, which implies that the representation (5.5) is unique. To prove $\Delta_{1}=\Delta_{2}$, it suffices to show that if $\Phi=\Delta A^{*}+B$ is a canonical decomposition of $\Phi$, then

$$
\operatorname{ker} H_{\overleftrightarrow{\Phi}}^{*}=\Delta H_{E^{\prime}}^{2}
$$

If $E^{\prime}=\{0\}$, then $\operatorname{nc}\left\{\Phi_{+}\right\}=0$. Thus it follows from Corollary 4.11 that

$$
\operatorname{ker} H_{\Phi}^{*}=\{0\}=\Delta H_{E^{\prime}}^{2},
$$

which proves (5.8). If instead $E^{\prime} \neq\{0\}$, then we suppose $r:=\operatorname{dim} E^{\prime}<\infty$. Thus, we may assume that $E^{\prime}=\mathbb{C}^{r}$, so that $\Delta$ is an inner function with values in $\mathcal{B}\left(\mathbb{C}^{r}, E\right)$. Suppose that $\Phi=\Delta A^{*}+B$ is a canonical decomposition of $\Phi$ in $L_{s}^{2}(\mathcal{B}(D, E))$. We first claim that

$$
\Delta H_{\mathbb{C}^{r}}^{2} \subseteq \operatorname{ker} H_{\overparen{\Phi}}^{*} .
$$

Observe that for each $g \in H_{\mathbb{C}^{r}}^{2}, x \in D$ and $k=1,2,3, \cdots$,

$$
\begin{aligned}
\int_{\mathbb{T}}\left\langle\Phi(z) x, z^{k} \Delta(z) g(z)\right\rangle_{E} d m(z) & =\int_{\mathbb{T}}\left\langle A^{*}(z) x, z^{k} g(z)\right\rangle_{\mathbb{C}^{r}} d m(z) \\
& =\left\langle\widetilde{A}(\bar{z}) x, z^{k} g(z)\right\rangle_{L_{\mathbb{C}^{r}}^{2}} \\
& =0 .
\end{aligned}
$$

It thus follows from Lemma 4.3 that $\Delta H_{\mathbb{C}^{r}}^{2} \subseteq \operatorname{ker} H_{\overparen{\Phi}}^{*}$, which proves (5.9). In view of Lemma 4.4 we may assume that $\operatorname{ker} H_{\stackrel{\Phi}{*}}^{*}=\Theta H_{E^{\prime \prime}}^{2}$ for some inner function $\Theta$ with values in $\mathcal{B}\left(E^{\prime \prime}, E\right)$. Then by Theorem 4.10

$$
p \equiv \operatorname{dim} E^{\prime \prime}=\mathrm{nc}\left\{\Phi_{+}\right\} \leq r .
$$

Thus we may assume $E^{\prime \prime} \equiv \mathbb{C}^{p}$. Since

$$
\Delta H_{\mathbb{C}^{r}}^{2} \subseteq \operatorname{ker} H_{\overparen{\Phi}}^{*}=\Theta H_{\mathbb{C}^{p}}^{2},
$$

it follows that $\Theta$ is left inner divisor of $\Delta$, i.e., there exists a $p \times r$ inner matrix function $\Delta_{1}$ such that $\Delta=\Theta \Delta_{1}$. Since $\Delta_{1}$ is inner, it follows that $r \leq p$. But since by (5.10), $p \leq r$, we must have $r=p$, which implies that $\Delta_{1}$ is two-sided inner. Thus we have

$$
\Theta^{*} \Phi=\Delta_{1} A^{*}+\Delta_{1} \Delta^{*} B=\Delta_{1} A^{*} .
$$

Since ker $H_{\overparen{\Phi}}^{*}=\Theta H_{\mathbb{C}^{r}}^{2}$, it follows from Lemma 4.3 and (5.12) that for all $f \in H_{\mathbb{C}^{r}}^{2}$, $x \in D$ and $n=1,2, \cdots$,

$$
\int_{\mathbb{T}}\left\langle\Delta_{1}(z) A^{*}(z) x, z^{n} f(z)\right\rangle_{\mathbb{C}^{r}} d m(z)=\int_{\mathbb{T}}\left\langle\Phi(z) x, z^{n} \Theta(z) f(z)\right\rangle_{E} d m(z)=0 .
$$

Write $\Psi:=\Delta_{1} A^{*}$. Then by Lemma 3.14 $\Psi \in L_{s}^{2}\left(\mathcal{B}\left(D, \mathbb{C}^{r}\right)\right)$. Thus by Lemma 4.1. Lemma 4.3 and (5.13), we have $\breve{\Psi} \in H_{s}^{2}\left(\mathcal{B}\left(D, \mathbb{C}^{r}\right)\right)$. Since $\widetilde{A}=\widetilde{\Delta}_{1} \breve{\Psi}$, it follows that $\widetilde{\Delta}_{1}$ is a common left inner divisor of $\widetilde{\Delta}$ and $\widetilde{A}$. But since $\Delta$ and $A$ are right 
coprime, it follows that $\widetilde{\Delta}_{1}$ is a unitary matrix, and so is $\Delta_{1}$, which proves (5.8). This proves the uniqueness of the expression (5.5) when $\operatorname{dim} E^{\prime}<\infty$.

This completes the proof.

The proof of Theorem 5.1 shows that the inner function $\Delta$ in a canonical decomposition (5.5) of a strong $L^{2}$-function $\Phi$ can be obtained from equation

$$
\operatorname{ker} H_{\breve{\Phi}}^{*}=\Delta H_{E^{\prime}}^{2}
$$

which is guaranteed by the Beurling-Lax-Halmos Theorem (see Corollary 4.4). In this case, the expression (5.5) will be called the BLH-canonical decomposition of $\Phi$ in the viewpoint that $\Delta$ comes from the Beurling-Lax-Halmos Theorem. However, if $\operatorname{dim} E^{\prime}=\infty$ (even though $\operatorname{dim} D<\infty$ ), then it is possible to get another inner function $\Theta$ of a canonical decomposition (5.5) for the same function: in this case, ker $H_{\overparen{\Phi}}^{*} \neq \Theta H_{E^{\prime \prime}}^{2}$. Indeed, the following remark shows that the canonical decomposition (5.5) is not unique in general.

Remark 5.2. If $\operatorname{dim} E^{\prime}=\infty$ (even though $\operatorname{dim} D<\infty$ ), the canonical decomposition (5.5) may not be unique even if $\breve{\Phi}$ is of bounded type. To see this, let $\Phi$ be an inner function with values in $\mathcal{B}\left(\mathbb{C}^{2}, \ell^{2}\right)$ defined by

$$
\Phi:=\left[\begin{array}{cc}
\theta_{1} & 0 \\
0 & 0 \\
0 & \theta_{2} \\
0 & 0 \\
0 & 0 \\
0 & 0 \\
\vdots & \vdots
\end{array}\right]
$$

where $\theta_{1}$ and $\theta_{2}$ are scalar inner functions. Then

$$
\operatorname{ker} H_{\Phi}^{*}=\operatorname{ker} H_{\Phi^{*}}=\operatorname{diag}\left(\theta_{1}, 1, \theta_{2}, 1,1,1, \cdots\right) H_{\ell^{2}}^{2} \equiv \Theta H_{\ell^{2}}^{2},
$$

which implies that $\breve{\Phi}$ is of bounded type since $\Theta$ is two-sided inner (see Definition 4.18). Let

$$
A:=\Phi^{*} \Theta=\left[\begin{array}{cccccccc}
1 & 0 & 0 & 0 & 0 & 0 & 0 & \cdots \\
0 & 0 & 1 & 0 & 0 & 0 & 0 & \cdots
\end{array}\right] \text { and } B:=0
$$

Then $\widetilde{A}$ belongs to belongs to $H_{s}^{2}\left(\mathcal{B}\left(\mathbb{C}^{2}, \ell^{2}\right)\right)$ and $\widetilde{\Theta} H_{\ell^{2}}^{2} \bigvee \widetilde{A} H_{\mathbb{C}^{2}}^{2}=H_{\ell^{2}}^{2}$, which implies that $\Theta$ and $A$ are right coprime. Clearly, $\Theta^{*} B=0$ and $\operatorname{nc}\left\{\Phi_{+}\right\} \leq \operatorname{dim} \ell^{2}=\infty$. Therefore,

$$
\Phi=\Theta A^{*}
$$


is the BLH-canonical decomposition of $\Phi$. On the other hand, to get another canonical decomposition of $\Phi$, let

$$
\Delta:=\left[\begin{array}{cccccccc}
\theta_{1} & 0 & 0 & 0 & 0 & 0 & 0 & \cdots \\
0 & 0 & 0 & 0 & 0 & 0 & 0 & \cdots \\
0 & \theta_{2} & 0 & 0 & 0 & 0 & 0 & \cdots \\
0 & 0 & 1 & 0 & 0 & 0 & 0 & \cdots \\
0 & 0 & 0 & 1 & 0 & 0 & 0 & \cdots \\
0 & 0 & 0 & 0 & 1 & 0 & 0 & \cdots \\
\vdots & \vdots & \vdots & \vdots & \vdots & \vdots & \vdots & \ddots
\end{array}\right] .
$$

Then $\Delta$ is an inner function. If we define

$$
A_{1}:=\left[\begin{array}{cccccccc}
1 & 0 & 0 & 0 & 0 & 0 & 0 & \cdots \\
0 & 1 & 0 & 0 & 0 & 0 & 0 & \cdots
\end{array}\right] \text { and } B:=0,
$$

then $\widetilde{A}_{1}$ belongs to $H_{s}^{2}\left(B\left(\mathbb{C}^{2}, \ell^{2}\right)\right)$ such that $\Delta$ and $A_{1}$ are right coprime, $\Delta^{*} B=0$ and $\mathrm{nc}\left\{\Phi_{+}\right\} \leq \operatorname{dim} \ell^{2}=\infty$. Therefore $\Phi=\Delta A_{1}^{*}$ is also a canonical decomposition of $\Phi$. In this case, $\operatorname{ker} H_{\stackrel{\Phi}{*}}^{*} \neq \Delta H_{\ell^{2}}^{2}$. Therefore, the canonical decomposition of $\Phi$ is not unique.

REMARK 5.3. Let $\Delta$ be an inner matrix function with values in $\mathcal{B}\left(E^{\prime}, E\right)$. Then Theorem [5.1] says that if $\operatorname{dim} E^{\prime}<\infty$, the expression (5.5) satisfying the conditions (i) - (iv) in Theorem 5.1 gives ker $H_{\overparen{\Phi}}^{*}=\Delta H_{E^{\prime}}^{2}$. We note that the condition (iv) on nc-number cannot be dropped from the assumptions of Theorem 5.1. To see this, let

If

$$
\Delta:=\frac{1}{\sqrt{2}}\left[\begin{array}{l}
z \\
1
\end{array}\right], \quad A:=\left[\begin{array}{c}
\sqrt{2} \\
0
\end{array}\right] \text { and } \quad B:=0
$$

$$
\Phi:=\Delta A^{*}+B=\left[\begin{array}{ll}
z & 0 \\
1 & 0
\end{array}\right],
$$

then $\Phi$ satisfies the conditions (i), (ii), and (iii), but ker $H_{\overparen{\Phi}}^{*}=z H^{2} \oplus H^{2} \neq \Delta H^{2}$. Note that by Theorem $4.10 \operatorname{nc}\left\{\Phi_{+}\right\}=2$, which does not satisfy the condition on nc-number, say nc $\left\{\Phi_{+}\right\} \leq 1$.

Corollary 5.4. If $\breve{\Delta}$ is of bounded type then $B$ in (5.5) is given by

$$
B=\Delta_{c} \Delta_{c}^{*} \Phi
$$

where $\Delta_{c}$ is the complementary factor of $\Delta$, with values in $\mathcal{B}\left(D^{\prime}, E\right)$. Moreover, if $\operatorname{dim} E^{\prime}<\infty$, then $\operatorname{dim} D^{\prime}$ can be computed by the formula

$$
\operatorname{dim} D^{\prime}=\mathrm{nc}\{\Delta\}-\operatorname{nc}\left\{\Phi_{+}\right\} .
$$

Proof. Suppose that $\breve{\Delta}$ is of bounded type. Then by Corollary 4.20 , $\left[\Delta, \Delta_{c}\right]$ is two-sided inner, where $\Delta_{c}$ is the complementary factor of $\Delta$, with values in $\mathcal{B}\left(D^{\prime}, E\right)$. We thus have

$$
I=\left[\Delta, \Delta_{c}\right]\left[\Delta, \Delta_{c}\right]^{*}=\Delta \Delta^{*}+\Delta_{c} \Delta_{c}^{*},
$$

so that

$$
B=\Phi-\Delta A^{*}=\left(I-\Delta \Delta^{*}\right) \Phi=\Delta_{c} \Delta_{c}^{*} \Phi .
$$


This proves the first assertion. The second assertion follows at once from the facts that $\mathrm{nc}\left\{\Phi_{+}\right\}=\operatorname{dim} E^{\prime}<\infty$ (by Theorem 4.10) and $\mathrm{nc}\{\Delta\}=\operatorname{dim} E^{\prime}+\operatorname{dim} D^{\prime}$ (by Corollary 4.12).

The following corollary is an extension of Lemma 4.2 (the Douglas-ShapiroShields factorization) to strong $L^{2}$-functions.

Corollary 5.5. If $\Phi$ is a strong $L^{2}$-function with values in $\mathcal{B}(D, E)$, then the following are equivalent:

(a) The flip $\breve{\Phi}$ of $\Phi$ is of bounded type;

(b) $\Phi=\Delta A^{*}$ ( $\Delta$ is two-sided inner) is a canonical decomposition of $\Phi$.

Proof. The implication $(\mathrm{a}) \Rightarrow$ (b) follows from the proof of Theorem 5.1, For the implication $(\mathrm{b}) \Rightarrow(\mathrm{a})$, suppose $\Phi=\Delta A^{*}$ ( $\Delta$ is two-sided inner) is a canonical decomposition of $\Phi$. By Lemma 4.4, there exists an inner function $\Theta$ with values in $\mathcal{B}\left(D^{\prime}, E\right)$ such that ker $H_{\overparen{\Phi}}^{*}=\Theta H_{D^{\prime}}^{2}$. Then it follows from Lemma 4.3 that $\Delta H_{E}^{2} \subseteq \operatorname{ker} H_{\overparen{\Phi}}^{*}=\Theta H_{D^{\prime}}^{2}$. Since $\Delta$ is two-sided inner, we have that by Lemma 3.12, $\Theta$ is two-sided inner, and hence the flip $\breve{\Phi}$ of $\Phi$ is of bounded type. This completes the proof.

If $\Delta$ is an inner matrix function such that $\Delta \Delta^{*} \Phi$ is analytic (even though $\breve{\Delta}$ is not of bounded type) then the perturbation part $B$ of the canonical decomposition may be also determined in terms of the complementary factor of $\Delta$.

Corollary 5.6. Let $\Phi$ be an $n \times m$ matrix-valued $H^{2}$-function. Then the following are equivalent:

(a) $\operatorname{ker} H_{\Phi}^{*}=\Delta H_{\mathbb{C}^{r}}^{2}$ for an $n \times r$ inner matrix function $\Delta$ such that $\Delta \Delta^{*} \Phi$ is analytic;

(b) $\Phi=\Delta A^{*}+\Delta_{c} \Delta_{c}^{*} \Phi$ is a canonical decomposition of $\Phi$, where $\Delta_{c}$ is the complementary factor of $\Delta$.

Proof. (a) $\Rightarrow(\mathrm{b})$ : Suppose that ker $H_{\overleftarrow{\Phi}^{*}}^{*}=\Delta H_{\mathbb{C}^{r}}^{2}$ for an $n \times r$ inner matrix function $\Delta$ such that $\Delta \Delta^{*} \Phi$ is analytic. Then by the proof of Theorem 5.1, we can write

$$
\Phi=\Delta A^{*}+B
$$

where $B=\left(I-\Delta \Delta^{*}\right) \Phi$. Write $\Phi \equiv\left[\phi_{1}, \phi_{1}, \cdots, \phi_{m}\right]$. Since $\Delta \Delta^{*} \Phi \in H_{M_{n \times m}}^{2}$ and $\Delta^{*}\left(I-\Delta \Delta^{*}\right)=0$, it follows from Corollary 3.15 and Lemma 4.5 that for each $j=1,2, \cdots m$,

$$
\left(I-\Delta \Delta^{*}\right) \phi_{j} \in \operatorname{ker} \Delta^{*}=\Delta_{c} H_{\mathbb{C}^{p}}^{2}
$$

which implies that $B=\left(I-\Delta \Delta^{*}\right) \Phi=\Delta_{c} D$ for some $D \in H_{M_{p \times m}}^{2}$. Thus

$$
\Delta_{c}^{*} B=\Delta_{c}^{*}\left(I-\Delta \Delta^{*}\right) \Phi=D,
$$

so that

$$
B=\Delta_{c} D=\Delta_{c} \Delta_{c}^{*}\left(I-\Delta \Delta^{*}\right) \Phi=\Delta_{c} \Delta_{c}^{*} \Phi .
$$

(b) $\Rightarrow$ (a): Suppose that $\Phi=\Delta A^{*}+\Delta_{c} \Delta_{c}^{*} \Phi$ is a canonical decomposition of $\Phi$. Since $\Phi$ is a matrix-valued function, it follows from Theorem 5.1 that

$$
\Delta_{c} \Delta_{c}^{*} \Phi=B=\left(I-\Delta \Delta^{*}\right) \Phi,
$$


so that

$$
\Phi=\Delta_{c} \Delta_{c}^{*} \Phi+\Delta \Delta^{*} \Phi
$$

But since $\left\langle\Delta_{c} \Delta_{c}^{*} \phi_{j}, \Delta \Delta^{*} \phi_{j}\right\rangle=0$ for all $j=1,2, \cdots, m$, it follows that $\Delta \Delta^{*} \Phi \in$ $H_{M_{n \times m}}^{2}$. This completes the proof.

Corollary 5.7. Let $\Phi$ be an $n \times m$ matrix-valued $H^{2}$-function satisfying ker $H_{\breve{\Phi}}^{*}=\Delta H_{\mathbb{C}^{r}}^{2}$ for an $n \times r$ inner matrix function $\Delta$ such that $\Delta \Delta^{*}$ is analytic. Then $\Phi$ can be written as

$$
\Phi=\Delta A^{*}+\Delta_{c} C \quad\left(\text { with } C:=P_{+} \Delta_{c}^{*} \Phi \in H_{M_{p \times m}}^{2}\right),
$$

where $\Delta_{c}$ is the complementary factor of $\Delta$.

Proof. We claim that if $\Delta \Delta^{*}$ is analytic, then

$$
\left(I-\Delta \Delta^{*}\right) H_{\mathbb{C}^{n}}^{2}=\Delta_{c} H_{\mathbb{C}^{p}}^{2}
$$

To see this, let $f \in \Delta_{c} H_{\mathbb{C}^{p}}^{2}$. Then $f=\Delta_{c} g$ for some $g \in H_{\mathbb{C}^{p}}^{2}$. Observe that

$$
\left(I-\Delta^{*}\right) f=\left(I-\Delta \Delta^{*}\right) \Delta_{c} g=\Delta_{c} g=f,
$$

which implies that $f \in\left(I-\Delta \Delta^{*}\right) H_{\mathbb{C}^{n}}^{2}$. Thus we have $\Delta_{c} H_{\mathbb{C}^{p}}^{2} \subseteq\left(I-\Delta^{*}\right) H_{\mathbb{C}^{n}}^{2}$. The converse inclusion follows from the proof of Corollary 5.6. This proves (5.15). Thus $I-\Delta \Delta^{*}$ is the orthogonal projection that maps from $H_{\mathbb{C}^{n}}^{2}$ onto $\Delta_{c} H_{\mathbb{C}^{p}}^{2}$. Therefore by the Projection Lemma in [Ni1, P. 43], we have

$$
\left.\left(I-\Delta \Delta^{*}\right)\right|_{H_{\mathbb{C}^{n}}^{2}}=\Delta_{c} P_{+} \Delta_{c}^{*}
$$

so that

as desired

$$
\Phi=\Delta A^{*}+B=\Delta A^{*}+\Delta_{c} P_{+} \Delta_{c}^{*} \Phi,
$$




\section{CHAPTER 6}

\section{The Beurling degree}

We first consider Question 1.5. Question 1.5 can be rephrased as: If $\Delta$ is an inner function with values in $\mathcal{B}\left(E^{\prime}, E\right)$, does there exist a strong $L^{2}$-function $\Phi$ with values in $\mathcal{B}(D, E)$ satisfying the equation

$$
\text { ker } H_{\overleftarrow{\Phi}}^{*}=\Delta H_{E^{\prime}}^{2} ?
$$

To closely understand an answer to Question 1.5. we examine a question whether there exists an inner function $\Omega$ satisfying ker $H_{\Omega^{*}}=\Delta H_{E^{\prime}}^{2}$ if $\Delta$ is an inner function with values in $\mathcal{B}\left(E^{\prime}, E\right)$. In fact, the answer to this question is negative. Indeed, if ker $H_{\Omega^{*}}=\Delta H_{E^{\prime}}^{2}$ for some inner function $\Omega \in H^{\infty}(\mathcal{B}(D, E))$, then by Lemma 4.5. we have $\left[\Omega, \Omega_{c}\right]=\Delta$, and hence $\Delta_{c}=0$. Conversely, if $\Delta_{c}=0$ then by again Lemma 4.5, we should have ker $H_{\Delta^{*}}=\Delta H_{E^{\prime}}^{2}$. Consequently, ker $H_{\Omega^{*}}=\Delta H_{E^{\prime}}^{2}$ for some inner function $\Omega$ if and only if $\Delta_{c}=0$. Thus if

$$
\Delta:=\left[\begin{array}{l}
1 \\
0
\end{array}\right],
$$

then there exists no inner function $\Omega$ such that ker $H_{\Omega^{*}}=\Delta H^{2}$. On the other hand, we note that the solution $\Phi$ is not unique although there exists an inner function $\Phi$ satisfying the equation (6.1). For example, if $\Delta:=\operatorname{diag}(z, 1,1)$, then the following $\Phi$ are such solutions:

$$
\Phi=\left[\begin{array}{l}
z \\
0 \\
0
\end{array}\right], \quad\left[\begin{array}{ll}
z & 0 \\
0 & 1 \\
0 & 0
\end{array}\right], \quad \Delta .
$$

The following theorem gives an affirmative answer to Question 1.5; indeed, we can always find a strong $L^{2}$-function $\Phi$ with values in $\mathcal{B}(D, E)$ satisfying the equation $\operatorname{ker} H_{\Phi}^{*}=\Delta H_{E^{\prime}}^{2}$.

Theorem 6.1. Let $\Delta$ be an inner function with values in $\mathcal{B}\left(E^{\prime}, E\right)$. Then there exists a function $\Phi$ in $H_{s}^{2}(\mathcal{B}(D, E))$, with either $D=E^{\prime}$ or $D=\mathbb{C} \oplus E^{\prime}$, satisfying

$$
\operatorname{ker} H_{\overleftrightarrow{\Phi}}^{*}=\Delta H_{E^{\prime}}^{2} \text {. }
$$

Proof. If ker $\Delta^{*}=\{0\}$, take $\Phi=\Delta$. Then it follows from Lemma 4.5 that

$$
\operatorname{ker} H_{\overleftrightarrow{\Phi}}^{*}=\operatorname{ker} H_{\Delta^{*}}=\Delta H_{E^{\prime}}^{2} .
$$

If instead ker $\Delta^{*} \neq\{0\}$, let $\Delta_{c}$ be the complementary factor of $\Delta$ with values in $\mathcal{B}\left(E^{\prime \prime}, E\right)$ for some nonzero Hilbert space $E^{\prime \prime}$. Choose a cyclic vector $g \in H_{E^{\prime \prime}}^{2}$ of $S_{E^{\prime \prime}}^{*}$ and define

$$
\Phi:=\left[\left[z \Delta_{c} g\right], \Delta\right],
$$


where $\left[z \Delta_{c} g\right](z): \mathbb{C} \rightarrow E$ is given by $\left[z \Delta_{c} g\right](z) \alpha:=\alpha z \Delta_{c}(z) g(z)$. Then it follows from Lemma 3.6 and Corollary 3.15 that $\Phi$ belongs to $H_{s}^{2}(\mathcal{B}(D, E))$, where $D=$ $\mathbb{C} \oplus E^{\prime}$. For each $x \equiv \alpha \oplus x_{0} \in D, f \in H_{E^{\prime}}^{2}$, and $n=1,2,3, \cdots$, we have

$$
\begin{aligned}
\int_{\mathbb{T}}\left\langle\Phi(z) x, z^{n} \Delta(z) f(z)\right\rangle_{E} d m(z) & =\int_{\mathbb{T}}\left\langle\alpha z \Delta_{c}(z) g(z)+\Delta(z) x_{0}, z^{n} \Delta(z) f(z)\right\rangle_{E} d m(z) \\
& =\int_{\mathbb{T}}\left\langle x_{0}, z^{n} f(z)\right\rangle_{E^{\prime}} d m(z) \quad\left(\text { since } \Delta^{*} \Delta_{c}=0\right) \\
& =0 .
\end{aligned}
$$

It thus follows from Lemma 4.3 that

$$
\Delta H_{E^{\prime}}^{2} \subseteq \operatorname{ker} H_{\breve{\Phi}}^{*} .
$$

For the reverse inclusion, suppose $h \in \operatorname{ker} H_{\stackrel{\Phi}{*}}^{*}$. Then by Lemma 4.3, we have that for each $x_{0} \in E^{\prime}$ and $n=1,2,3, \cdots$,

$$
\int_{\mathbb{T}}\left\langle\Delta(z) x_{0}, z^{n} h(z)\right\rangle_{E} d m(z)=0
$$

which implies, by Lemma 4.3, that $h \in$ ker $H_{\Delta^{*}}$. It thus follows from Lemma 4.5 that

$$
\operatorname{ker} H_{\overleftrightarrow{\Phi}}^{*} \subseteq \operatorname{ker} H_{\Delta^{*}}=\Delta H_{E^{\prime}}^{2} \bigoplus \Delta_{c} H_{E^{\prime \prime}}^{2} .
$$

Assume to the contrary that ker $H_{\overparen{\Phi}}^{*} \neq \Delta H_{E^{\prime}}^{2}$. Then by (6.2) and (6.3), there exists a nonzero function $f \in H_{E^{\prime \prime}}^{2}$ such that $\Delta_{c} f \in \operatorname{ker} H_{\Phi}^{*}$. It thus follows from Lemma 4.3 that for each $x \equiv \alpha \oplus x_{0} \in D$ and $n=1,2,3, \cdots$,

$$
\begin{aligned}
0 & =\int_{\mathbb{T}}\left\langle\Phi(z) x, z^{n} \Delta_{c}(z) f(z)\right\rangle_{E} d m(z) \\
& =\int_{\mathbb{T}}\left\langle\alpha z \Delta_{c}(z) g(z)+\Delta(z) x_{0}, z^{n} \Delta_{c}(z) f(z)\right\rangle_{E} d m(z) \\
& =\int_{\mathbb{T}}\left\langle z[g](z) \alpha, z^{n} f(z)\right\rangle_{E^{\prime \prime}} d m(z) \quad\left(\text { since } \Delta^{*} \Delta_{c}=0\right),
\end{aligned}
$$

which implies that $f \in \operatorname{ker} H_{\bar{z}[g]}^{*}$. Since $g$ is a cyclic vector of $S_{E^{\prime \prime}}^{*}$, it thus follows from Lemma 4.7 that

$$
f \in\left(\operatorname{cl} \operatorname{ran} H_{\bar{z}[\breve{g}]}\right)^{\perp}=\left(E_{g}^{*}\right)^{\perp}=\{0\},
$$

which is a contradiction. This completes the proof.

If $\Delta$ is an $n \times r$ inner matrix function, then we can find a solution $\Phi \in H_{M_{n \times m}}^{\infty}$ (with $m \leq r+1$ ) of the equation $\operatorname{ker} H_{\overparen{\Phi}}^{*}=\Delta H_{\mathbb{C}^{r}}^{2}$.

COROLlary 6.2. For a given $n \times r$ inner matrix function $\Delta$, there exists at least a solution $\Phi \in H_{M_{n \times m}}^{\infty}$ (with $m \leq r+1$ ) of the equation ker $H_{\overparen{\Phi}}^{*}=\Delta H_{\mathbb{C}^{r}}^{2}$.

Proof. If ker $\Delta^{*}=\{0\}$, then this is obvious. Let $\operatorname{ker} \Delta^{*} \neq\{0\}$ and $\Delta_{c} \in$ $H_{M_{n \times p}}^{\infty}$ be the complementary factor of $\Delta$. Then by Lemma 4.5 $1 \leq p \leq n-r$. For $j=1,2, \cdots, p$, put

$$
g_{j}:=e^{\frac{1}{z-\alpha_{j}}}
$$


where $\alpha_{j}$ are distinct points in the interval [2,3]. Then it is known that (cf. [Ni1, P. 55])

$$
g:=\left[\begin{array}{c}
g_{1} \\
g_{2} \\
\vdots \\
g_{p}
\end{array}\right] \in H_{\mathbb{C} p}^{\infty}
$$

is a cyclic vector of $S_{\mathbb{C}^{p}}^{*}$. Put $\Phi:=\left[\left[z \Delta_{c} g\right], \Delta\right]$. Then by Lemma 3.6 we have $\Phi \in$ $H_{M_{n \times(r+1)}}^{\infty}$. The same argument as the proof of Theorem 6.1 gives the result.

COROLlary 6.3. If $\Delta$ is an inner function with values in $\mathcal{B}\left(E^{\prime}, E\right)$, then there exists a function $\Phi \in L_{s}^{2}\left(\mathcal{B}(D, E)\right.$ ) (with $D=E^{\prime}$ or $D=\mathbb{C} \oplus E^{\prime}$ ) such that $\Phi \equiv \Delta A^{*}+B$ is the BLH-canonical decomposition of $\Phi$.

Proof. By Theorem 6.1, there exists a function $\Phi \in H_{s}^{2}(\mathcal{B}(D, E))$ such that ker $H_{\Phi}^{*}=\Delta H_{E^{\prime}}^{2}$, with $D=E^{\prime}$ or $D=\mathbb{C} \oplus E^{\prime}$. If we put $A:=\Phi^{*} \Delta$ and $B:=\Phi-\Delta A^{*}$, then by the proof of the first assertion of Theorem 5.1] $\Phi=\Delta A^{*}+B$ is the BLH-canonical decomposition of $\Phi$.

REMARK 6.4. In view of Corollary 6.2, it is reasonable to ask whether such a solution $\Phi \in L_{M_{n \times m}}^{2}$ of the equation ker $H_{\breve{\Phi}}^{*}=\Delta H_{\mathbb{C}^{r}}^{2}(\Delta$ an $n \times r$ inner matrix function) exists for each $m=1,2, \cdots$ even though it exists for some $m$. For example, let

$$
\Delta:=\frac{1}{\sqrt{2}}\left[\begin{array}{l}
z \\
1
\end{array}\right]
$$

Then, by Corollary 6.2 there exists a solution $\Phi \in L_{M_{2 \times m}}^{2}(m=1$ or 2$)$ of the equation $\operatorname{ker} H_{\overparen{\Phi}}^{*}=\Delta H^{2}$. For $m=2$, let

$$
\Phi:=\left[\begin{array}{cc}
z & z a \\
1 & -a
\end{array}\right] \in H_{M_{2}}^{\infty}
$$

where $a \in H^{\infty}$ is such that $\bar{a}$ is not of bounded type. Then a direct calculation shows that $\operatorname{ker} H_{\overparen{\Phi}}^{*}=\operatorname{ker} H_{\Phi^{*}}=\Delta H^{2}$. We may then ask how about the case $m=1$. In this case, the answer is affirmative. To see this, let

$$
\Psi:=\left[\begin{array}{c}
z+z a \\
1-a
\end{array}\right] \in H_{M_{2 \times 1}}^{\infty}
$$

where $a \in H^{\infty}$ is such that $\bar{a}$ is not of bounded type. Then a direct calculation shows that ker $H_{\Psi^{*}}=\Delta H^{2}$. Therefore, if $\Delta$ is given by (6.4), then we may assert that there exists a solution $\Phi \in L_{M_{n \times m}}^{2}$ of the equation ker $H_{\overparen{\Phi}}^{*}=\Delta H^{2}$ for each $m=1,2$. However, this assertion is not true in general, i.e., a solution exists for some $m$, but may not exist for another $m_{0}<m$. To see this, let

$$
\Delta:=\left[\begin{array}{ccc}
z & 0 & 0 \\
0 & z & 0 \\
0 & 0 & 1 \\
0 & 0 & 0
\end{array}\right] \in H_{M_{4 \times 3}}^{\infty} .
$$

Then $\Delta$ is inner. We will show that there exists no solution $\Phi \in L_{M_{4 \times 1}}^{2}$ (i.e., the case $m=1$ ) of the equation ker $H_{\overparen{\Phi}}^{*}=\Delta H_{\mathbb{C}^{3}}^{2}$. Assume to the contrary that $\Phi \in L_{M_{4 \times 1}}^{2}$ 
is a solution of the equation $\operatorname{ker} H_{\stackrel{\Phi}{*}}^{*}=\Delta H_{\mathbb{C}^{3}}^{2}$. By Theorem [5.1] $\Phi$ can be written as

$$
\Phi=\Delta A^{*}+B
$$

where $A \in H_{M_{1 \times 3}}^{2}$ is such that $\Delta$ and $A$ are right coprime. But since $\widetilde{\Delta} H_{\mathbb{C}^{4}}^{2}=$ $z H^{2} \oplus z H^{2} \oplus H^{2}$, it follows that

$$
\widetilde{\Delta} H_{\mathbb{C}^{4}}^{2} \bigvee \widetilde{A} H^{2} \neq H_{\mathbb{C}^{3}}^{2}
$$

which implies that $\Delta$ and $A$ are not right coprime, a contradiction. Therefore we cannot find any solution $\Phi$, in $L_{M_{4 \times 1}}^{2}$ (the case $m=1$ ), of the equation ker $H_{\vec{\Phi}}^{*}=$ $\Delta H_{\mathbb{C}^{3}}^{2}$. By contrast, if $m=2$, then we can find a solution $\Phi \in L_{M_{4 \times 2}}^{2}$. Indeed, let

$$
\Phi:=\left[\begin{array}{cc}
z & 0 \\
0 & z \\
0 & 0 \\
a & 0
\end{array}\right],
$$

where $a \in H^{\infty}$ is such that $\bar{a}$ is not of bounded type. Then $\operatorname{ker} H_{\Phi^{*}}=z H^{2} \oplus$ $z H^{2} \oplus H^{2} \oplus\{0\}=\Delta H_{\mathbb{C}^{3}}^{2}$. Thus we obtain a solution for $m=2$ although there exists no solution for $m=1$.

Let $\Delta$ be an inner function with values in $\mathcal{B}\left(E^{\prime}, E\right)$. In view of Remark 6.4 we may ask how to determine a possible dimension of $D$ for which there exists a solution $\Phi \in L_{s}^{2}(\mathcal{B}(D, E))$ of the equation $\operatorname{ker} H_{\stackrel{\Phi}{*}}^{*}=\Delta H_{E^{\prime}}^{2}$. In fact, if we have a solution $\Phi \in L_{s}^{2}(\mathcal{B}(D, E))$ of the equation $\operatorname{ker} H_{\stackrel{\Phi}{*}}^{*}=\Delta H_{E^{\prime}}^{2}$, then a solution $\left.\Psi \in L_{s}^{2}\left(D^{\prime}, E\right)\right)$ also exists if $D^{\prime}$ is a separable complex Hilbert space containing $D$ : indeed, if $\mathbf{0}$ denotes the zero operator in $\mathcal{B}\left(D^{\prime} \ominus D, E\right)$ and $\Psi:=[\Phi, \mathbf{0}]$, then it follows from Lemma 4.3 that $\operatorname{ker} H_{\widetilde{\Phi}}^{*}=\operatorname{ker} H_{\Psi}^{*}$. Thus we would like to ask what is the infimum of $\operatorname{dim} D$ such that there exists a solution $\Phi \in L_{s}^{2}(\mathcal{B}(D, E))$ of the equation ker $H_{\overparen{\Phi}}^{*}=\Delta H_{E^{\prime}}^{2}$. To answer this question, we introduce a notion of the "Beurling degree" for an inner function, by employing a canonical decomposition of strong $L^{2}$-functions induced by the given inner function.

Definition 6.5. Let $\Delta$ be an inner function with values in $\mathcal{B}\left(E^{\prime}, E\right)$. Then the Beurling degree of $\Delta$, denoted by $\operatorname{deg}_{B}(\Delta)$, is defined by

$$
\begin{aligned}
\operatorname{deg}_{B}(\Delta):=\inf \left\{\operatorname{dim} D \in \mathbb{Z}_{+} \cup\{\infty\}: \exists \text { a pair }(A, B)\right. \text { such that } \\
\left.\Phi=\Delta A^{*}+B \text { is a canonical decomposition of } \Phi \in L_{s}^{2}(B(D, E))\right\}
\end{aligned}
$$

Note. By Corollary 6.3 $\operatorname{deg}_{B}(\Delta)$ is well-defined: indeed, $1 \leq \operatorname{deg}_{B}(\Delta) \leq 1+\operatorname{dim} E^{\prime}$. In particular, if $E^{\prime}=\{0\}$, then $\operatorname{deg}_{B}(\Delta)=1$. Also if $\Delta$ is a unitary operator then clearly, $\operatorname{deg}_{B}(\Delta)=1$.

We are ready for:

Theorem 6.6. (The Beurling degree and the spectral multiplicity) Given an inner function $\Delta$ with values in $\mathcal{B}\left(E^{\prime}, E\right)$, with $\operatorname{dim} E^{\prime}<\infty$, let $T:=\left.S_{E}^{*}\right|_{\mathcal{H}(\Delta)}$. Then

$$
\mu_{T}=\operatorname{deg}_{B}(\Delta)
$$


Proof. Let $T:=\left.S_{E}^{*}\right|_{\mathcal{H}(\Delta)}$. We first claim that

$$
\begin{array}{r}
\operatorname{deg}_{B}(\Delta)=\inf \left\{\operatorname{dim} D: \operatorname{ker} H_{\Phi}^{*}=\Delta H_{E^{\prime}}^{2} \text { for some } \Phi \in L_{s}^{2}(\mathcal{B}(D, E))\right. \\
\text { with } D \neq\{0\}\} .
\end{array}
$$

To see this, let $\Delta$ be an inner function with values in $\mathcal{B}\left(E^{\prime}, E\right)$, with $\operatorname{dim} E^{\prime}<\infty$. Suppose that $\Phi=\Delta A^{*}+B$ is a canonical decomposition of $\Phi$ in $L_{s}^{2}(\mathcal{B}(D, E))$. Then by the uniqueness of $\Delta$ in Theorem 5.1 we have

$$
\operatorname{ker} H_{\breve{\Phi}}^{*}=\Delta H_{E^{\prime}}^{2},
$$

which implies

$$
\begin{array}{r}
\operatorname{deg}_{B}(\Delta) \geq \inf \left\{\operatorname{dim} D: \operatorname{ker} H_{\Phi}^{*}=\Delta H_{E^{\prime}}^{2} \text { for some } \Phi \in L_{s}^{2}(\mathcal{B}(D, E))\right. \\
\text { with } D \neq\{0\}\} .
\end{array}
$$

For the reverse inequality of (6.9), suppose $\Phi \in L_{s}^{2}(\mathcal{B}(D, E))$ satisfies ker $H_{\Phi}^{*}=$ $\Delta H_{E^{\prime}}^{2}$. Then by the same argument as in the proof of the first assertion of Theorem 5.1 .

$$
\Phi=\Delta A^{*}+B \quad\left(A:=\Phi^{*} \Delta \text { and } B:=\Phi-\Delta A^{*}\right)
$$

is a canonical decomposition of $\Phi$, and hence we have the reverse inequality of (6.9). This proves the claim (6.7). We will next show that

$$
\operatorname{deg}_{B}(\Delta) \leq \mu_{T} .
$$

If $\mu_{T}=\infty$, then (6.10) is trivial. Suppose $p \equiv \mu_{T}<\infty$. Then there exists a subset $G=\left\{g_{1}, g_{2}, \cdots g_{p}\right\} \subseteq H_{E}^{2}$ such that $E_{G}^{*}=\mathcal{H}(\Delta)$. Put

$$
\Psi:=z[G] .
$$

Then by Lemma 3.6, $\Psi \in H_{s}^{2}\left(\mathcal{B}\left(\mathbb{C}^{p}, E\right)\right)$. It thus follows from Lemma 4.7 that

$$
\mathcal{H}(\Delta)=E_{G}^{*}=\operatorname{cl} \operatorname{ran} H_{\bar{z}[\breve{G}]}=\operatorname{cl} \operatorname{ran} H_{\breve{\Psi}},
$$

which implies ker $H_{\Psi}^{*}=\Delta H_{E^{\prime}}^{2}$. Thus by [6.7], $\operatorname{deg}_{B}(\Delta) \leq p=\mu_{T}$, which proves (6.10). For the reverse inequality of (6.10), suppose that $r \equiv \operatorname{dim} E^{\prime}<\infty$, Write $m_{0} \equiv \operatorname{deg}_{B}(\Delta)$. Then it follows from Theorem 6.1 and (6.7) that $m_{0} \leq r+1<\infty$ and there exists a function $\Phi \in L_{s}^{2}\left(\mathcal{B}\left(\mathbb{C}^{m_{0}}, E\right)\right)$ such that

$$
\operatorname{ker} H_{\overparen{\Phi}}^{*}=\Delta H_{\mathbb{C}^{r}}^{2} \text {. }
$$

Now let

$$
G:=\Phi_{+}-\widehat{\Phi}(0) .
$$

Thus we may write $G=z F$ for some $F \in H_{s}^{2}\left(\mathcal{B}\left(\mathbb{C}^{m_{0}}, E\right)\right)$. Then by Lemma 4.1 and Lemma 4.7, we have that

$$
E_{\{F\}}^{*}=\operatorname{cl} \operatorname{ran} H_{\breve{G}}=\left(\operatorname{ker} H_{\breve{\Phi}}^{*}\right)^{\perp}=\mathcal{H}(\Delta),
$$

which implies $\mu_{T} \leq m_{0}=\operatorname{deg}_{B}(\Delta)$. This completes the proof.

Corollary 6.7. Let $T:=\left.S_{E}^{*}\right|_{\mathcal{H}(\Delta)}$. If $\operatorname{rank}\left(I-T^{*} T\right)<\infty$, then

$$
\mu_{T}=\operatorname{deg}_{B}(\Delta) .
$$


Proof. This follows at once from Theorem 6.6 together with the observation that if $\Delta$ is an inner function with values in $\mathcal{B}\left(E^{\prime}, E\right)$, then $\operatorname{dim} E^{\prime} \leq \operatorname{dim} E=$ $\operatorname{rank}\left(I-T^{*} T\right)<\infty$, where the second equality comes from the Model Theorem (cf. p[6] paragraph containing (1.4) ).

REMARK 6.8. We conclude with some observations on Theorem 6.6

(a) From a careful analysis of the proof of Theorem 6.6 we can see that 6.10) holds in general without the assumption " $\operatorname{dim} E^{\prime}<\infty$ ": more concretely, given an inner function $\Delta$ with values in $\mathcal{B}\left(E^{\prime}, E\right)$, if $T:=\left.S_{E}^{*}\right|_{\mathcal{H}(\Delta)}$, then

$$
\operatorname{deg}_{B}(\Delta) \leq \mu_{T} .
$$

(b) From Remark 6.4 and (6.7), we see that if

$$
\Delta:=\left[\begin{array}{ccc}
z & 0 & 0 \\
0 & z & 0 \\
0 & 0 & 1 \\
0 & 0 & 0
\end{array}\right],
$$

then $\operatorname{deg}_{B}(\Delta)=2$. Let $T:=\left.S_{\mathbb{C}^{4}}^{*}\right|_{\mathcal{H}(\Delta)}$. Observe that

$$
\mathcal{H}(\Delta)=\mathcal{H}(z) \oplus \mathcal{H}(z) \oplus\{0\} \oplus H^{2} .
$$

Since $\mathcal{H}(z) \oplus \mathcal{H}(z)$ has no cyclic vector, we must have $\mu_{T} \neq 1$. In fact, if we put

$$
f=\left[\begin{array}{l}
1 \\
0 \\
0 \\
a
\end{array}\right] \text { and } g=\left[\begin{array}{l}
0 \\
1 \\
0 \\
0
\end{array}\right],
$$

where $\bar{a}$ is not of bounded type, then $E_{\{f, g\}}^{*}=\mathcal{H}(\Delta)$, which implies $\mu_{T}=$ 2. This illustrates Theorem 6.6.

We now answer Question 1.1(ii) in the affirmative.

Remark 6.9. Suppose $\Delta$ is an inner function with values in $\mathcal{B}\left(E^{\prime}, E\right)$, with $\operatorname{dim} E^{\prime}<\infty$. If $\Phi=\Delta A^{*}+B$ is a canonical decomposition of $\Phi$ in $L_{s}^{2}(\mathcal{B}(D, E))$. Then by Theorem 5.1, we have

$$
\operatorname{ker} H_{\overleftrightarrow{\Phi}}^{*}=\Delta H_{E^{\prime}}^{2}
$$

It thus follows from the proof of Theorem 6.6 that

$$
E_{\{F\}}^{*}=\mathcal{H}(\Delta)
$$

where $F$ is defined by

$$
F(z):=\bar{z}\left(\Phi_{+}(z)-\hat{\Phi}(0)\right) .
$$

This gives an answer to the problem of describing the set $\{F\}$ in $H_{E}^{2}$ such that $\mathcal{H}(\Delta)=E_{\{F\}}^{*}$, given an inner function $\Delta$ with values in $\mathcal{B}\left(E^{\prime}, E\right)$, with $\operatorname{dim} E^{\prime}<\infty$. 


\section{CHAPTER 7}

\section{The spectral multiplicity of model operators}

In this chapter, we consider Question 1.6] Let $T:=\left.S_{E}^{*}\right|_{\mathcal{H}(\Delta)}$. For which inner function $\Delta$ with values in $\mathcal{B}\left(E^{\prime}, E\right)$, does it follow that

$$
T \text { is multiplicity-free, i.e., } \mu_{T}=1 \text { ? }
$$

If $\operatorname{dim} E^{\prime}<\infty$, then in the viewpoint of Theorem 6.6. Question 1.6 is equivalent to the following: if $T$ is the truncated backward shift $\left.S_{E}^{*}\right|_{\mathcal{H}(\Delta)}$, which inner function $\Delta$ guarantees that $\operatorname{deg}_{B}(\Delta)=1$ ? To answer Question [1.6 in Section 7.1, we consider the notion of the characteristic scalar inner function of operator-valued inner functions having a meromorphic pseudo-continuation of bounded type in $\mathbb{D}^{e} \equiv$ $\{z: 1<|z| \leq \infty\}$. In Section 7.2, we give an answer to Question 1.6 In Section 7.3 , we consider a reduction to the case of $C_{0}$-contractions for the spectral multiplicity of model operators.

\section{$\S$ 7.1. Characteristic scalar inner functions}

In this section we consider the characteristic scalar inner functions of operatorvalued inner functions, by using the results of Section 4.5. The characteristic scalar inner function of a two-sided inner matrix function has been studied in [Hel], SFBK and CHL3.

Let $\Delta \in H^{\infty}(\mathcal{B}(D, E))$ have a meromorphic pseudo-continuation of bounded type in $\mathbb{D}^{e}$. Then by Lemma 4.23 there exists a scalar inner function $\delta$ such that $\delta H_{E}^{2} \subseteq \operatorname{ker} H_{\Delta^{*}} . \quad$ Put $G:=\delta \Delta^{*} \in H^{\infty}(\mathcal{B}(E, D))$. If further $\Delta$ is inner then $G \Delta=\delta I_{D}$, so that

$$
\text { g.c.d. }\left\{\delta: G \Delta=\delta I_{D} \text { for some } G \in H^{\infty}(\mathcal{B}(E, D))\right\}
$$

always exists. Thus the following definition makes sense.

Definition 7.1. Let $\Delta$ be an inner function with values in $\mathcal{B}(D, E)$. If $\Delta$ has a meromorphic pseudo-continuation of bounded type in $\mathbb{D}^{e}$, define

$$
m_{\Delta}:=\text { g.c.d. }\left\{\delta: G \Delta=\delta I_{D} \text { for some } G \in H^{\infty}(\mathcal{B}(E, D))\right\},
$$

where $\delta$ is a scalar inner function. The inner function $m_{\Delta}$ is called the characteristic scalar inner function of $\Delta$.

We note that if $\left.T \equiv P_{\mathcal{H}(\Delta)} S_{E}\right|_{\mathcal{H}(\Delta)} \in C_{0}$, then $m_{\Delta}$ coincides with the minimal annihilator $m_{T}$ of $T$ (cf. Ber, [SFBK], CHL3). 
We would like to remark that

$$
\text { g.c.d. }\left\{\delta: G \Delta=\delta I_{D} \text { for some } G \in H^{\infty}(\mathcal{B}(E, D))\right\}
$$

may exist for some inner function $\Delta$ having no meromorphic pseudo-continuation of bounded type in $\mathbb{D}^{e}$. To see this, let

$$
\Delta:=\left[\begin{array}{l}
f \\
g
\end{array}\right] \quad\left(f, g \in H^{\infty}\right),
$$

where $f$ and $g$ are given in Example 3.11. Then $\Delta$ is an inner function. Since $\breve{f}$ is not of bounded type it follows from Corollary 4.27 that $\Delta$ has no meromorphic pseudo-continuation of bounded type in $\mathbb{D}^{e}$. On the other hand, since $\Delta$ is inner, by the Complementing Lemma, there exists a function $G \in H_{M_{1 \times 2}}^{\infty}$ such that $G \Delta$ is a scalar inner function, so that (7.1) exists.

If $\Delta$ is an $n \times n$ square inner matrix function then we may write $\Delta \equiv\left[\theta_{i j} \bar{b}_{i j}\right]$, where $\theta_{i j}$ is inner and $\theta_{i j}$ and $b_{i j} \in H^{\infty}$ are coprime for each $i, j=1,2, \cdots, n$. In Lemma 4.12 of CHL3, it was shown that

$$
m_{\Delta}=\text { l.c.m. }\left\{\theta_{i j}: i, j=1,2, \cdots, n\right\} .
$$

In this section, we examine the cases of general inner functions that have meromorphic pseudo-continuations of bounded type in $\mathbb{D}^{e}$.

On the other hand, if $\Phi \in H^{\infty}(\mathcal{B}(D, E))$ has a meromorphic pseudo-continuation of bounded type in $\mathbb{D}^{e}$, then by Lemma 4.23, $\delta H_{E}^{2} \subseteq$ ker $H_{\Phi^{*}}$ for some scalar inner function $\delta$. Thus we may also define

$$
\omega_{\Phi}:=\text { g.c.d. }\left\{\delta: \delta H_{E}^{2} \subseteq \text { ker } H_{\Phi^{*}} \text { for some scalar inner function } \delta\right\} .
$$

If $\Delta$ is an inner function with values in $\mathcal{B}(D, E)$ and has a meromorphic pseudocontinuation of bounded type in $\mathbb{D}^{e}$, then $\omega_{\Delta}$ is called the pseudo-characteristic scalar inner function of $\Delta$. Note that $m_{\Delta}$ is an inner divisor of $\omega_{\Delta}$. If further $\Delta$ is two-sided inner, then

$$
\delta H_{E}^{2} \subseteq \operatorname{ker} H_{\Delta^{*}} \Longleftrightarrow G \equiv \delta \Delta^{*} \in H^{\infty}(\mathcal{B}(E)) \Longleftrightarrow G \Delta=\Delta G=\delta I_{E},
$$

which implies $m_{\Delta}=\omega_{\Delta}$.

The following lemma shows a way to determine $\omega_{\Phi}$ more easily.

Lemma 7.2. Let $D$ and $E$ be separable complex Hilbert spaces and let $\left\{d_{j}\right\}$ and $\left\{e_{i}\right\}$ be orthonormal bases of $D$ and $E$, respectively. Suppose $\Phi \in H^{\infty}(\mathcal{B}(D, E))$ has a meromorphic pseudo-continuation of bounded type in $\mathbb{D}^{e}$. In view of Proposition 4.25, we may write

$$
\phi_{i j} \equiv\left\langle\Phi d_{j}, e_{i}\right\rangle_{E}=\theta_{i j} \bar{a}_{i j}
$$

where $\theta_{i j}$ is inner and $\theta_{i j}$ and $a_{i j} \in H^{\infty}$ are coprime. Then we have

$$
\omega_{\Phi}=\text { l.c.m. }\left\{\theta_{i j}: i, j=1,2, \cdots,\right\} \text {. }
$$

Proof. Let $\Phi \in H^{\infty}(\mathcal{B}(D, E))$ have a meromorphic pseudo-continuation of bounded type in $\mathbb{D}^{e}$. By Lemma 4.23, we may write $\Phi=\theta A^{*}$ for some $A \in$ $H^{\infty}(\mathcal{B}(E, D))$ and a scalar inner function $\theta$. Also by an analysis of the proof of Proposition 4.25. we can see that $\theta_{0} \equiv$ l.c.m. $\left\{\theta_{i j}: i, j=1,2, \cdots,\right\}$ is an inner divisor of $\theta$. Thus by Lemma 4.23, $\theta_{0}$ is an inner divisor of $\omega_{\Phi}$. Since $\Phi \in H^{\infty}(\mathcal{B}(D, E))$, it follows that for all $f \in H_{E}^{2}$ and $j, n \geq 1$,

$$
\left\langle\Phi(z) d_{j}, z^{n} \theta_{0}(z) f(z)\right\rangle_{E} \in L^{2}
$$


On the other hand, for all $f \in H_{E}^{2}$,

$$
f(z)=\sum_{i \geq 1}\left\langle f(z), e_{i}\right\rangle e_{i} \equiv \sum_{i \geq 1} f_{i}(z) e_{i} \quad \text { for almost all } z \in \mathbb{T} \quad\left(f_{i} \in H^{2}\right) .
$$

Since $\theta_{0}=$ l.c.m. $\left\{\theta_{i j}: i, j=1,2, \cdots,\right\}$, it follows from (7.4) and (7.5) that for all $j, n \geq 1$,

$$
\begin{aligned}
\int_{\mathbb{T}}\left\langle\Phi(z) d_{j}, z^{n} \theta_{0}(z) f(z)\right\rangle_{E} d m(z) & =\int_{\mathbb{T}} \bar{z}^{n} \sum_{i \geq 1} \overline{f_{i}}(z) \overline{\theta_{0}}(z) \theta_{i j}(z) \bar{a}_{i j}(z) d m(z) \\
& =0,
\end{aligned}
$$

where the last equality follows from the fact that $\bar{z}^{n} \sum_{i \geq 1} \overline{f_{i}}(z) \overline{\theta_{0}}(z) \theta_{i j}(z) \bar{a}_{i j}(z) \in$ $L^{2} \ominus H^{2}$. Since $\left\{d_{i}\right\}$ is an orthonormal basis for $D$, it follows from Fatou's Lemma that for all $x \in D$ and $n=1,2,3, \cdots$,

$$
\int_{\mathbb{T}}\left\langle\Phi(z) x, z^{n} \theta_{0}(z) f(z)\right\rangle_{E} d m(z)=0 .
$$

Thus by Lemma 4.3, $\theta_{0} H_{E}^{2} \subseteq$ ker $H_{\Phi^{*}}$, so that $\omega_{\Phi}$ is an inner divisor of $\theta_{0}$, and therefore $\theta_{0}=\omega_{\Phi}$. This complete the proof.

Corollary 7.3. Let $\Delta$ be a two-sided inner matrix function. Thus, in view of Corollary 4.27, we may write $\Delta \equiv\left[\theta_{i j} \bar{b}_{i j}\right]$, where $\theta_{i j}$ is an inner function and $\theta_{i j}$ and $b_{i j} \in H^{\infty}$ are coprime for each $i, j=1,2, \cdots$. Then

$$
\omega_{\Delta}=m_{\Delta}=\text { l.c.m. }\left\{\theta_{i j}: i, j=1,2, \cdots,\right\} .
$$

Proof. Immediate from Lemma 7.2

REMARK 7.4. If $\Delta$ is not two-sided inner then Corollary 7.3 may fail. To see this, let

$$
\Delta:=\frac{1}{\sqrt{2}}\left[\begin{array}{l}
1 \\
z
\end{array}\right] .
$$

Then by Corollary 4.27, $\Delta$ has a meromorphic pseudo-continuation of bounded type in $\mathbb{D}^{e}$. It thus follows from Lemma 7.2 that $\omega_{\Delta}=z$. On the other hand, let $G:=\left[\begin{array}{ll}\sqrt{2} & 0\end{array}\right]$. Then $G \Delta=1$, so that $m_{\Delta}=1 \neq z=\omega_{\Delta}$. Note that, by Corollary 7.3 ,

$$
\left[\Delta, \Delta_{c}\right]=\frac{1}{\sqrt{2}}\left[\begin{array}{cc}
1 & 1 \\
z & -z
\end{array}\right] \text { and } m_{\left[\Delta, \Delta_{c}\right]}=\omega_{\left[\Delta, \Delta_{c}\right]}=z .
$$

The following lemma shows that Remark 7.4 is not an accident.

Lemma 7.5. Let $\Delta$ be an inner function and have a meromorphic pseudocontinuation of bounded type in $\mathbb{D}^{e}$. Then

$$
m_{\left[\Delta, \Delta_{c}\right]}=\omega_{\left[\Delta, \Delta_{c}\right]}=\omega_{\Delta}
$$

and $\Delta_{c}$ has a meromorphic pseudo-continuation of bounded type in $\mathbb{D}^{e}$ : in this case, $\omega_{\Delta_{c}}$ is an inner divisor of $\omega_{\Delta}$. 
Proof. Suppose that $\Delta$ is an inner function with values in $\mathcal{B}(D, E)$ and has a meromorphic pseudo-continuation of bounded type in $\mathbb{D}^{e}$. Then it follows from Corollary 4.20 and Lemma 4.22 that $\left[\Delta, \Delta_{c}\right]$ is two-sided inner. On the other hand, it follows from Lemma 4.5 that

$$
\operatorname{ker} H_{\Delta^{*}}=\left[\Delta, \Delta_{c}\right] H_{D \oplus D^{\prime}}^{2}=\operatorname{ker} H_{\left[\Delta, \Delta_{c}\right]^{*}} .
$$

Thus by Lemma 4.23. $\left[\Delta, \Delta_{c}\right]$ has a meromorphic pseudo-continuation of bounded type in $\mathbb{D}^{e}$ and $m_{\left[\Delta, \Delta_{c}\right]}=\omega_{\left[\Delta, \Delta_{c}\right]}=\omega_{\Delta}$. This proves the first assertion. Since $\left[\Delta, \Delta_{c}\right]$ has a meromorphic pseudo-continuation of bounded type in $\mathbb{D}^{e}$, it follows from Lemma 4.23 that $\Delta_{c}$ has a meromorphic pseudo-continuation of bounded type in $\mathbb{D}^{e}$. On the other hand, by Lemma 4.5(b), $\Delta_{c}^{*} \Delta=0$. Thus, by Lemma 4.5(a), $\Delta H_{D}^{2} \subseteq \operatorname{ker} \Delta_{c}^{*}=\Delta_{c c} H_{D^{\prime \prime}}^{2}$, which implies that $\Delta_{c c}$ is a left inner divisor of $\Delta$. Thus, $\left[\Delta_{c c}, \Delta_{c}\right]$ is a left inner divisor of $\left[\Delta, \Delta_{c}\right]$, so that $\omega_{\Delta_{c}}=\omega_{\left[\Delta_{c c}, \Delta_{c}\right]}$ is an inner divisor of $\omega_{\Delta}=\omega_{\left[\Delta, \Delta_{c}\right]}$. This proves the second assertion.

\section{$\S$ 7.2. Multiplicity-free model operators}

In this section we give an answer to Question 1.6. This is accomplished by several lemmas.

LEMma 7.6. Let $\Phi \in H^{\infty}(\mathcal{B}(D, E))$ have a meromorphic pseudo-continuation of bounded type in $\mathbb{D}^{e}$. Then for each cyclic vector $g$ of $S_{D}^{*}$,

$$
\operatorname{ker} H_{[z \Phi g]^{\smile}}^{*}=\operatorname{ker} \Phi^{*},
$$

where $[z \Phi g]^{\smile}$ denotes the flip of $[z \Phi g]$.

Proof. Let $\Phi \in H^{\infty}(\mathcal{B}(D, E))$ have a meromorphic pseudo-continuation of bounded type in $\mathbb{D}^{e}$. Then by Lemma 4.23, there exists a scalar inner function $\delta$ such that $\delta H_{E}^{2} \subseteq \operatorname{ker} H_{\Phi^{*}}$. We thus have

$$
\delta \Phi^{*} h \in H_{D}^{2} \quad \text { for any } h \in H_{E}^{2} .
$$

Let $g$ be a cyclic vector of $S_{D}^{*}$ and $h \in \operatorname{ker} H_{[z \Phi g]}^{*}$. Then it follows from Lemma 4.3 that for all $n=1,2,3 \cdots$,

$$
\begin{aligned}
0 & =\int_{\mathbb{T}}\left\langle z \Phi(z) g(z), z^{n} \delta(z) h(z)\right\rangle_{E} d m(z) \\
& =\int_{\mathbb{T}}\left\langle S_{D}^{*(n-1)} g(z), \delta(z) \Phi^{*}(z) h(z)\right\rangle_{D} d m(z) \\
& =\left\langle S_{D}^{*(n-1)} g(z), \delta(z) \Phi^{*}(z) h(z)\right\rangle_{L_{D}^{2}},
\end{aligned}
$$

which implies, by (7.6), that $\delta \Phi^{*} h=0$, and hence $h \in \operatorname{ker} \Phi^{*}$. We thus have

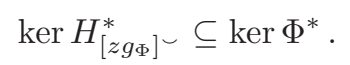

The reverse inclusion follows at once from Lemma 4.3. This completes the proof. 
Lemma 7.7. Let $\Phi \in H^{\infty}(\mathcal{B}(D, E))$ have a meromorphic pseudo-continuation of bounded type in $\mathbb{D}^{e}$. Then for each cyclic vector $g$ of $S_{D}^{*}$,

$$
E_{\{\Phi g\}}^{*}=\mathcal{H}\left(\left(\Phi^{i}\right)_{c}\right)
$$

where $\Phi^{i}$ denotes the inner part in the inner-outer factorization of $\Phi$. Hence, in particular, $\left.S_{E}^{*}\right|_{\mathcal{H}\left(\left(\Phi^{i}\right)_{c}\right)}$ is multiplicity-free.

Proof. Let $\Phi \equiv \Phi^{i} \Phi^{e}$ be the inner-outer factorization of $\Phi$. Since $\Phi^{e}$ has dense range, $\left(\Phi^{e}\right)^{*}$ is one-one, so that $\operatorname{ker} \Phi^{*}=\operatorname{ker}\left(\Phi^{i}\right)^{*}$. It thus follows from Lemma 4.5. Lemma 4.7 and Lemma 7.6 that

$$
E_{\{\Phi g\}}^{*}=\left(\operatorname{ker} H_{[z \Phi g]^{\smile}}^{*}\right)^{\perp}=\left(\operatorname{ker} \Phi^{*}\right)^{\perp}=\mathcal{H}\left(\left(\Phi^{i}\right)_{c}\right),
$$

which proves (7.7). This completes the proof.

The following corollary is a matrix-valued version of Lemma 7.7 .

Corollary 7.8. Let $\Delta$ be an $n \times r$ inner matrix function such that $\breve{\Delta}$ is of bounded type. If $g$ is a cyclic vector of $S_{\mathbb{C}^{r}}^{*}$, then $E_{\{\Delta g\}}^{*}=\mathcal{H}\left(\Delta_{c}\right)$.

PROOF. It follows from Corollary 4.27 and Lemma 7.7

The following lemma shows that the flip of the adjoint of an inner function may be an outer function.

Lemma 7.9. Let $\Delta$ be an inner function with values in $\mathcal{B}(D, E)$, with its complementary factor $\Delta_{c}$ with values in $\mathcal{B}\left(D^{\prime}, E\right)$. If $\operatorname{dim} D^{\prime}<\infty$, then $\widetilde{\Delta_{c}}$ is an outer function. Write

Proof. If $D^{\prime}=\{0\}$, then this is trivial. Suppose that $D^{\prime}=\mathbb{C}^{p}$ for some $p \geq 1$.

$$
\widetilde{\Delta_{c}} \equiv\left(\widetilde{\Delta_{c}}\right)^{i}\left(\widetilde{\Delta_{c}}\right)^{e} \quad \text { (inner-outer factorization), }
$$

where $\left(\widetilde{\Delta_{c}}\right)^{i} \in H_{M_{p \times q}}^{\infty}$ and $\left(\widetilde{\Delta_{c}}\right)^{e} \in H^{\infty}\left(\mathcal{B}\left(E, \mathbb{C}^{q}\right)\right)$ for some $q \leq p$. It thus follows that

$$
q=\operatorname{Rank}\left(\widetilde{\Delta_{c}}\right)^{i} \geq \operatorname{Rank} \widetilde{\Delta_{c}}=\max _{\zeta \in \mathbb{D}} \operatorname{rank} \widetilde{\Delta_{c}}(\zeta) \widetilde{\Delta_{c}}(\zeta)^{*}=p,
$$

which implies $p=q$. Since $\left(\widetilde{\Delta_{c}}\right)^{i} \in H_{M_{p}}^{\infty}$ is two-sided inner, by the Complementing Lemma, there exists a function $G \in H_{M_{p}}^{\infty}$ and a scalar inner function $\theta$ such that $G\left(\widetilde{\Delta_{c}}\right)^{i}=\theta I_{p}$. Thus by (77.8), we have $G \widetilde{\Delta_{c}}=\theta I_{p}\left(\widetilde{\Delta_{c}}\right)^{e}$, and hence we have

$$
\breve{\theta} I_{E} \Delta_{c} \widetilde{G}=\widetilde{\theta} \widetilde{I_{p} G \widetilde{\Delta_{c}}}=\widetilde{\left(\widetilde{\Delta_{c}}\right)^{e}} \in H^{\infty}\left(\mathcal{B}\left(\mathbb{C}^{p}, E\right)\right) .
$$

Thus we have

$$
\breve{\theta} I_{E} \Delta_{c} \widetilde{G} H_{\mathbb{C}^{p}}^{2} \subseteq H_{E}^{2} .
$$

It thus follows from Lemma 4.5 and (7.9) that

$$
\Delta_{c} \breve{\theta} I_{p} \widetilde{G} H_{\mathbb{C}^{p}}^{2}=\breve{\theta} I_{E} \Delta_{c} \widetilde{G} H_{\mathbb{C}^{p}}^{2} \subseteq \operatorname{ker} \Delta^{*}=\Delta_{c} H_{\mathbb{C}^{p}}^{2},
$$

which implies $\breve{\theta} I_{p} \widetilde{G} H_{\mathbb{C}^{p}}^{2} \subseteq H_{\mathbb{C}^{p}}^{2}$. We thus have $\breve{\theta} I_{p} \widetilde{G} \in H_{M_{p}}^{\infty}$, so that $\bar{\theta} I_{p} G \in H_{M_{p}}^{\infty}$. Therefore we may write $G=\theta I_{p} G_{1}$ for some $G_{1} \in H_{M_{p}}^{\infty}$. It thus follows that

$$
\theta I_{p}=G\left(\widetilde{\Delta_{c}}\right)^{i}=\theta I_{p} G_{1}\left(\widetilde{\Delta_{c}}\right)^{i}
$$


which gives that $G_{1}\left(\widetilde{\Delta_{c}}\right)^{i}=I_{p}$. Therefore we have

$$
H_{\mathbb{C}^{p}}^{2}=\widetilde{\left(\widetilde{\Delta_{c}}\right)^{i}} \widetilde{G_{1}} H_{\mathbb{C}^{p}}^{2} \subseteq \widetilde{\left(\widetilde{\Delta_{c}}\right)^{i}} H_{\mathbb{C}^{p}}^{2}
$$

which implies that $\widetilde{\left(\widetilde{\Delta_{c}}\right)^{i}}$ is a unitary matrix, and so is $\left(\widetilde{\Delta_{c}}\right)^{i}$. Thus, $\widetilde{\Delta_{c}}$ is an outer function. This completes the proof.

COROLLARY 7.10. If $\Delta$ is an inner matrix function, then $\Delta_{c}^{t}$ is an outer function.

Proof. Immediate from Lemma 7.9

REMARK 7.11. Let $T:=\left.S_{\mathbb{C}^{n}}^{*}\right|_{\mathcal{H}(\Delta)}$ for some non-square inner matrix function $\Delta$. Then Corollary 7.8 shows that if $\Delta=\Omega_{c}$ for an inner matrix function $\Omega$ such that $\breve{\Omega}$ is of bounded type, then $T$ is multiplicity-free. However, the converse is not true in general, i.e., the condition "multiplicity-free" does not guarantee that $\Delta=\Omega_{c}$. To see this, let $\Delta:=[0 z]^{t}$. Then $\Delta$ is inner and $\breve{\Delta}$ is of bounded type. Since $\Delta^{t}=[0 z]$ is not an outer function, it follows from Corollary 7.10 that $\Delta \neq \Omega_{c}$ for any inner matrix function. Let $f:=(a 1)^{t}(\bar{a}$ is not of bounded type). Then $E_{f}^{*}=\mathcal{H}(\Delta)$, so that $T$ is multiplicity-free.

Lemma 7.12. Let $\Delta$ be an inner function and have a meromorphic pseudocontinuation of bounded type in $\mathbb{D}^{e}$. If $\widetilde{\Delta}$ is an outer function and ker $\Delta^{*}=\{0\}$, then $\Delta$ is a unitary operator.

Proof. Let $\Delta$ be an inner function with values in $\mathcal{B}(D, E)$ and have a meromorphic pseudo-continuation of bounded type in $\mathbb{D}^{e}$. Then by Lemma 4.22, $\breve{\Delta}$ is of bounded type. Suppose that $\widetilde{\Delta}$ is an outer function and $\operatorname{ker} \Delta^{*}=\{0\}$. Then by Lemma 4.5. Corollary 4.20 and Lemma $4.22 . \Delta$ is two-sided inner, and so is $\widetilde{\Delta}$. Thus $\Delta$ is a unitary operator, as desired.

The following lemma is a key idea for an answer to Question 1.6.

Lemma 7.13. Let $\Delta$ be an inner function and have a meromorphic pseudocontinuation of bounded type in $\mathbb{D}^{e}$. If $\widetilde{\Delta}$ is an outer function, then

$$
\Delta_{c c}=\Delta \text {. }
$$

Proof. Let $\Delta$ be an inner function with values in $\mathcal{B}(D, E)$ and have a meromorphic pseudo-continuation of bounded type in $\mathbb{D}^{e}$. Also, suppose $\widetilde{\Delta}$ is an outer function. If $\operatorname{ker} \Delta^{*}=\{0\}$, then the result follows at one from Lemma 7.12, Assume that ker $\Delta^{*} \neq\{0\}$. By Lemma 4.22, $\breve{\Delta}$ is of bounded type, so that by Corollary 4.20. $\left[\Delta, \Delta_{c}\right]$ is a two-sided inner function with values in $\mathcal{B}\left(D \oplus D^{\prime}, E\right)$ for some nonzero Hilbert space $D^{\prime}$. We now claim that

$$
\Delta=\Delta_{c c} \Omega \text { for a two-sided inner function } \Omega \text { with values in } \mathcal{B}(D) .
$$

Since $\Delta_{c c}$ is a left inner divisor of $\Delta$ (cf. the Proof of Lemma 7.5), we may write

$$
\Delta=\Delta_{c c} \Omega
$$


for an inner function $\Omega$ with values in $\mathcal{B}\left(D, D^{\prime \prime}\right)$. Assume to the contrary that $\Omega$ is not two-sided inner. Since $\Delta$ has a meromorphic pseudo-continuation of bounded type in $\mathbb{D}^{e}$, it follows from Lemma 4.23 that

$$
\theta H_{E}^{2} \subseteq \operatorname{ker} H_{\Delta^{*}}=\operatorname{ker} H_{\Omega^{*} \Delta_{c c}^{*}}
$$

for some scalar inner function $\theta$. Thus $\Omega^{*} \Delta_{c c}^{*} \theta H_{E}^{2} \subseteq H_{D}^{2}$. In particular, we have

$$
\Omega^{*} \theta H_{D^{\prime \prime}}^{2}=\Omega^{*} \Delta_{c c}^{*} \theta \Delta_{c c} H_{D^{\prime \prime}}^{2} \subseteq H_{D}^{2},
$$

and hence $\theta H_{D^{\prime \prime}}^{2} \subseteq$ ker $H_{\Omega^{*}}$, which implies, by Lemma 4.23, that $\Omega$ has a meromorphic pseudo-continuation of bounded type in $\mathbb{D}^{e}$. Thus by Lemma 4.22, $\breve{\Omega}$ is of bounded type. It thus follows from Lemma 4.5 that

$$
\left[\Omega, \Omega_{c}\right] \text { is two-sided inner, }
$$

where $\Omega_{c}$ is the complementary factor of $\Omega$, with values in $\mathcal{B}\left(D_{1}, D^{\prime \prime}\right)$ for some nonzero Hilbert space $D_{1}$. On the other hand, it follows from (7.12) that for all $f \in H_{D_{1}}^{2}$,

$$
\left[\Delta, \Delta_{c}\right]^{*} \Delta_{c c} \Omega_{c} f=\left[\begin{array}{c}
\Omega^{*} \Omega_{c} f \\
\Delta_{c}^{*} \Delta_{c c} \Omega_{c} f
\end{array}\right]=0,
$$

which implies that $D_{1}=\{0\}$, a contradiction. This proves (7.11). Thus we may write

$$
\widetilde{\Delta}=\widetilde{\Omega} \widetilde{\Delta_{c c}}
$$

for a two-sided inner function $\widetilde{\Omega}$ with values in $\mathcal{B}(D)$. Since $\widetilde{\Delta}$ is an outer function and $\widetilde{\Omega}$ is two-sided inner, it follows from (7.13) that $\widetilde{\Omega}$ is a unitary operator, and so is $\Omega$. This completes the proof.

Lemma 7.13 may fail if the condition " $\Delta$ has a meromorphic pseudo-continuation of bounded type in $\mathbb{D}^{e}$ " is dropped. To see this. let

$$
\Delta:=\left[\begin{array}{l}
f \\
g \\
0
\end{array}\right],
$$

where $f$ and $g$ are given in Example 3.11. Then $\widetilde{\Delta}$ is an outer function. A straightforward calculation shows that

$$
\Delta_{c}=\left[\begin{array}{l}
0 \\
0 \\
1
\end{array}\right] \quad \text { and } \quad \Delta_{c c}=\left[\begin{array}{ll}
1 & 0 \\
0 & 1 \\
0 & 0
\end{array}\right] \neq \Delta .
$$

Note that $\breve{\Delta}$ is not of bounded type. Thus, by Corollary 4.27 $\Delta$ has no meromorphic pseudo-continuation of bounded type in $\mathbb{D}^{e}$.

We are ready to give an answer to Question 1.6.

TheOREM 7.14. (Multiplicity-free model operators) Let $T:=\left.S_{E}^{*}\right|_{\mathcal{H}(\Delta)}$. If $\Delta$ has a meromorphic pseudo-continuation of bounded type in $\mathbb{D}^{e}$ and $\widetilde{\Delta}$ is an outer function, then $T$ is multiplicity-free. 
Proof. Let $T:=\left.S_{E}^{*}\right|_{\mathcal{H}(\Delta)}$. Suppose that $\Delta$ has a meromorphic pseudocontinuation of bounded type in $\mathbb{D}^{e}$ and $\widetilde{\Delta}$ is an outer function. If ker $\Delta^{*}=\{0\}$, then by Lemma 7.12. $\Delta$ is a unitary operator, so that $T$ is multiplicity-free. If instead ker $\Delta^{*} \neq\{0\}$, then by Lemma 7.5, $\Delta_{c}$ has a meromorphic pseudo-continuation of bounded type in $\mathbb{D}^{e}$. Since $\widetilde{\Delta}$ is an outer function it follows from Lemma 7.13 that $\Delta=\Delta_{c c}$. Applying Lemma 7.7 with $\Phi \equiv \Delta_{c}$, we can see that $T$ has a cyclic vector, i.e., $T$ is multiplicity-free.

The following corollary is an immediate result of Theorem 7.14 ,

Corollary 7.15. Let $T:=\left.S_{\mathbb{C}^{n}}^{*}\right|_{\mathcal{H}(\Delta)}$ for an inner matrix function $\Delta$ whose flip $\breve{\Delta}$ is of bounded type. If $\Delta^{t}$ is an outer function, then $T$ is multiplicity-free.

PROOF. This follows from Theorem 7.14 and Corollary 4.27

If $\Delta$ is an inner matrix function then the converse of Lemma 7.13 is also true.

Corollary 7.16. Let $\Delta$ be an inner matrix function whose flip $\breve{\Delta}$ is of bounded type. Then the following are equivalent:

(a) $\Delta^{t}$ is an outer function;

(b) $\widetilde{\Delta}$ is an outer function;

(c) $\Delta_{c c}=\Delta$;

(d) $\Delta=\Omega_{c}$ for some inner matrix function $\Omega$.

Hence, in particular, $\Delta_{c c c}=\Delta_{c}$.

Proof. The implication $(\mathrm{a}) \Rightarrow(\mathrm{b})$ is clear and the implication $(\mathrm{b}) \Rightarrow(\mathrm{c})$ follows from Corollary 4.27 and Lemma 7.13. Also the implication $(\mathrm{c}) \Rightarrow(\mathrm{d})$ is clear and the implication $(\mathrm{d}) \Rightarrow(\mathrm{a})$ follows from Corollary 7.10 . The second assertion follows from the first assertion together with Corollary 4.20 and Corollary 7.10.

\section{$\S 7.3$. A reduction to the case of $C_{0}$-contractions}

On the other hand, the theory of spectral multiplicity for $C_{0}$-operators has been well developed in terms of their characteristic functions (cf. [Ni1, Appendix 1]). However this theory is not applied directly to $C_{0}$-operators, in which cases their characteristic functions need not be two-sided inner. The object of this section is to show that if the characteristic function of a $C_{0}$-operator $T$ has a meromorphic pseudo-continuation of bounded type in $\mathbb{D}^{e}$, then its spectral multiplicity can be computed by that of the $C_{0}$-operator induced by $T$.

We first observe:

LEMmA 7.17. If $\Phi \in L_{\mathcal{B}(D, E)}^{2}$ and $f \in H_{D}^{\infty}$, then $\Phi f \in L_{E}^{2}$.

Proof. Suppose $\Phi \in L_{\mathcal{B}(D, E)}^{2}$ and $f \in H_{D}^{\infty}$. Since $f$ is strongly measurable, there exist countable valued functions $f_{n}=\sum_{k=1}^{\infty} d_{k}^{(n)} \chi_{\sigma_{k}^{(n)}}$ such that $f(z)=\lim _{n} f_{n}(z)$ for almost all $z \in \mathbb{T}$. For all $e \in E$ and $n=1,2,3, \cdots$,

$$
\left\langle\Phi(z) f_{n}(z), e\right\rangle_{E}=\sum_{k=1}^{\infty} \chi_{\sigma_{k}^{(n)}}(z) \cdot\left\langle\Phi(z) d_{k}^{(n)}, e\right\rangle_{D} .
$$


But since $\Phi$ is WOT measurable, by (7.14), $\Phi f_{n}$ is weakly measurable and in turn, $\Phi f: \mathbb{T} \rightarrow E$ is weakly measurable, and hence it is strongly measurable. Observe that

$$
\int_{\mathbb{T}}\|\Phi(z) f(z)\|_{E}^{2} d m(z) \leq\|f\|_{\infty} \int_{\mathbb{T}}\|\Phi(z)\|^{2} d m(z)<\infty,
$$

which implies that $\Phi f \in L_{E}^{2}$. This completes the proof.

Lemma 7.18. Let $\Phi \in L_{\mathcal{B}(D, E)}^{2}$ and let $A: H_{D}^{2} \rightarrow H_{E}^{2}$ be a densely defined operator, with domain $H_{D}^{\infty} \subset H_{D}^{2}$, defined by

$$
A f:=J P_{-}(\Phi f) \quad\left(f \in H_{D}^{\infty}\right) .
$$

Then

$$
\operatorname{ker} A^{*}=\operatorname{ker} H_{\Phi}^{*} \text {. }
$$

Proof. Let $\Phi \in L_{\mathcal{B}(D, E)}^{2} \subseteq L_{s}^{2}(\mathcal{B}(D, E))$. Since the domain of $H_{\Phi}$ is a subset of the domain of $A$, it follows that the domain of $A^{*}$ is a subset of the domain of $H_{\Phi}^{*}$, so that $\operatorname{ker} A^{*} \subseteq \operatorname{ker} H_{\Phi}^{*}$. For the reverse inclusion, suppose $g \in \operatorname{ker} H_{\Phi}^{*}$. Then

$$
\left\langle H_{\Phi} p, g\right\rangle_{L_{E}^{2}}=0 \text { for all } p \in \mathcal{P}_{D} .
$$

Let $f \in H_{D}^{\infty}$ be arbitrary. Then we may write

$$
f(z)=\sum_{k=0}^{\infty} a_{k} z^{k} \quad\left(a_{k} \in D\right)
$$

Let

$$
p_{n}(z):=\sum_{k=0}^{n} a_{k} z^{k} \in \mathcal{P}_{D}
$$

Then it follows from (7.15) that

$$
0=\lim _{n \rightarrow \infty}\left\langle H_{\Phi} p_{n}, g\right\rangle_{L_{E}^{2}}=\lim _{n \rightarrow \infty}\left\langle p_{n}, \Phi^{*} J g\right\rangle_{L_{D}^{2}}=\langle\Phi f, J g\rangle_{L_{E}^{2}}=\langle A f, g\rangle_{L_{E}^{2}},
$$

which implies that $g \in \operatorname{ker} A^{*}$, so that $\operatorname{ker} H_{\Phi}^{*} \subseteq \operatorname{ker} A^{*}$. This completes the proof.

Corollary 7.19. If $\Phi \in H_{\mathcal{B}(D, E)}^{2}$, then

$$
E_{\{\Phi\}}^{*}=\operatorname{cl}\left\{J P_{-}(\bar{z} \breve{\Phi} h): h \in H_{D}^{\infty}\right\} .
$$

Proof. Define $A: H_{D}^{2} \rightarrow H_{E}^{2}$ by $A f:=J P_{-}(\bar{z} \breve{\Phi} h)\left(h \in H_{D}^{\infty}\right)$. By Lemma 7.18, $\operatorname{ker} H_{\bar{z} \breve{\Phi}}^{*}=\operatorname{ker} A^{*}$. By (4.8), we have

$$
E_{\{\Phi\}}^{*}=\operatorname{cl} \operatorname{ran} H_{\bar{z} \breve{\Phi}}=\operatorname{cl} \operatorname{ran} A=\operatorname{cl}\left\{J P_{-}(\bar{z} \breve{\Phi} h): h \in H_{D}^{\infty}\right\} .
$$

We thus have: 
Lemma 7.20. Suppose $\Delta$ is a two-sided inner function and has a meromorphic pseudo-continuation of bounded type in $\mathbb{D}^{e}$. Let $F \equiv\left\{f_{1}, f_{2}, \cdots, f_{p}\right\} \subseteq \mathcal{H}(\Delta)$. Then

$$
E_{F}^{*}=\bigvee\left\{P_{+}\left(\breve{h}_{j} f_{j}\right): h_{j} \in H^{\infty} \cap \mathcal{H}\left(\widetilde{\omega_{\Delta}}\right), j=1,2, \cdots p\right\}
$$

where $\omega_{\Delta}$ is the pseudo-characteristic scalar inner function of $\Delta$.

Proof. Suppose $\Delta$ is a two-sided inner function with values in $\mathcal{B}(E)$ and has a meromorphic pseudo-continuation of bounded type in $\mathbb{D}^{e}$. Let $F \equiv\left\{f_{1}, f_{2}, \cdots, f_{p}\right\} \subseteq$ $\mathcal{H}(\Delta)$. Write $[F]:=\left[\left[f_{1}\right],\left[f_{2}\right], \cdots,\left[f_{p}\right]\right]$ and $\theta:=\omega_{\Delta}$. Since $\left[f_{j}\right] \in H_{\mathcal{B}(\mathbb{C}, E)}^{2}$ for each $j=1,2, \cdots, p$, it is easy to see that $F \in H_{\mathcal{B}\left(\mathbb{C}^{p}, E\right)}^{2}$. We first claim that

$$
E_{F}^{*}=\operatorname{cl}\left\{J P_{-}(\bar{z}[\breve{F}] h): h \in H_{\mathbb{C}^{p}}^{\infty} \cap \mathcal{H}\left(\widetilde{\theta} I_{p}\right)\right\} .
$$

By Corollary 7.19 we have

$$
E_{F}^{*}=\operatorname{cl}\left\{J P_{-}(\bar{z}[\breve{F}] h): h \in H_{\mathbb{C}^{p}}^{\infty}\right\} \supseteq \operatorname{cl}\left\{J P_{-}(\bar{z}[\breve{F}] h): h \in H_{\mathbb{C}^{p}}^{\infty} \cap \mathcal{H}\left(\widetilde{\theta} I_{p}\right)\right\} .
$$

For the reverse inclusion, it suffices to show that

$$
P_{-}(\bar{z}[\breve{F}] \widetilde{\theta} h)=0 \quad \text { for all } h \in H_{\mathbb{C}^{p}}^{\infty} .
$$

By Lemma 4.23, we may write

$$
\Delta=\theta A^{*} \quad \text { for some } A \in H^{\infty}(\mathcal{B}(E)) .
$$

Since $\Delta$ is two-sided inner, it follows that $I_{E}=\Delta \Delta^{*}=A^{*} A$, so that $\theta H_{E}^{2}=$ $\Delta A H_{E}^{2} \subseteq \Delta H_{E}^{2}$. We thus have

$$
\mathcal{H}(\Delta) \subseteq \mathcal{H}\left(\theta I_{E}\right) .
$$

Thus $f_{j} \in \mathcal{H}\left(\theta I_{E}\right)(j=1, \cdots, p)$, so that $\bar{\theta} f_{j} \in L_{E}^{2} \ominus H_{E}^{2}$. Hence for all $h \in H_{\mathbb{C}^{p}}^{\infty}$, by Lemma 7.17 , we have $\bar{\theta}[F] \breve{h} \in L_{E}^{2} \ominus H_{E}^{2}$, so that $\bar{z}[\breve{F}] \widetilde{\theta} h \in H_{E}^{2}$, and hence $P_{-}(\bar{z}[\breve{F}] \tilde{\theta} h)=0$, which gives (7.17). This proves (7.16). Write $h=\left(h_{1}, h_{2}, \cdots h_{p}\right)^{t} \in H_{\mathbb{C}^{p}}^{\infty} \cap \mathcal{H}\left(\widetilde{\theta} I_{p}\right)$, and hence $h_{j} \in H^{\infty} \cap \mathcal{H}(\widetilde{\theta})$. Thus it follows from (17.16) that

$$
\begin{aligned}
E_{F}^{*} & =\operatorname{cl}\left\{J P_{-}(\bar{z}[\breve{F}] h): h \in H_{\mathbb{C}^{p}}^{\infty} \cap \mathcal{H}\left(\widetilde{\theta} I_{p}\right)\right\} \\
& =\bigvee\left\{J P_{-}\left(\bar{z}\left[\breve{f}_{j}\right] h_{j}\right): h_{j} \in H^{\infty} \cap \mathcal{H}(\widetilde{\theta}), j=1,2, \cdots p\right\} \\
& =\bigvee\left\{P_{+}\left(\breve{h}_{j} f_{j}\right): h_{j} \in H^{\infty} \cap \mathcal{H}(\widetilde{\theta}), j=1,2, \cdots p\right\} .
\end{aligned}
$$

This completes the proof.

Lemma 7.21. Let $\Delta$ be an inner function with values in $\mathcal{B}\left(E^{\prime}, E\right)$, with $\operatorname{dim} E^{\prime}<\infty$. If $\widetilde{\Delta}=(\widetilde{\Delta})^{i}(\widetilde{\Delta})^{e}$ is the inner-outer factorization of $\widetilde{\Delta}$, then we have:

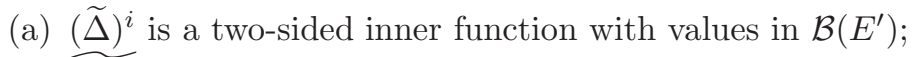

(b) $\widetilde{(\widetilde{\Delta})^{e}}$ is an inner function with values in $\mathcal{B}\left(E^{\prime}, E\right)$. 
Proof. Let $\operatorname{dim} E^{\prime}=r$. Then the inner part $(\widetilde{\Delta})^{i}$ is an $r \times p$ inner matrix function for some $p \leq r$. Thus we have

$$
p=\operatorname{Rank}(\widetilde{\Delta})^{i} \geq \operatorname{Rank} \widetilde{\Delta}=\operatorname{Rank} \Delta=r,
$$

which proves (a). For (b), observe $\Delta=\widetilde{(\widetilde{\Delta})^{e}}(\widetilde{\widetilde{\Delta}})^{i}$. Since $\Delta$ is inner, we have that

$$
I_{r}=\Delta^{*} \Delta={\widetilde{\widetilde{\Delta}})^{i}}^{*} \widetilde{\widetilde{\Delta})^{*}}\left(\widetilde{\widetilde{\Delta})^{e}} \widetilde{\widetilde{\Delta})^{i}} .\right.
$$

But since $(\widetilde{\Delta})^{i}$ is two-sided inner, so is $(\widetilde{\widetilde{\Delta}})^{i}$. Thus it follows that

$$
\widetilde{(\widetilde{\Delta})^{e}} * \widetilde{\widetilde{\Delta})^{e}}=\widetilde{(\widetilde{\Delta})^{i}(\widetilde{\widetilde{\Delta}})^{i}}{ }^{*}=I_{r}
$$

which implies that $\widetilde{(\widetilde{\Delta})^{e}}$ is an inner function. This proves $(\mathrm{b})$.

Lemma 7.22. Suppose $\Delta$ is an inner function with values in $\mathcal{B}\left(E^{\prime}, E\right)$ ), with $\operatorname{dim} E^{\prime}<\infty$ and has a meromorphic pseudo-continuation of bounded type in $\mathbb{D}^{e}$. Write

$$
\Delta_{1}:=\widetilde{(\widetilde{\Delta})^{e}}
$$

Then $\Delta_{1}$ has a meromorphic pseudo-continuation of bounded type in $\mathbb{D}^{e}$.

Proof. Let $\operatorname{dim} E^{\prime}=r$ and let $\widetilde{\Delta}=(\widetilde{\Delta})^{i}(\widetilde{\Delta})^{e}$ be the inner-outer factorization of $\widetilde{\Delta}$. Then

$$
\Delta=\widetilde{(\widetilde{\Delta})^{e}} \widetilde{(\widetilde{\Delta})^{i}} \equiv \Delta_{1} \Delta_{\mathrm{s}} \quad\left(\text { where } \Delta_{1} \equiv \widetilde{(\widetilde{\Delta})^{e}} \text { and } \Delta_{\mathrm{s}} \equiv \widetilde{(\widetilde{\Delta})^{i}}\right) .
$$

By Lemma 7.21, $\Delta_{\mathrm{s}} \in H_{M_{r}}^{\infty}$ is square inner and $\Delta_{1} \in H^{\infty}\left(\mathcal{B}\left(\mathbb{C}^{r}, E\right)\right)$ is inner. Since $\Delta$ has a meromorphic pseudo-continuation of bounded type in $\mathbb{D}^{e}$, it follows from Lemma 4.23 that there exists a scalar inner function $\theta$ such that $\theta H_{E}^{2} \subseteq$ ker $H_{\Delta^{*}}=$ $\operatorname{ker} H_{\Delta_{s}^{*} \Delta_{1}^{*}}$. Thus we have

$$
\Delta_{\mathrm{s}}^{*} \Delta_{1}^{*} \theta H_{E}^{2} \subseteq H_{\mathbb{C}^{r}}^{2}
$$

Since $\Delta_{\mathrm{S}}$ is square inner, it follows from (7.18) that $\Delta_{1}^{*} \theta H_{E}^{2} \subseteq \Delta_{\mathrm{S}} H_{\mathbb{C}^{r}}^{2} \subseteq H_{\mathbb{C}^{r}}^{2}$, so that $\theta H_{E}^{2} \subseteq \operatorname{ker} H_{\Delta_{1}^{*}}$, which implies, by Lemma 4.23, that $\Delta_{1}$ has a meromorphic pseudo-continuation of bounded type in $\mathbb{D}^{e}$. This completes the proof.

Lemma 7.23. Let $\Delta_{1}$ be an inner function with values in $\mathcal{B}(D, E)$ and $\Delta_{2}$ be a two-sided inner function with values in $\mathcal{B}(D)$. Then,

$$
\mathcal{H}\left(\Delta_{1} \Delta_{2}\right)=\mathcal{H}\left(\Delta_{1}\right) \bigoplus \Delta_{1} \mathcal{H}\left(\Delta_{2}\right) .
$$

Proof. The inclusion $\mathcal{H}\left(\Delta_{1}\right) \subseteq \mathcal{H}\left(\Delta_{1} \Delta_{2}\right)$ is clear and by Corollary 3.16 $\Delta_{1} \mathcal{H}\left(\Delta_{2}\right) \subseteq \mathcal{H}\left(\Delta_{1} \Delta_{2}\right)$, which gives $\mathcal{H}\left(\Delta_{1}\right) \bigoplus \Delta_{1} \mathcal{H}\left(\Delta_{2}\right) \subseteq \mathcal{H}\left(\Delta_{1} \Delta_{2}\right)$. For the reverse inclusion, suppose $f \in \mathcal{H}\left(\Delta_{1} \Delta_{2}\right)$. Let $f_{1}:=P_{\mathcal{H}\left(\Delta_{1}\right)}(f)$ and $f_{2}:=f-f_{1}$. Then $f_{2}=\Delta_{1} g$ for some $g \in H_{D}^{2}$. Since $f \in \mathcal{H}\left(\Delta_{1} \Delta_{2}\right)$, it follows from Corollary 3.16 that

$$
\Delta_{2}^{*} \Delta_{1}^{*}\left(f_{1}+\Delta_{1} g\right) \in L_{D}^{2} \ominus H_{D}^{2}
$$

Since $f_{1} \in \mathcal{H}\left(\Delta_{1}\right)$, it follows from Corollary 3.16 that $\Delta_{1}^{*} f_{1} \in L_{D}^{2} \ominus H_{D}^{2}$. Thus by Corollary 3.15, for all $h \in H_{D}^{2}$,

$$
\left\langle\Delta_{2}^{*} \Delta_{1}^{*} f_{1}, h\right\rangle_{L_{D}^{2}}=\left\langle\Delta_{1}^{*} f_{1}, \Delta_{2} h\right\rangle_{L_{D}^{2}}=0
$$


which implies that $\Delta_{2}^{*} \Delta_{1}^{*} f_{1} \in L_{D}^{2} \ominus H_{D}^{2}$. Thus by Corollary 3.16 and (7.19), we have $g \in \mathcal{H}\left(\Delta_{2}\right)$, and hence $f_{2} \in \Delta_{1} \mathcal{H}\left(\Delta_{2}\right)$. Therefore we have $\mathcal{H}\left(\Delta_{1} \Delta_{2}\right) \subseteq$ $\mathcal{H}\left(\Delta_{1}\right) \oplus \Delta_{1} \mathcal{H}\left(\Delta_{2}\right)$. This completes the proof.

We are ready for:

Theorem 7.24. (The spectral multiplicity of model operators) Given an inner function $\Delta$ with values in $\mathcal{B}\left(E^{\prime}, E\right)$, with $\operatorname{dim} E^{\prime}<\infty$, let $T:=\left.S_{E}^{*}\right|_{\mathcal{H}(\Delta)}$. If $\Delta$ has a meromorphic pseudo-continuation of bounded type in $\mathbb{D}^{e}$, then

$$
\mu_{T}=\mu_{T_{s}},
$$

where $T_{s}$ is a $C_{0}$-contraction of the form $T_{s}:=\left.S_{E^{\prime}}^{*}\right|_{\mathcal{H}\left(\Delta_{s}\right)}$ with $\Delta_{s}:=\widetilde{(\widetilde{\Delta})^{i}}$. Hence in particular, $\mu_{T} \leq \operatorname{dim} E^{\prime}$.

Proof. Let $T:=\left.S_{E}^{*}\right|_{\mathcal{H}(\Delta)}$. Suppose $\Delta$ has a meromorphic pseudo-continuation of bounded type in $\mathbb{D}^{e}$. Let $\Delta_{\mathrm{S}} \equiv \widetilde{(\widetilde{\widetilde{\Delta}})^{i}}$ and write

$$
T_{s}:=\left.S_{E^{\prime}}^{*}\right|_{\mathcal{H}\left(\Delta_{\mathrm{s}}\right)} \text {. }
$$

If $\Delta$ is two-sided inner, then $\Delta=\Delta_{\mathrm{S}}$, so that $\mu_{T}=\mu_{T_{\mathrm{s}}}$. Suppose that $\Delta$ is not two-sided inner. Without loss of generality, we may assume that $E^{\prime}=\mathbb{C}^{r}$. By Lemma 7.21, $\Delta_{\mathrm{s}} \in H_{M_{r}}^{\infty}$ is square inner. Thus by (4.16) and Proposition 4.29, we have that $T_{s} \in C_{0}$. We will prove that

$$
\mu_{T}=\mu_{T_{s}} .
$$

Write

$$
\Delta_{1} \equiv \widetilde{(\widetilde{\Delta})^{e}}
$$

Then it follows from Lemma 7.21 and Lemma 7.22 that $\Delta_{1}$ is an inner function having a meromorphic pseudo-continuation of bounded type in $\mathbb{D}^{e}$. Let

$$
\theta:=\omega_{\Delta_{1}} \omega_{\Delta_{\mathrm{s}}} .
$$

Let $p:=\mu_{T_{s}}$. In view of (1.8), we have $p \leq r$. Then there exists a set $F \equiv$ $\left\{f_{1}, f_{2}, \cdots, f_{p}\right\} \subseteq \mathcal{H}\left(\Delta_{\mathrm{s}}\right)$ such that $E_{F}^{*}=\mathcal{H}\left(\Delta_{\mathrm{s}}\right)$. Since by $(\overline{7.22}), \mathcal{H}\left(\widetilde{\omega_{\Delta_{\mathrm{s}}}}\right) \subseteq \mathcal{H}(\widetilde{\theta})$, it follows from Lemma 7.20 that

$$
\mathcal{H}\left(\Delta_{\mathrm{s}}\right)=\bigvee\left\{P_{+}\left(\breve{h}_{j} f_{j}\right): h_{j} \in H^{\infty} \cap \mathcal{H}(\widetilde{\theta}), j=1,2, \cdots p\right\} .
$$

Write

$$
\Omega:=\left(\Delta_{1}\right)_{c} \in H^{\infty}\left(E^{\prime \prime}, E\right) \quad\left(E^{\prime \prime} \text { is a subspace of } E\right) .
$$

Since $\widetilde{\Delta_{1}}$ is outer, it follows from Lemma 7.13 that $\Delta_{1}=\Omega_{c}$. Choose a cyclic vector $g$ of $S_{E^{\prime \prime}}^{*}$. Then it follows from Lemma 7.5, Lemma 7.7 and Lemma 7.20 that

$$
\mathcal{H}\left(\Delta_{1}\right)=E_{\Omega g}^{*}=\operatorname{cl}\left\{P_{+}(\breve{h} \Omega g): h \in H^{\infty}\right\} .
$$

Let

$$
\gamma_{1}:=\theta \Omega g+\Delta_{1} f_{1} \quad \text { and } \quad \gamma_{j}:=\Delta_{1} f_{j} \quad(j=2,3, \cdots, p) .
$$

Now we will show that

$$
\mathcal{H}(\Delta)=\bigvee\left\{P_{+}\left(\breve{\eta}_{j} \gamma_{j}\right): \eta_{j} \in H^{\infty}, j=1,2, \cdots p\right\} .
$$

Let $\xi \in \mathcal{H}(\Delta)$ and $\epsilon>0$ be arbitrary. Then, by Lemma 7.23 we may write

$$
\xi=\xi_{1}+\Delta_{1} \xi_{2} \quad\left(\xi_{1} \in \mathcal{H}\left(\Delta_{1}\right), \xi_{2} \in \mathcal{H}\left(\Delta_{\mathrm{s}}\right)\right) .
$$


By (7.23), there exist $h_{j} \in H^{\infty} \cap \mathcal{H}(\widetilde{\theta})(j=1,2, \cdots, p)$ such that

$$
\left\|\sum_{j=1}^{p} P_{+}\left(\breve{h}_{j} f_{j}\right)-\xi_{2}\right\|_{L_{\mathbb{C}^{r}}^{2}}<\frac{\epsilon}{2}
$$

For each $j=1,2, \cdots, p$, observe that

$$
\begin{aligned}
P_{+}\left(\breve{h}_{j} \Delta_{1} f_{j}\right) & =P_{+}\left(\Delta_{1} \breve{h}_{j} f_{j}\right) \\
& =\Delta_{1} P_{+}\left(\breve{h}_{j} f_{j}\right)+P_{+}\left(\Delta_{1} P_{-}\left(\breve{h}_{j} f_{j}\right)\right),
\end{aligned}
$$

and

$$
\Delta_{1} P_{+}\left(\breve{h}_{j} f_{j}\right) \in \Delta_{1} \mathcal{H}\left(\Delta_{\mathrm{s}}\right) \quad \text { and } \quad P_{+}\left(\Delta_{1} P_{-}\left(\breve{h}_{j} f_{j}\right)\right) \in \mathcal{H}\left(\Delta_{1}\right) .
$$

Since $\operatorname{ker}(\theta \Omega)^{*}=\operatorname{ker} \Omega^{*}$, we have $(\theta \Omega)_{c}=\Omega_{c}$. Thus by (7.24), $P_{+}\left(\breve{h}_{1} \theta \Omega g\right)$ belongs to $\mathcal{H}\left(\Delta_{1}\right)$. Thus it follows from (7.28) that

$$
\xi_{0} \equiv \xi_{1}-\sum_{j=1}^{p} P_{+}\left(\Delta_{1} P_{-}\left(\breve{h}_{j} f_{j}\right)\right)-P_{+}\left(\breve{h}_{1} \theta \Omega g\right) \in \mathcal{H}\left(\Delta_{1}\right) .
$$

Thus by (7.24), there exists $h_{0} \in H^{\infty}$ such that

$$
\left\|P_{+}\left(\breve{h}_{0} \Omega g\right)-\xi_{0}\right\|_{L_{E}^{2}}<\frac{\epsilon}{2} \text {. }
$$

Let

$$
\eta_{1}:=\widetilde{\theta} h_{0}+h_{1} \quad \text { and } \quad \eta_{j}:=h_{j} \quad(j=2,3, \cdots, p) .
$$

It follows from Lemma 4.23 that

$$
\Delta_{\mathrm{s}}=\omega_{\Delta_{\mathrm{s}}} A^{*} \quad\left(A \in H_{M_{r}}^{\infty}\right) .
$$

It thus follows that $\overline{\omega_{\Delta_{\mathrm{s}}}} f_{1}=A^{*} \Delta_{\mathrm{s}}^{*} f_{1} \in L_{\mathbb{C}^{r}}^{2} \ominus H_{\mathbb{C}^{r}}^{2}$. Thus we have

$$
\bar{\theta} \breve{h_{0}} \Delta_{1} f_{1}=\breve{h}_{0} \overline{\omega_{\Delta_{1}}} \Delta_{1} \overline{\omega_{\Delta_{\mathrm{s}}}} f_{1} \in L_{E}^{2} \ominus H_{E}^{2} \text {, }
$$

which implies $P_{+}\left(\bar{\theta} \breve{h} \Delta_{0} \Delta_{1}\right)=0$. Therefore,

$$
\begin{aligned}
\sum_{j=1}^{p} P_{+}\left(\breve{\eta}_{j} \gamma_{j}\right) & =P_{+}\left(\left(\bar{\theta} \breve{h}_{0}+\breve{h}_{1}\right)\left(\theta \Omega g+\Delta_{1} f_{1}\right)\right)+\sum_{j=2}^{p} P_{+}\left(\breve{h}_{j} \Delta_{1} f_{j}\right) \\
& =P_{+}\left(\breve{h}_{0} \Omega g\right)+P_{+}\left(\breve{h}_{1} \theta \Omega g\right)+\sum_{j=1}^{p} P_{+}\left(\breve{h}_{j} \Delta_{1} f_{j}\right) .
\end{aligned}
$$

Since $\Delta_{1}$ is inner, it follows from (7.26), (7.27) and (7.29) that

$$
\begin{aligned}
\left\|\sum_{j=1}^{p} P_{+}\left(\breve{\eta}_{j} \gamma_{j}\right)-\xi\right\|_{L_{E}^{2}} & \leq\left\|P_{+}\left(\breve{h}_{0} \Omega g\right)-\xi_{0}\right\|_{L_{E}^{2}}+\left\|\sum_{j=1}^{p} \Delta_{1} P_{+}\left(\breve{h}_{j} f_{j}\right)-\Delta_{1} \xi_{2}\right\|_{L_{E}^{2}} \\
& <\epsilon .
\end{aligned}
$$

This proves (7.25). Let $\Gamma:=\left\{\gamma_{1}, \gamma_{2}, \cdots, \gamma_{p}\right\}$. It thus follows from Lemma 7.20 and (17.25) that

$$
E_{\Gamma}^{*}=\bigvee\left\{P_{+}\left(\breve{\eta}_{j} \gamma_{j}\right): \eta_{j} \in H^{\infty}, j=1,2, \cdots p\right\}=\mathcal{H}(\Delta)
$$


which implies that $\mu_{T} \leq \mu_{T_{s}}$. For the reverse inequality, let $q \equiv \mu_{T}<\infty$. Then there exists a set $F \equiv\left\{f_{1}, f_{2}, \cdots, f_{q}\right\} \subseteq \mathcal{H}(\Delta)$ such that $E_{F}^{*}=\mathcal{H}(\Delta)$. For each $j=1,2 \cdots q$, by Lemma 7.23, we can write

$$
f_{j}=g_{j}+\Delta_{1} \gamma_{j} \quad\left(g_{j} \in \mathcal{H}\left(\Delta_{1}\right), \gamma_{j} \in \mathcal{H}\left(\Delta_{\mathrm{s}}\right)\right) .
$$

Now we will show that

$$
E_{\Gamma}^{*}=\mathcal{H}\left(\Delta_{\mathrm{s}}\right) \quad\left(\Gamma \equiv\left\{\gamma_{j}: j=1,2, \cdots, q\right\}\right) .
$$

Clearly, $E_{\Gamma}^{*} \subseteq \mathcal{H}\left(\Delta_{\mathrm{s}}\right)$. On the other hand, since $E_{F}^{*}=\mathcal{H}(\Delta)$ and $\mathcal{H}\left(\Delta_{1}\right)$ is an invariant subspace for $S_{E}^{*}$, it follows from Lemma 7.20 (17.27) and (77.28) that

$$
\begin{aligned}
\Delta_{1} \mathcal{H}\left(\Delta_{\mathrm{S}}\right) & =\bigvee\left\{P_{\Delta_{1} \mathcal{H}\left(\Delta_{s}\right)}\left(S_{E}^{* n} \Delta_{1} \gamma_{j}\right): j=1,2, \cdots q, n=0,1,2 \cdots\right\} \\
& =\bigvee\left\{P_{\Delta_{1} \mathcal{H}\left(\Delta_{s}\right)}\left(\breve{h}_{j} \Delta_{1} \gamma_{j}\right): h_{j} \in H^{\infty}, j=1,2, \cdots p\right\} \\
& =\bigvee\left\{\Delta_{1} P_{+}\left(\breve{h}_{j} \gamma_{j}\right): h_{j} \in H^{\infty}, j=1,2, \cdots p\right\} \\
& =\Delta_{1} E_{\Gamma}^{*} .
\end{aligned}
$$

This proves (7.30). Thus we have that $\mu_{T_{s}} \leq q=\mu_{T}$. This proves (7.21). The last assertion follows at once from (1.8) since $\Delta_{\mathrm{S}}$ is square-inner. This completes the proof.

Corollary 7.25. Suppose $\Delta$ is an $n \times r$ inner matrix function whose flip $\breve{\Delta}$ is of bounded type. If $T:=\left.S_{E}^{*}\right|_{\mathcal{H}(\Delta)}$, then $\mu_{T} \leq r$.

PROOF. It follows from Corollary 4.27 and Theorem 7.24 


\section{CHAPTER 8}

\section{Miscellanea}

In this chapter, by using the preceding results, we analyze left and right coprimeness, the model operator, and an interpolation problem for operator-valued functions.

\section{$\S$ 8.1. Left and right coprimeness}

In this section we consider conditions for the equivalence of left coprime-ness and right coprime-ness.

If $\delta$ is a scalar inner function, a function $A \in H^{\infty}(\mathcal{B}(E))$ is said to have a scalar inner multiple $\delta$ if there exists a function $G \in H^{\infty}(\mathcal{B}(E))$ such that

$$
G A=A G=\delta I_{E} .
$$

We write $\operatorname{mul}(A)$ for the set of all scalar inner multiples of $A$, and we define

$$
m_{A}:=\text { g.c.d. }\{\delta: \delta \in \operatorname{mul}(A)\} \text {. }
$$

We note that if $\Delta$ is a two-sided inner function then by Lemma 4.23 and (7.3), the following are equivalent:

(a) $\Delta$ has a meromorphic pseudo-continuation of bounded type in $\mathbb{D}^{e}$;

(b) $\Delta$ has a scalar inner multiple.

Thus if $\Delta \in H^{\infty}(\mathcal{B}(E))$ is two-sided inner and has a scalar multiple, then $m_{\Delta}$ defined in (8.1) coincides with the characteristic function of $\Delta$. This justifies the use of the notation $m_{A}$ for (8.1).

On the other hand, we may ask:

Question 8.1. If $A \in H^{\infty}(\mathcal{B}(D, E))$ has a scalar inner multiple, does it follow that $m_{A} \in \operatorname{mul}(A)$ ?

If $A$ is two-sided inner with values in $\mathcal{B}(E)$, then the answer to Question 8.1 is affirmative: indeed, by (7.3),

$$
m_{A} H_{E}^{2}=\bigvee\left\{\delta H_{E}^{2}: \delta \in \operatorname{mul}(A)\right\} \subseteq \operatorname{ker} H_{A^{*}},
$$

which implies, again by (7.3), that

$$
m_{A} \in \operatorname{mul}(A) .
$$

Lemma 8.2. If $A \in H^{\infty}(\mathcal{B}(E))$ is an outer function having a scalar inner multiple, then $1 \in \operatorname{mul}(A)$, i.e., $A$ is invertible in $H^{\infty}(\mathcal{B}(E))$. 
Proof. Suppose that $A \in H^{\infty}(\mathcal{B}(E))$ is an outer function having a scalar inner multiple $\delta$. Then

$$
A G=G A=\delta I_{E} \quad \text { for some } G \in H^{\infty}(\mathcal{B}(E)) .
$$

We claim that

$$
A H_{E}^{2}=\operatorname{cl} A H_{E}^{2} .
$$

To see this, suppose $f \in \operatorname{cl} A H_{E}^{2}$. Then there exists a sequence $\left(g_{n}\right)$ in $H_{E}^{2}$ such that $\left\|A g_{n}-f\right\|_{L_{E}^{2}} \longrightarrow 0$. Thus we have that

$$
\left\|G A g_{n}-G f\right\|_{L_{E}^{2}} \leq\|G\|_{\infty}\left\|A g_{n}-f\right\|_{L_{E}^{2}} \longrightarrow 0 .
$$

It thus follows from (8.3) and (8.5) that

$$
\left\|g_{n}-\bar{\delta} G f\right\|_{L_{E}^{2}}=\left\|\delta g_{n}-G f\right\|_{L_{E}^{2}}=\left\|G A g_{n}-G f\right\|_{L_{E}^{2}} \longrightarrow 0
$$

But since $H_{E}^{2}$ is a closed subspace of $L_{E}^{2}$, we have $g \equiv \bar{\delta} G f \in H_{E}^{2}$. Since $A \in$ $H^{\infty}(\mathcal{B}(E))$, it follows that

$$
\left\|A g_{n}-A g\right\|_{L_{E}^{2}} \leq\|A\|_{\infty}\left\|g_{n}-g\right\|_{L_{E}^{2}} \longrightarrow 0,
$$

which implies that $f=A g \in A H_{E}^{2}$. This proves (8.4). Since $A$ is an outer function, it follows from (8.4) that

$$
A H_{E}^{2}=\operatorname{cl} A H_{E}^{2} \supseteq \operatorname{cl} A \mathcal{P}_{E}=H_{E}^{2},
$$

so that

$$
A H_{E}^{2}=H_{E}^{2}
$$

We thus have that

$$
H_{E}^{2}=(\bar{\delta} G) A H_{E}^{2}=\bar{\delta} G H_{E}^{2},
$$

which implies that $G_{1}:=\bar{\delta} G \in H^{\infty}(\mathcal{B}(E))$. It thus follows from (8.3) that

$$
A G_{1}=G_{1} A=I_{E}
$$

which gives the result.

We are tempted to guess that (8.6) holds for every outer function $A$ in $H^{\infty}(\mathcal{B}(E))$. However, the following example shows that this is not such a case.

ExAmple 8.3. Let $A:=\operatorname{diag}\left(\frac{1}{n}\right) \in H^{\infty}\left(\mathcal{B}\left(\ell^{2}\right)\right)$. Then $\left(1, \frac{1}{2}, \frac{1}{3}, \cdots\right)^{t} \notin A H_{\ell^{2}}^{2}$, so that

$$
A H_{\ell^{2}}^{2} \neq H_{\ell^{2}}^{2} \text {. }
$$

Now we will show that $A$ is an outer function. Let $f \in H_{\ell^{2}}^{2}$ and $\epsilon>0$ be arbitrary. Then we may write

$$
f(z)=\sum_{n=0}^{\infty} c_{n} z^{n} \quad\left(c_{n} \in \ell^{2}\right) .
$$

Thus there exists $M>0$ such that

$$
\left\|\sum_{n=M}^{\infty} c_{n} z^{n}\right\|_{L_{\ell^{2}}^{2}}<\frac{\epsilon}{2} .
$$

Write

$$
f_{1}(z):=\sum_{n=0}^{M-1} c_{n} z^{n} \quad \text { and } \quad f_{2}(z):=\sum_{n=M}^{\infty} c_{n} z^{n} .
$$


For each $n=0,1,2, \cdots, M-1$, write

$$
c_{n}=\left(a_{n}^{(1)}, a_{n}^{(2)}, a_{n}^{(3)}, \cdots\right)^{t} \quad\left(a_{n} \in \mathbb{C}\right) .
$$

Then there exists $N>0$ such that

$$
\left(\sum_{k=N+1}^{\infty}\left|a_{n}^{(k)}\right|^{2}\right)^{\frac{1}{2}}<\frac{\epsilon}{2 M} \text { for each } n=0,1,2, \cdots, M-1 .
$$

Let

$$
p(z):=\sum_{n=0}^{M-1} b_{n} z^{n}, \quad\left(b_{n}:=\left(a_{n}^{(1)}, 2 a_{n}^{(2)}, 3 a_{n}^{(3)}, \cdots N a_{n}^{(N)}, 0,0, \cdots\right)^{t}\right) .
$$

Then it follows from (8.7) and (8.8) that

$$
\begin{aligned}
\|f(z)-(A p)(z)\|_{L_{\ell^{2}}^{2}} & =\left\|f_{1}(z)+f_{2}(z)-(A p)(z)\right\|_{L_{\ell^{2}}^{2}} \\
& =\left.\left\|f_{1}(z)-(A p)(z)\right\|\right|_{L_{\ell^{2}}^{2}}+\left\|f_{2}(z)\right\|_{L_{\ell^{2}}^{2}} \\
& <\frac{\epsilon}{2 M} M+\frac{\epsilon}{2}=\epsilon,
\end{aligned}
$$

which implies that $A$ is an outer function.

LEMmA 8.4. If $A \in H^{\infty}(\mathcal{B}(E))$ has a scalar inner multiple, then

(a) $A^{i}$ is two-sided inner and has a scalar inner multiple with $\operatorname{mul}(A) \subseteq$ $\operatorname{mul}\left(A^{i}\right)$

(b) $1 \in \operatorname{mul}\left(A^{e}\right)$.

Proof. Suppose that $A \in H^{\infty}(\mathcal{B}(E))$ has a scalar inner multiple $\delta$, i.e., $\delta \in$ $\operatorname{mul}(A)$. Then there exist a function $G \in H^{\infty}(\mathcal{B}(E))$ such that

$$
A G=G A=\delta I_{E} \text {. }
$$

Thus $A(z)$ and $G(z)$ are invertible for almost all $z \in \mathbb{T}$. Write

$$
A=A^{i} A^{e} \quad \text { (inner-outer factorization). }
$$

Since $A(z)$ is invertible for almost all $z \in \mathbb{T}, A^{i}(z)$ is onto for almost all $z \in \mathbb{T}$, so that $A^{i}$ is two-sided inner. Also $A^{e}(z)$ is injective for almost all $z \in \mathbb{T}$. By (8.9),

$$
A^{e}(z) G(z)=\delta(z)\left(A^{i}(z)\right)^{*},
$$

which implies that $A^{e}(z)$ is onto, and hence invertible for almost all $z \in \mathbb{T}$. Thus $\left(A^{e} G\right)(z)$ is invertible for almost all $z \in \mathbb{T}$. We thus have that

$$
A^{i}\left(A^{e} G\right)=A G=\delta I_{E}=\left(A^{e} G\right) A^{i},
$$

which implies that $A^{i}$ has a scalar inner multiple $\delta$, i.e., $\delta \in \operatorname{mul}\left(A^{i}\right)$. This proves (a). Also observe that

$$
\left(G A^{i}\right) A^{e}=\delta I_{E}=A^{e}\left(G A^{i}\right),
$$

which implies that $A^{e}$ has a scalar inner multiple. Thus by Lemma $8.2,1 \in$ $\operatorname{mul}\left(A^{e}\right)$. This proves (b).

Lemma 8.5. If $A \in H^{\infty}(\mathcal{B}(E))$ has a scalar inner multiple, then

$$
\operatorname{mul}(A)=\operatorname{mul}\left(A^{i}\right) \text {. }
$$


Proof. In view of Lemma 8.4 (a), it suffices to show that $\operatorname{mul}\left(A^{i}\right) \subseteq \operatorname{mul}(A)$. To see this, let $\delta \in \operatorname{mul}\left(A^{i}\right)$. Then

$$
A^{i} G=G A^{i}=\delta I_{E} \quad \text { for some } G \in H^{\infty}(\mathcal{B}(E)) .
$$

Put $G_{0}:=\left(A^{e}\right)^{-1} G$. Then by Lemma 8.4. $G_{0} \in H^{\infty}(\mathcal{B}(E))$ and

$$
A G_{0}=A^{i} A^{e}\left(A^{e}\right)^{-1} G=\delta I_{E} .
$$

But since $A$ has a scalar inner multiple, $A(z)$ is invertible for almost all $z \in \mathbb{T}$. Thus we have $\delta \in \operatorname{mul}(A)$. This proves $\operatorname{mul}\left(A^{i}\right) \subseteq \operatorname{mul}(A)$. This completes the proof.

The following corollary gives an affirmative answer to Question 8.1

Corollary 8.6. If $A \in H^{\infty}(\mathcal{B}(E))$ has a scalar inner multiple then

$$
m_{A} \in \operatorname{mul}(A) .
$$

Proof. By Lemma 8.4, $A^{i}$ is two-sided inner. By (8.2), $m_{A^{i}} \in \operatorname{mul}\left(A^{i}\right)$. Thus it follows from Lemma 8.5 that

$$
m_{A}=m_{A^{i}} \in \operatorname{mul}\left(A^{i}\right)=\operatorname{mul}(A) .
$$

The following lemma is elementary.

Lemma 8.7. Let $E$ be a complex Hilbert space. If $\theta$ and $\delta$ are scalar inner functions, then

$$
\text { left-g.c.d. }\left\{\theta I_{E}, \delta I_{E}\right\}=\text { g.c.d. }\{\theta, \delta\} I_{E} \text {. }
$$

Proof. Let

$$
\Omega:=\text { left-g.c.d. }\left\{\theta I_{E}, \delta I_{E}\right\} \quad \text { and } \quad \omega:=\text { g.c.d. }\{\theta, \delta\} .
$$

Then we can write

$$
\theta=\omega \theta_{1} \quad \text { and } \quad \delta=\omega \delta_{1}
$$

where $\theta_{1}$ and $\delta_{1}$ are coprime inner functions. Thus we have

$$
\Omega H_{E}^{2}=\theta H_{E}^{2} \bigvee \delta H_{E}^{2}=\omega \theta_{1} H_{E}^{2} \bigvee \omega \delta_{1} H_{E}^{2}=\omega\left(\theta_{1} H_{E}^{2} \bigvee \delta_{1} H_{E}^{2}\right)=\omega H_{E}^{2},
$$

which implies that $\Omega=\omega I_{E}$. This completes the proof.

Lemma 8.8. Let $A \in H^{\infty}(\mathcal{B}(E))$ have a scalar inner multiple and $\theta$ be a scalar inner function. Suppose that $m_{A}$ is not an inner divisor of $\theta$. If $\delta_{0} \in \operatorname{mul}(A)$ is such that $A$ and $\omega I_{E} \equiv$ g.c.d. $\left\{\theta, \delta_{0}\right\} I_{E}$ are left coprime, then $\delta_{0} \bar{\omega} \in \operatorname{mul}(A)$.

Proof. Let $A \in H^{\infty}(\mathcal{B}(E))$ have a scalar inner multiple and $\theta$ be a scalar inner function. Suppose that $m_{A}$ is not an inner divisor of $\theta$. Then we should have $1 \notin \operatorname{mul}(A)$. Thus, by Lemma 8.2, $A$ is not an outer function, so that $A^{i}$ is not a unitary operator. Let $\delta_{0} \in \operatorname{mul}(A)$ be such that $A$ and $\omega I_{E} \equiv$ g.c.d. $\left\{\theta, \delta_{0}\right\} I_{E}$ are left coprime. Then, by Lemma 8.7, we may write

$$
\theta=\omega \theta_{1} \quad \text { and } \quad \delta_{0}=\omega \delta_{1},
$$


where $\theta_{1}$ and $\delta_{1}$ are coprime scalar inner functions. On the other hand, since $\delta_{0} \in \operatorname{mul}(A)$, we have that

$$
\delta_{0} I_{E}=G A=A G \text { for some } G \in H^{\infty}(\mathcal{B}(E)) .
$$

Thus by (8.10) and (8.11), we have that

$$
G\left(\bar{\omega} I_{E}\right) A=\left(\bar{\omega} I_{E}\right) G A=\delta_{1} I_{E} \in H^{\infty}(\mathcal{B}(E)),
$$

which implies that

$$
A H_{E}^{2} \subseteq \operatorname{ker} H_{G\left(\bar{\omega} I_{E}\right)} \equiv \Theta H_{E^{\prime}}^{2} .
$$

Thus $\Theta$ is a left inner divisor of $A$. Since also $\omega H_{E}^{2} \subseteq \operatorname{ker} H_{G \bar{\omega} I_{E}}=\Theta H_{E^{\prime}}^{2}, \Theta$ is a left inner divisor of $\omega I_{E}$. Thus $\Theta$ is a common left inner divisor of $A$ and $\omega I_{E}$, so that, by our assumption, $\Theta$ is a unitary operator. Thus

$$
\operatorname{ker} H_{G \bar{\omega} I_{E}}=\Theta H_{E^{\prime}}^{2}=H_{E}^{2},
$$

which implies that $\bar{\omega} I_{E} G \in H^{\infty}(\mathcal{B}(E))$. On the other hand, by (8.10) and (8.11), we have

$$
\delta_{1} I_{E}=\left(\bar{\omega} \delta_{0}\right) I_{E}=\left(\bar{\omega} I_{E} G\right) A=A\left(\bar{\omega} I_{E} G\right),
$$

which implies that $\delta_{1}=\delta_{0} \bar{\omega} \in \operatorname{mul}(A)$. This completes the proof.

We then have:

Theorem 8.9. Let $A \in H^{\infty}(\mathcal{B}(E))$ and $\theta$ be a scalar inner function. If $A$ has a scalar inner multiple, then the following are equivalent:

(a) $\theta$ and $m_{A}$ are coprime;

(b) $\theta I_{E}$ and $A$ are left coprime;

(c) $\theta I_{E}$ and $A$ are right coprime.

Proof. Let $A \in H^{\infty}(\mathcal{B}(E))$ have a scalar inner multiple. Write

$$
A=A^{i} A^{e} \quad \text { (inner-outer factorization). }
$$

(a) $\Rightarrow(\mathrm{b})$ : Suppose that $\theta I_{E}$ and $A$ are not left coprime. Then

$$
\theta H_{E}^{2} \bigvee A^{i} H_{E}^{2} \neq H_{E}^{2}
$$

By Corollary 8.6 there exists $G \in H^{\infty}(\mathcal{B}(E))$ such that $G A=A G=m_{A} I_{E}$. Thus we have that

$$
\text { left-g.c.d. }\left\{\theta I_{E}, m_{A} I_{E}\right\} H_{E}^{2}=\theta H_{E}^{2} \bigvee A G H_{E}^{2} \subseteq \theta H_{E}^{2} \bigvee A^{i} H_{E}^{2} \neq H_{E}^{2} \text {, }
$$

which implies that $\theta I_{E}$ and $m_{A} I_{E}$ are not left coprime. Thus by Lemma 8.7, $\theta$ and $m_{A}$ are not coprime.

(b) $\Rightarrow$ (a): Suppose that $\theta$ and $m_{A}$ are not coprime. If $m_{A}$ is an inner divisor of $\theta$, then by Corollary 8.6 and Lemma 8.7, we may write

$$
\theta I_{E}=m_{A} \theta_{1} I_{E}=A^{i} A^{e} G \theta_{1} I_{E} \quad\left(G \in H^{\infty}(\mathcal{B}(E)), \theta_{1} \text { is a scalar inner }\right) .
$$

Thus, $A^{i}$ is a common left inner divisor of $\theta I_{E}$ and $A$. If $A^{i}$ is a unitary operator, then $A$ is an outer function. It thus follows from Lemma 8.2 that $m_{A}=1$, so that $\theta$ and $m_{A}$ are coprime, a contradiction. Therefore $A^{i}$ is not a unitary operator, 
8. MISCELLANEA

and hence $\theta I_{E}$ and $A$ are not left coprime. Suppose instead that $m_{A}$ is not an inner divisor of $\theta$. Write $\omega \equiv$ g.c.d. $\left\{\theta, m_{A}\right\} \neq 1$. We then claim that

$$
A \text { and } \omega I_{E} \text { are not left coprime. }
$$

Towards (8.13), we assume to the contrary that $A$ and $\omega I_{E}$ are left coprime. Then it follows from Corollary 8.6 and Lemma 8.8 that $\bar{\omega} m_{A} \in \operatorname{mul}(A)$, which contradicts the definition of $m_{A}$. This proves (8.13). But since $\omega$ is an inner divisor of $\theta$, it follows from Lemma 8.7 that $A$ and $\theta I_{E}$ is not left coprime.

(b) $\Leftrightarrow(\mathrm{c})$. Since $\delta \in \operatorname{mul}(A)$ if and only if $\widetilde{\delta} \in \operatorname{mul}(\widetilde{A})$, it follows that $\widetilde{m_{A}}=m_{\widetilde{A}}$. It thus follows from (a) $\Leftrightarrow(\mathrm{b})$. This completes the proof.

Corollary 8.10. Let $\Delta$ be an inner function with values in $\mathcal{B}(D, E)$ and $\theta$ be a scalar inner function. If $\Delta$ has a meromorphic pseudo-continuation of bounded type in $\mathbb{D}^{e}$, then the following are equivalent:

(a) $\theta$ and $\omega_{\Delta}$ are coprime;

(b) $\theta I_{E}$ and $\left[\Delta, \Delta_{c}\right]$ are left coprime;

(c) $\theta I_{E}$ and $\left[\Delta, \Delta_{c}\right]$ are right coprime.

Proof. Suppose that $\Delta$ has a meromorphic pseudo-continuation of bounded type in $\mathbb{D}^{e}$. Then by Lemma 4.22, $\breve{\Delta}$ is of bounded type, so that by Corollary 4.20 . $\left[\Delta, \Delta_{c}\right]$ is two-sided inner. Thus the result follows from Theorem 8.9 and Lemma 7.5 .

EXAMPLE 8.11. Let

$$
\Delta:=\left[\begin{array}{cc}
b_{\alpha} & 0 \\
0 & b_{\beta} \\
0 & 0
\end{array}\right] \quad(\alpha \neq 0, \beta \neq 0) .
$$

Then $z I_{3}$ and $\Delta$ are not left coprime because $z H_{\mathbb{C}^{3}}^{2} \bigvee \Delta H_{\mathbb{C}^{2}}^{2} \neq H_{\mathbb{C}^{3}}^{2}$. But $z I_{3}$ and $\left[\Delta, \Delta_{c}\right]$ are left coprime, so that, by Corollary 8.10, $z$ and $\omega_{\Delta}$ are coprime. Indeed, we note that ker $H_{\Delta^{*}}=\left[\Delta, \Delta_{c}\right] H_{\mathbb{C}^{3}}^{2}$, and hence $\omega_{\Delta}=b_{\alpha} b_{\beta}$.

The following example shows that if the condition " $A$ has a scalar inner multiple" is dropped in Theorem 8.9, then Theorem 8.9 may fail.

EXAMPLE 8.12. Let

$$
\Delta(z)=S_{E} \quad\left(E=\ell^{2}\left(\mathbb{Z}_{+}\right)\right)
$$

Then $\Delta$ is an inner function (not two-sided inner, an isometric operator) with values in $\mathcal{B}(E)$. For $f \in H_{E}^{2}$, we can write

$$
f(z)=\sum_{n=0}^{\infty} a_{n} z^{n} \quad\left(a_{n} \in E\right) .
$$

We thus have that

$$
(\widetilde{\Delta} f)(z)=S^{*}\left(\sum_{n=0}^{\infty} a_{n} z^{n}\right)=\sum_{n=0}^{\infty}\left(S^{*} a_{n}\right) z^{n}
$$


Thus $\widetilde{\Delta} H_{E}^{2}=H_{E}^{2}$, so that $\Delta$ and $\theta I_{E}$ are right coprime for all scalar inner function $\theta$. Let $\theta(z)=z \theta_{1}$ ( $\theta_{1}$ a scalar inner $)$. Then

$$
(\Delta f)(z)=S\left(\sum_{n=0}^{\infty} a_{n} z^{n}\right)=\sum_{n=0}^{\infty}\left(S a_{n}\right) z^{n} .
$$

We thus have

$$
\Delta H_{E}^{2} \bigvee \theta H_{E}^{2}=\Delta H_{E}^{2} \bigvee z \theta_{1} H_{E}^{2} \subseteq \Delta H_{E}^{2} \bigvee z H_{E}^{2} \neq H_{E}^{2},
$$

which implies that $\theta I_{E}$ and $\Delta$ are not left coprime. Note that $\Delta$ has no scalar inner multiple.

On the other hand, since $\operatorname{ker} H_{\Delta^{*}}=H_{E}^{2}$, we have $\omega_{\Delta}=1$. Thus, it follows from Corollary 8.10 that $\theta I_{E}$ and $\left[\Delta, \Delta_{c}\right]$ are left (and right) coprime for all scalar inner function $\theta$.

LEMma 8.13. If $\Delta \in H_{M_{n}}^{\infty}$ is an inner function then

$$
\theta \text { and } m_{\Delta} \text { are coprime } \Longleftrightarrow \theta \text { and } \operatorname{det} \Delta \text { are coprime. }
$$

Proof. If $\Delta \in H_{M_{n}}^{\infty}$ is inner, then $m_{\Delta} \in \operatorname{mul}(\Delta)$, so that we may write

$$
m_{\Delta} I_{n}=\Delta G \text { for some inner function } G \in H_{M_{n}}^{\infty} .
$$

Thus, $\operatorname{det} \Delta \operatorname{det} G=m_{\Delta}^{n}$. If $\theta$ and $m_{\Delta}$ are coprime, then $\theta$ and $m_{\Delta}^{n}$ are coprime, so that $\theta$ and $\operatorname{det} \Delta$ are coprime. Conversely, suppose that $\theta$ and $\operatorname{det} \Delta$ are coprime. Since $(\operatorname{det} \Delta) I_{n}=(\operatorname{adj} \Delta) \Delta$, it follows that $\operatorname{det} \Delta \in \operatorname{mul}(\Delta)$. Thus, $m_{\Delta}$ is an inner divisor of $\operatorname{det} \Delta$, and hence $\theta$ and $m_{\Delta}$ are coprime. This proves (8.14).

We can recapture [CHL3, Theorem 4.16].

Corollary 8.14. Let $A \in H_{M_{n}}^{2}$ and $\theta$ be a scalar inner function. Then the following are equivalent:

(a) $\theta$ and $\operatorname{det} A$ are coprime;

(b) $\theta I_{n}$ and $A$ are left coprime;

(c) $\theta I_{n}$ and $A$ are right coprime.

Proof. If $\Delta \in H_{M_{n}}^{\infty}$ is inner then by Theorem 8.9 and Lemma 8.13, we have

$$
\theta I_{n} \text { and } \Delta \text { are left coprime } \Longleftrightarrow \theta \text { and } \operatorname{det} \Delta \text { are coprime. }
$$

We now write

$$
A=A^{i} A^{e} \quad \text { (inner-outer factorization). }
$$

Now we will show that if (b) or (c) holds, then $A^{i}$ is two-sided inner: indeed if (b) or (c) holds, then by CHL3, Lemma 4.15], $\operatorname{det} A \neq 0$, so that $A(z)$ is invertible, and hence $A^{i}(z)$ is onto for almost all $z \in \mathbb{T}$. Thus $A^{i}$ is two-sided inner. Then by the Helson-Lowdenslager Theorem (cf. [Ni1, p.22]) we have that

$$
\operatorname{det} A=\operatorname{det} A^{i} \cdot \operatorname{det} A^{e} \quad \text { (inner-outer factorization) }
$$

It thus follows from (8.15) that

$$
\begin{aligned}
\theta I_{n} \text { and } A \text { are left coprime } & \Longleftrightarrow \theta I_{n} \text { and } A^{i} \text { are left coprime } \\
& \Longleftrightarrow \theta \text { and } \operatorname{det} A^{i} \text { are coprime } \\
& \Longleftrightarrow \theta \text { and } \operatorname{det} A \text { are coprime }
\end{aligned}
$$


For right coprime-ness, we apply the above result and the fact that $\operatorname{det} \widetilde{A}=\widetilde{\operatorname{det} A}$.

\section{$\S$ 8.2. The model operator}

We recall that the model theorem (p. 6) states that if $T \in \mathcal{B}(\mathcal{H})$ is a contraction such that $\lim _{n \rightarrow \infty} T^{n} x=0$ for each $x \in \mathcal{H}$ (i.e., $T \in C_{0}$.), then there exists a unitary imbedding $V: \mathcal{H} \rightarrow H_{E}^{2}$ with $E:=\operatorname{cl} \operatorname{ran}\left(I-T T^{*}\right)$ such that $V \mathcal{H}=\mathcal{H}(\Delta)$ for some inner function $\Delta$ with values in $\mathcal{B}\left(E^{\prime}, E\right)$ and

$$
T=V^{*}\left(\left.S_{E}^{*}\right|_{\mathcal{H}(\Delta)}\right) V
$$

We may now ask what is a necessary and sufficient condition for $\operatorname{dim} E^{\prime}<\infty$ in the Model Theorem. In this section, we give a necessary condition for the finite-dimensionality of $E^{\prime}$.

For an inner function $\Delta$ with values in $\mathcal{B}\left(E^{\prime}, E\right)$, define

$$
H_{0}:=\left\{f \in \mathcal{H}(\Delta): \lim _{n \rightarrow \infty} P_{\mathcal{H}(\Delta)} S_{E}^{n} f=0\right\} .
$$

Then $H_{0}$ is a closed subspace of $\mathcal{H}(\Delta)$ and in this case, write

$$
E_{0}(\Delta):=\mathcal{H}(\Delta) \ominus H_{0} .
$$

Then $E_{0}(\Delta)$ is an invariant subspace of $S_{E}^{*}$, so that there exists an inner function $\Delta^{s} \in H^{\infty}\left(\mathcal{B}\left(E_{1}, E\right)\right)$ such that

$$
E_{0}(\Delta)=\mathcal{H}\left(\Delta^{s}\right)
$$

We then have:

Lemma 8.15. Let $\Delta$ be an inner function with values in $\mathcal{B}\left(E^{\prime}, E\right)$. Then

$$
\Delta=\Delta^{s} \Delta_{1}
$$

for some two-sided inner function $\Delta_{1}$ with values in $\mathcal{B}\left(E^{\prime}, E_{1}\right)$.

Proof. Observe that $H_{E}^{2}=\Delta H_{E^{\prime}}^{2} \oplus E_{0}(\Delta) \oplus H_{0}$. Thus,

$$
\Delta H_{E^{\prime}}^{2} \subseteq H_{E}^{2} \ominus E_{0}(\Delta)=\Delta^{s} H_{E_{1}}^{2}
$$

which implies that $\Delta=\Delta^{s} \Delta_{1}$ for some inner function $\Delta_{1}$ with values in $\mathcal{B}\left(E^{\prime}, E_{1}\right)$. We must show that $\Delta_{1}$ is two-sided. We first claim that

$$
f \in \Delta^{s} H_{E_{1}}^{2} \Longleftrightarrow\|f\|_{L_{E}^{2}}=\left\|\Delta^{*} f\right\|_{L_{E^{\prime}}^{2}}:
$$

indeed, since $\lim _{n \rightarrow \infty}\left\|\left(I_{E}-P_{+}\right) \Delta^{*} S_{E}^{n} f\right\|_{L_{E^{\prime}}^{2}}=0$ for each $f \in H_{E}^{2}$, a straightforward calculation shows that

$$
\lim _{n \rightarrow \infty}\left\|P_{\mathcal{H}(\Delta)} S_{E}^{n} f\right\|_{L_{E}^{2}}^{2}=\|f\|_{L_{E}^{2}}^{2}-\left\|\Delta^{*} f\right\|_{L_{E^{\prime}}^{2}}^{2}
$$

giving (8.19). Thus for all $x \in E_{1}$ with $\|x\|=1$,

$$
1=\left\|\Delta^{s} x\right\|_{L_{E}^{2}}=\left\|\Delta^{*} \Delta^{s} x\right\|_{L_{E^{\prime}}^{2}}=\left\|\Delta_{1}^{*} x\right\|_{L_{E^{\prime}}^{2}},
$$

which says that

$$
\int_{\mathbb{T}}\left\|\Delta_{1}^{*}(z) x\right\|^{2} d m(z)=1
$$


But since $\left\|\Delta_{1}^{*}(z) x\right\| \leq 1$, it follows that $\left\|\Delta_{1}^{*}(z) x\right\|=1$ a.e. on $\mathbb{T}$, so that $\Delta_{1}^{*}(z)$ is isometry for almost all $z \in \mathbb{T}$ and therefore $\Delta_{1}$ is two-sided inner. This completes the proof.

We then have:

Theorem 8.16. Let $T \in \mathcal{B}(H)$ be a contraction such that $\lim _{n \rightarrow \infty} T^{n} x=0$ for each $x \in H$ and have a characteristic function $\Delta$ with values in $\mathcal{B}\left(E^{\prime}, E\right)$. Then,

$$
\sup _{\zeta \in \mathbb{D}} \operatorname{dim}\left\{f(\zeta): f \in H_{0}\right\} \leq \operatorname{dim} E^{\prime},
$$

where $H_{0}$ is defined by (8.17). In particular, if $\operatorname{dim} E^{\prime}<\infty$, then $\max _{\zeta \in \mathbb{D}} \operatorname{dim}\{f(\zeta)$ : $\left.f \in H_{0}\right\}$ is finite.

Proof. It follows from $(\underline{8.18})$ and Lemma 8.15 that

$$
H_{0}=\mathcal{H}(\Delta) \ominus E_{0}(\Delta)=\mathcal{H}(\Delta) \ominus \mathcal{H}\left(\Delta^{s}\right) \subseteq \Delta^{s} H_{E^{\prime}}^{2} .
$$

We thus have

$$
\sup _{\zeta \in \mathbb{D}} \operatorname{dim}\left\{f(\zeta): f \in H_{0}\right\} \leq \sup _{\zeta \in \mathbb{D}} \operatorname{dim}\left\{\Delta^{s}(\zeta) g(\zeta): g \in H_{E^{\prime}}^{2}\right\}=\operatorname{dim} E^{\prime} .
$$

\section{$\S$ 8.3. An interpolation problem}

In the literature, many authors have considered the special cases of the following (scalar-valued or operator-valued) interpolation problem (cf. Co1, CHL2, CHL3, FF, Ga, Gu, GHR, HKL, HL1, HL2, [NT, Zh]).

Problem 8.17. For $\Phi \in L^{\infty}(\mathcal{B}(E))$, when does there exist a function $K \in$ $H^{\infty}(\mathcal{B}(E))$ with $\|K\|_{\infty} \leq 1$ satisfying

$$
\Phi-K \Phi^{*} \in H^{\infty}(\mathcal{B}(E)) ?
$$

If $\Phi$ is a matrix-valued rational function, this question reduces to the classical Hermite-Fejér interpolation problem.

For notational convenience, we write, for $\Phi \in L^{\infty}(\mathcal{B}(E))$,

$$
\mathcal{C}(\Phi):=\left\{K \in H^{\infty}(\mathcal{B}(E)): \Phi-K \Phi^{*} \in H^{\infty}(\mathcal{B}(E))\right\} .
$$

We then have:

Theorem 8.18. Let $\Phi \equiv \breve{\Phi}_{-}+\Phi_{+} \in L^{\infty}(\mathcal{B}(E))$. If $\mathcal{C}(\Phi)$ is nonempty then ker $H_{\Phi_{+}}^{*} \subseteq \operatorname{ker} H_{\Phi_{-}^{*}}^{*}$.

In particular,

$$
\operatorname{nc}\left\{\Phi_{+}\right\} \leq \operatorname{nc}\left\{\widetilde{\Phi_{-}}\right\}
$$


Proof. Suppose $\mathcal{C}(\Phi) \neq \emptyset$. Then there exists a function $K \in H^{\infty}(\mathcal{B}(E))$ such that $\Phi-K \Phi^{*} \in H^{\infty}(\mathcal{B}(E))$, then $H_{\Phi}=T_{\widetilde{K}}^{*} H_{\Phi^{*}}$, which implies that ker $H_{\Phi^{*}} \subseteq$ ker $H_{\Phi}$. But since $\Phi \equiv \breve{\Phi}_{-}+\Phi_{+} \in L^{\infty}(\mathcal{B}(E))$, it follows that

$$
H_{\Phi^{*}}=H_{\Phi_{+}^{*}}=H_{\Phi_{+}}^{*} \quad \text { and } \quad H_{\Phi}=H_{\breve{\Phi}_{-}}=H_{\Phi_{-}^{*}}^{*} .
$$

We thus have

$$
\operatorname{ker} H_{\Phi_{+}}^{*} \subseteq \operatorname{ker} H_{\Phi_{-}^{*}}^{*} .
$$

On the other hand, it follows from Lemma 4.4 that

$$
\Omega H_{E^{\prime}}^{2}=\operatorname{ker} H_{\overleftarrow{\Phi}_{+}}^{*} \subseteq \operatorname{ker} H_{\Phi_{-}^{*}}^{*}=\operatorname{ker} H_{\widetilde{\Phi}_{-}}^{*}=\Delta H_{E^{\prime \prime}}^{2}
$$

for some inner functions $\Omega$ and $\Delta$ with values in $\mathcal{B}\left(E^{\prime}, E\right)$ and $\mathcal{B}\left(E^{\prime \prime}, E\right)$, respectively. Thus $\Delta$ is a left inner divisor of $\Omega$, so that we have $\operatorname{dim} E^{\prime} \leq \operatorname{dim} E^{\prime \prime}$, which implies, by Theorem 4.10, that $\mathrm{nc}\left\{\Phi_{+}\right\} \leq \mathrm{nc}\left\{\widetilde{\Phi_{-}}\right\}$.

Corollary 8.19. Let $\Phi \equiv \breve{\Phi}_{-}+\Phi_{+} \in L^{\infty}(\mathcal{B}(E))$ and $\mathcal{C}(\Phi) \neq \emptyset$. If $\breve{\Phi}_{+}$is of bounded type, then $\Phi_{-}^{*}$ is of bounded type.

Proof. Suppose that $\Phi \equiv \breve{\Phi}_{-}+\Phi_{+} \in L^{\infty}(\mathcal{B}(E))$. Then by Lemma 3.13 $\Phi^{*}=\left(\breve{\Phi}_{-}\right)^{*}+\left(\Phi_{+}\right)^{*} \in L^{\infty}(\mathcal{B}(E))$. Thus $\left(\breve{\Phi}_{-}\right)^{*}$ is a strong $L^{2}$-function and so is $\Phi_{-}^{*}$. Assume that $\mathcal{C}(\Phi) \neq \emptyset$ and $\breve{\Phi}_{+}$is of bounded type. Then it follows from Theorem 8.18 and Lemma 4.4 that

$$
\Omega H_{E}^{2}=\operatorname{ker} H_{\Phi_{+}}^{*} \subseteq \operatorname{ker} H_{\Phi_{-}^{*}}^{*}=\Delta H_{E^{\prime \prime}}^{2}
$$

for some two-sided inner function $\Omega$ with values in $\mathcal{B}(E)$ and an inner function $\Delta$ with values in $\mathcal{B}\left(E^{\prime \prime}, E\right)$. Thus, $\Delta$ is a left inner divisor of $\Omega$ and hence, by Lemma 3.12, $\Delta$ is two-sided inner, so that $\Phi_{-}^{*}$ is of bounded type. 


\section{CHAPTER 9}

\section{Some unsolved problems}

In this paper we have explored the Beurling-Lax-Halmos Theorem and have tried to answer several outstanding questions. In this process, we have gotten interesting results on a canonical decomposition of strong $L^{2}$-functions, a connection between the Beurling degree and the spectral multiplicity, and the multiplicity-free model operators. However there are still open questions in which we are interested. In this chapter, we pose several unsolved problems.

\section{$\S$ 9.1. The Beurling degree of an inner matrix functions}

The theory of spectral multiplicity for operators of the class $C_{0}$ has been well developed (see [Ni1, Appendix 1], [SFBK]). For an inner matrix function $\Delta \in$ $H_{M_{N}}^{\infty}$ and $k=0,1, \cdots, N$, let

$$
\delta_{k}:=\text { g.c.d. }\{\text { all inner parts of the minors of order } N-k \text { of } \Delta\} .
$$

Then it is well-known that if $T \in C_{0}$ with characteristic function $\Delta \in H_{M_{N}}^{\infty}$, then

$$
\mu_{T}=\min \left\{k: \delta_{k}=\delta_{k+1}\right\} .
$$

In fact, the proof for " $\geq$ " in (9.2) is not difficult. But the proof for " $\leq$ " is so complicated. However, Theorem 6.6 gives a simple proof for " $\leq$ " in $(9.2)$ with the aid of the Moore-Nordgren Theorem. To see this, we recall that for an inner function $\Delta_{k}(k=1,2)$ with values in $M_{N}, \Delta_{1}$ and $\Delta_{2}$ are called quasi-equivalent if there exist functions $X, Y \in H_{M_{N}}^{\infty}$ such that $X \Delta_{1}=\Delta_{2} Y$ and such that the inner parts $(\operatorname{det} X)^{i}$ and $(\operatorname{det} Y)^{i}$ of the corresponding determinants are coprime to $\left(\operatorname{det} \Delta_{k}\right)^{i}(k=1,2)$.

The following theorem shows that the spectral multiplicity of $C_{0}$-operators with square-inner characteristic functions can be computed by studying diagonal characteristic functions (cf. [No, $\mathbf{M N}$, [Ni1):

\section{Nordgren-Moore Theorem.}

(a) Let $\Delta_{k}(k=1,2)$ be an inner function with values in $M_{N}$ and let $T_{k}:=$ $\left.P_{\mathcal{H}(\Delta)} S_{\mathbb{C}^{N}}\right|_{\mathcal{H}\left(\Delta_{k}\right)}(k=1,2)$. If $\Delta_{1}$ and $\Delta_{2}$ are quasi-equivalent then $\mu_{T_{1}}=\mu_{T_{2}}$.

(b) Let $\Delta$ be an inner function with values in $M_{N}$. Then $\Delta$ is quasi-equivalent to a unique diagonal inner function

$$
\operatorname{diag}\left(\delta_{0} / \delta_{1}, \delta_{1} / \delta_{2}, \cdots, \delta_{N-1} / \delta_{N}\right)
$$


By the Nordgren-Moore Theorem (a) and Theorem 6.6 we can see that if $\Delta_{1}$ and $\Delta_{2}$ are quasi-equivalent square inner matrix functions then

$$
\operatorname{deg}_{B}\left(\widetilde{\Delta}_{1}\right)=\operatorname{deg}_{B}\left(\widetilde{\Delta}_{2}\right) .
$$

We now have:

Proposition 9.1. If $\Delta$ is an $N \times N$ square-inner matrix function then

$$
\operatorname{deg}_{B}(\Delta) \leq \min \left\{k: \delta_{k}=\delta_{k+1}\right\} .
$$

Proof. Let $m:=\min \left\{k: \delta_{k}=\delta_{k+1}\right\}$. Then by the Nordgren-Moore Theorem, $\Delta$ is quasi-equivalent to $\Theta \equiv \operatorname{diag}\left(\delta_{0} / \delta_{1}, \cdots, \delta_{m-1} / \delta_{m}, 1, \cdots, 1\right)$. We now take

$$
\Phi:=\left[\begin{array}{cccc}
\delta_{0} / \delta_{1} & 0 & \cdots & 0 \\
0 & \delta_{1} / \delta_{2} & & \vdots \\
\vdots & & \ddots & 0 \\
0 & \cdots & 0 & \delta_{m-1} / \delta_{m} \\
0 & \cdots & 0 & 1 \\
& \vdots & & \vdots \\
0 & \cdots & 0 & 1
\end{array}\right] \in H_{M_{N \times m}}^{\infty} .
$$

Then a direct calculation shows that

$$
\operatorname{ker} H_{\Phi^{*}}=\left(\sum_{k=1}^{m} \bigoplus\left(\delta_{k-1} / \delta_{k}\right) H^{2}\right) \bigoplus H_{\mathbb{C}^{N-m}}^{2}=\Theta H_{\mathbb{C}^{n}}^{2}
$$

It thus follows from (6.7) and (9.3) that $\operatorname{deg}_{B}(\Delta)=\operatorname{deg}_{B}(\Theta) \leq m$.

Corollary 9.2. If $\Theta$ is a diagonal inner matrix function of the form $\Theta:=$ $\operatorname{diag}\left(\theta_{1}, \cdots, \theta_{N}\right)$ (where each $\theta_{i}$ is a scalar inner function) then

$$
\operatorname{deg}_{B}(\Theta)=\max \operatorname{card}\left\{\sigma: \sigma \subseteq\{1, \cdots, N\}, \text { g.c.d. }\left\{\theta_{i}: i \in \sigma\right\} \neq 1\right\} .
$$

Proof. This follows at once from (9.2) and Theorem 6.6.

Now Proposition 9.1 together with Theorem 6.6 gives a simple proof for " $\leq$ " in (9.2). Consequently, in (9.4), we may take "=" in place of " $\leq$ ". However we were unable to derive a similar formula to (9.4) for non-square inner matrix function. Thus we would like to pose:

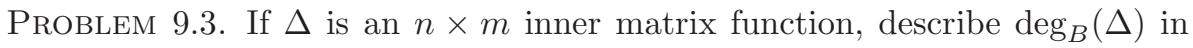
terms of its entries (e.g., minors).

\section{$\S$ 9.2. Spectra of model operators}

We recall that if $\theta$ is a scalar inner function, then we may write

$$
\theta(\zeta)=B(\zeta) \exp \left(-\int_{\mathbb{T}} \frac{z+\zeta}{z-\zeta} d \mu(z)\right),
$$


where $B$ is a Blaschke product and $\mu$ is a singular measure on $\mathbb{T}$ and that the spectrum, $\sigma(\theta)$, of $\theta$ is defined by

$$
\sigma(\theta):=\left\{\lambda \in \operatorname{cl} \mathbb{D}: \frac{1}{\theta} \text { can be continued analytically into a neighborhood of } \lambda\right\} .
$$

Then it was ([Ni1, p.63]) known that the spectrum $\sigma(\theta)$ of $\theta$ is given by

$$
\sigma(\theta)=\operatorname{cl} \theta^{-1}(0) \bigcup \operatorname{supp} \mu \text {. }
$$

It was also (cf. [Ni1, p.72]) known that if $\left.T \equiv P_{\mathcal{H}(\Delta)} S_{E}\right|_{\mathcal{H}(\Delta)} \in C_{0}$, then

$$
\sigma(T)=\sigma\left(m_{\Delta}\right) .
$$

In view of (9.6), we may ask what is the spectrum of the model operator $\left.S_{E}^{*}\right|_{\mathcal{H}(\Delta)}$

? Here is an answer.

Proposition 9.4. Let $T:=\left.S_{E}^{*}\right|_{\mathcal{H}(\Delta)}$ for an inner function $\Delta$ with values in $\mathcal{B}(D, E)$. If $\Delta$ has a meromorphic pseudo-continuation of bounded type in $\mathbb{D}^{e}$ and $\omega_{\Delta}$ is the pseudo-characteristic scalar inner function of $\Delta$, then

$$
\overline{\sigma\left(\omega_{\Delta}\right)} \subseteq \sigma(T) .
$$

Proof. If $\Delta_{c}$ is the complementary factor, with values in $\mathcal{B}\left(D^{\prime}, E\right)$, of $\Delta$, then by the proof of Lemma 7.5, $\left[\Delta, \Delta_{c}\right]$ is two-sided inner and has a meromorphic pseudo-continuation of bounded type in $\mathbb{D}^{e}$. Thus, by Proposition 4.29] $\left.S_{E}^{*}\right|_{\mathcal{H}\left(\left[\Delta, \Delta_{c}\right]\right)}$ belongs to $C_{0}$. Then by the Model Theorem, we have

$$
\left.\left.S_{E}^{*}\right|_{\mathcal{H}\left(\left[\Delta, \Delta_{c}\right]\right)} \cong P_{\mathcal{H}\left(\left[\widetilde{\left.\Delta, \Delta_{c}\right]}\right)\right.} S_{E}\right|_{\mathcal{H}\left(\left[\widetilde{\Delta, \Delta_{c}}\right]\right)} .
$$

It thus follows from Lemma 7.5 and (9.6) that

$$
\sigma\left(\left.S_{E}^{*}\right|_{\mathcal{H}\left(\left[\Delta, \Delta_{c}\right]\right.}\right)=\sigma\left(m_{\left[\widetilde{\left.\Delta, \Delta_{c}\right]}\right.}\right)=\sigma\left(\widetilde{\omega_{\Delta}}\right)=\overline{\sigma\left(\omega_{\Delta}\right)} .
$$

On the other hand, observe

$$
\left[\Delta, \Delta_{c}\right] H_{D \oplus D^{\prime}}^{2}=\Delta H_{D}^{2} \oplus \Delta_{c} H_{D^{\prime}}^{2},
$$

and hence

$$
\mathcal{H}(\Delta)=\mathcal{H}\left(\left[\Delta, \Delta_{c}\right]\right) \oplus \Delta_{c} H_{D^{\prime}}^{2}
$$

Thus we may write

$$
T=\left[\begin{array}{cc}
T_{1} & * \\
0 & T_{2}
\end{array}\right]:\left[\begin{array}{c}
\mathcal{H}\left(\left[\Delta, \Delta_{c}\right]\right) \\
\Delta_{c} H_{D^{\prime}}^{2}
\end{array}\right] \rightarrow\left[\begin{array}{c}
\mathcal{H}\left(\left[\Delta_{,}, \Delta_{c}\right]\right) \\
\Delta_{c} H_{D^{\prime}}^{2}
\end{array}\right] .
$$

Note that $T_{1}=\left.S_{E}^{*}\right|_{\mathcal{H}\left(\left[\Delta, \Delta_{c}\right]\right)}$. Since by (9.5) and (9.8), $\sigma\left(T_{1}\right)$ has no interior points, so that $\sigma\left(T_{1}\right) \cap \sigma\left(T_{2}\right)$ has no interior points. Thus we have $\sigma(T)=\sigma\left(T_{1}\right) \cup \sigma\left(T_{2}\right)$ because in the Banach space setting, the passage from $\sigma\left(\begin{array}{cc}A & C \\ 0 & B\end{array}\right)$ to $\sigma(A) \cup \sigma(B)$ is the filling in certain holes in $\sigma\left(\begin{array}{cc}A & C \\ 0 & B\end{array}\right)$, occurring in $\sigma(A) \cap \sigma(B)$ (cf. [HLL). Therefore, by (9.8), we have $\overline{\sigma\left(\omega_{\Delta}\right)} \subseteq \sigma(T)$.

We would like to pose:

Problem 9.5. If $T:=\left.S_{E}^{*}\right|_{\mathcal{H}(\Delta)}$ for an inner function $\Delta$ having a meromorphic pseudo-continuation of bounded type in $\mathbb{D}^{e}$, describe the spectrum of $T$ in terms of the pseudo-characteristic scalar inner function of $\Delta$. 


\section{$\S$ 9.3. The spectral multiplicity of model operators}

It was known (cf. [Ni1, p. 41]) that if $T:=\left.S_{E}^{*}\right|_{\mathcal{H}(\Delta)}$ for an inner function $\Delta$ with values in $\mathcal{B}\left(E^{\prime}, E\right)$, with $\operatorname{dim} E^{\prime}<\infty$, then $\mu_{T} \leq \operatorname{dim} E^{\prime}+1$. Theorem 7.24 says that if $\Delta$ has a meromorphic pseudo-continuation of bounded type in $\mathbb{D}^{e}$, then

$$
\mu_{T} \leq \operatorname{dim} E^{\prime} .
$$

However we were unable to find an example showing that Theorem 7.24 may fail if the condition " $\Delta$ has a meromorphic pseudo-continuation of bounded type in $\mathbb{D}^{e}$ " is dropped. Thus we would like to pose:

Problem 9.6. Find an example of the operator $\left.T \equiv S_{E}^{*}\right|_{\mathcal{H}(\Delta)}$ for an inner function $\Delta$ with values in $\mathcal{B}\left(E^{\prime}, E\right)$, with $\operatorname{dim} E^{\prime}<\infty$, satisfying

$$
\mu_{T}=\operatorname{dim} E^{\prime}+1 \text {. }
$$

\section{$\S$ 9.4. The Model Theorem}

The Model Theorem (cf. p. 6) says that if $T \in C_{0}$., i.e., $T \in \mathcal{B}(\mathcal{H})$ is a contraction such that $\lim _{n \rightarrow \infty} T^{n} x=0$ for each $x \in H$, then $T$ is unitarily equivalent to the truncated backward shift $\left.S_{E}^{*}\right|_{\mathcal{H}(\Delta)}$ with the characteristic function $\Delta$ with values in $\mathcal{B}\left(E^{\prime}, E\right)$, where

$$
E=\operatorname{cl} \operatorname{ran}\left(I-T^{*} T\right) .
$$

However, we were unable to determine $E^{\prime}$ in terms of spectral properties of $T$ as in (9.10). In Theorem 8.16, we give a necessary condition for "dim $E^{\prime}<\infty$." Thus, we would like to pose:

Problem 9.7. Let $T \in C_{0}$. and $\Delta \in H^{\infty}\left(\mathcal{B}\left(E^{\prime}, E\right)\right)$ be the characteristic function of $T$. For which operator $T$, we have $\operatorname{dim} E^{\prime}<\infty$ ?

\section{$\S$ 9.5. Cowen's Theorem and Abrahamse's Theorem}

For $\Phi \in L^{\infty}(\mathcal{B}(E))$, write

$$
\mathcal{E}(\Phi):=\left\{K \in H^{\infty}(\mathcal{B}(E)): \Phi-K \Phi^{*} \in H^{\infty}(\mathcal{B}(E)) \text { and }\|K\|_{\infty} \leq 1\right\},
$$

i.e., $\mathcal{E}(\Phi)=\left\{K \in \mathcal{C}(\Phi):\|K\|_{\infty} \leq 1\right\}$ (cf. p 81). If $\operatorname{dim} E=1$ and $\Phi \equiv \varphi$ is a scalar-valued function then an elegant theorem of C. Cowen (cf. Co1, NT, CL ) says that $\mathcal{E}(\varphi)$ is nonempty if and only if $T_{\varphi}$ is hyponormal, i.e., the selfcommutator $\left[T_{\varphi}^{*}, T_{\varphi}\right]$ is positive semi-definite. Cowen's Theorem is to recast the operator-theoretic problem of hyponormality into the problem of finding a solution of an interpolation problem. In GHR, it was shown that the Cowen's theorem still holds for a Toeplitz operator $T_{\Phi}$ with a matrix-valued normal (i.e., $\Phi^{*} \Phi=\Phi \Phi^{*}$ ) symbol $\Phi \in L_{M_{n}}^{\infty}$.

We then have: 
Problem 9.8. Extend Cowen's theorem for a Toeplitz operator with an operatorvalued normal symbol $\Phi \in L^{\infty}(\mathcal{B}(E))$.

We recall that an operator $T \in \mathcal{B}(\mathcal{H})$ is called subnormal if $T$ has a normal extension, i.e., $T=\left.N\right|_{\mathcal{H}}$, where $N$ is a normal operator on some Hilbert space $\mathcal{K} \supseteq \mathcal{H}$ such that $\mathcal{H}$ is invariant for $N$. In 1979, P.R. Halmos posed the following problem, listed as Problem 5 in his Lecture "Ten problems in Hilbert space" ([Ha2, [Ha3): Is every subnormal Toeplitz operator $T_{\varphi}$ with symbol $\varphi \in L^{\infty}$ either normal or analytic (i.e., $\left.\varphi \in H^{\infty}\right)$ ? In 1984, C. Cowen and J. Long CoL have answered this question in the negative. To date, a characterization of subnormality of Toeplitz operators $T_{\varphi}$ in terms of the symbols $\varphi$ has not been found. The best partial answer to Halmos' Problem 5 was given by M.B. Abrahamse: If $\varphi \in L^{\infty}$ is such that $\varphi$ or $\bar{\varphi}$ is of bounded type, then $T_{\varphi}$ is either normal or analytic; this is called Abrahamse's Theorem. Very recently, in CHL3, Theorem 7.3], Abrahamse's Theorem was extended to the cases of Toeplitz operators $T_{\Phi}$ with matrix-valued symbols $\Phi$ under some constraint on the symbols $\Phi$; concretely, when " $\Phi$ has a tensored-scalar singularity."

We would like to pose:

Problem 9.9. Extend Abrahamse's Theorem to Toeplitz operators $T_{\Phi}$ with operator-valued symbols $\Phi \in L^{\infty}(\mathcal{B}(E))$. 



\section{Bibliography}

[Ab] M.B. Abrahamse, Subnormal Toeplitz operators and functions of bounded type, Duke Math. J. 43 (1976), 597-604.

[ADR] D. Alpay, A. Dijksma, and J. Rovnyak, A theorem on Beurling-Lax type for Hilbert spaces of functions analytic in the unit ball, Integral Equations Operator Theory $\mathbf{4 7}$ (2003), 251-274.

[AS] D. Alpay and I. Sabodini, Beurling-Lax type theorems in the complex and quaternionic setting, Linear Alg. Appl. 530 (2017), 15-46.

[BH1] J.A. Ball and J.W. Helton, A Beurling-Lax theorem for the Lie group $U(m, n)$ which contains most classical interpolation theory, J. Operator Theory 9 (1983), 107-142.

[BH2] J.A. Ball and J.W. Helton, Beurling-Lax representations using classical Lie groups with many applications. III. Groups preserving two bilinear forms, Amer. J. Math. 108 (1986), 95-174.

[BH3] J.A. Ball and J.W. Helton, Shift invariant manifolds and nonlinear analytic function theory, Integral Equations Operator Theory 11 (1988), 615-725.

[Ber] H. Bercovici, Operator Theory and Arithmetic in $H^{\infty}$, Mathematical Surveys and Monographs, vol. 26, Amer. Math. Soc., Providence, 1988.

[Beu] A. Beurling, On two problems concerning linear transformations in Hilbert space, Acta Math. 81 (1949), 239-255.

[BB] S. Bochner and H.F. Bohnenblust, Analytic functions with almost periodic coefficients, Ann. Math. 35 (1934), 152-161.

[BS] A. Böttcher and B. Silbermann, Analysis of Toeplitz Operators, Springer, BerlinHeidelberg, 2006.

[Ca] M. Carlsson, On the Beurling-Lax theorem for domains with one hole, New York J. Math. 17A (2011), 193-212.

[Con] J.B. Conway, A Course in Functional Analysis, Springer, New York, 1990.

[Co1] C. Cowen, Hyponormality of Toeplitz operators, Proc. Amer. Math. Soc. 103 (1988), 809-812.

[Co2] C. Cowen, Hyponormal and subnormal Toeplitz operators, Surveys of Some Recent Results in Operator Theory, I (J.B. Conway and B.B. Morrel, eds.), Pitman Research Notes in Mathematics, Vol 171 (1988), 155-167.

[CoL] C. Cowen and J. Long, Some subnormal Toeplitz operators, J. Reine Angew. Math. 351 (1984), 216-220.

[CHKL] R.E. Curto, I.S. Hwang, D. Kang and W.Y. Lee, Subnormal and quasinormal Toeplitz operators with matrix-valued rational symbols, Adv. Math. 255 (2014), 561-585.

[CHL1] R.E. Curto, I.S. Hwang and W.Y. Lee, Which subnormal Toeplitz operators are either normal or analytic?, J. Funct. Anal. 263(8) (2012), 2333-2354.

[CHL2] R.E. Curto, I.S. Hwang and W.Y. Lee, Hyponormality and subnormality of block Toeplitz operators, Adv. Math. 230 (2012), 2094-2151.

[CHL3] R.E. Curto, I.S. Hwang and W.Y. Lee, Matrix functions of bounded type: An interplay between function theory and operator theory, Mem. Amer. Math. Soc. 260 (2019), no. 1253 , vi+100.

[CL] R.E. Curto and W.Y. Lee, Joint hyponormality of Toeplitz pairs, Mem. Amer. Math. Soc. 150 (2001), no. 712 , x+65.

[dR] L. de Branges and J. Rovnyak, Square Summable Power Series, Holt, Rinehart and Winston, New York-Toronto, 1966.

[Do1] R.G. Douglas, Banach Algebra Techniques in Operator Theory, Academic Press, New York, 1972 . 
[Do2] R.G. Douglas, Banach Algebra Techniques in the Theory of Toeplitz Operators, CBMS vol. 15, Amer. Math. Soc., Providence, 1973.

[DSS] R.G. Douglas, H. Shapiro, and A. Shields, Cyclic vectors and invariant subspaces for the backward shift operator, Ann. Inst. Fourier(Grenoble) 20 (1970), 37-76.

[DS] N. Dunford and J. Schwartz, Linear Operators. General theory, Part I., New York, Wiley, 1958.

[Du] P.L. Duren, Theory of $H^{p}$ spaces, New York, Academic Press, 1970.

[FF] C. Foiaş and A. Frazho, The Commutant Lifting Approach to Interpolation Problems, Oper. Th. Adv. Appl. vol. 44, Birkhäuser, Boston, 1993.

[FB] A. Frazho and W. Bhosri, An Operator Perspective on Signals and Systems, Oper. Th. Adv. Appl. vol. 204, Birkhäuser, Basel, 2010.

[Fu1] P. Fuhrmann, On Hankel operator ranges, meromorphic pseudo-continuation and factorization of operator-valued analytic functions, J. London Math. Soc.(2), 13 (1975), 323-327.

[Fu2] P. Fuhrmann, Linear Systems and Operators in Hilbert Spaces, McGraw-Hill, New York, 1981.

[Ga] J. Garnett, Bounded Analytic Functions, Academic Press, New York, 1981.

[Go] S. Goldberg, Unbounded Linear Operators, Dover, New York, 2006.

[Gu] C. Gu, A generalization of Cowen's characterization of hyponormal Toeplitz operators, J. Funct. Anal. 124 (1994), 135-148.

[GHR] C. Gu, J. Hendricks and D. Rutherford, Hyponormality of block Toeplitz operators, Pacific J. Math. 223 (2006), 95-111.

[Ha1] P.R. Halmos, Shifts on Hilbert spaces, J. Reine Angew. Math. 208 (1961), 102-112.

[Ha2] P.R. Halmos, Ten problems in Hilbert space, Bull. Amer. Math. Soc. 76 (1970), 887-933.

[Ha3] P.R. Halmos, Ten years in Hilbert space, Integral Equations Operator Theory 2 (1979), 529-564.

[Ha4] P.R. Halmos, A Hilbert Space Problem Book, Springer, New York, 1982.

[HLL] J.K. Han, H.Y. Lee, and W.Y. Lee, Invertible completions of $2 \times 2$ triangular operator matrices. Proc. Amer. Math. Soc. 128, 119-123.

[Hed] H. Hedenmalm, A factorization theorem for square area-integrable analytic functions, J. Reine Angew. Math. 422 (1991), 45-68.

[Hel] H. Helson, Lectures on Invariant Subspaces, Academic Press, New York, 1964.

[HP] E. Hille and R.S. Phillips, Functional Analysis and Semi-groups, Amer. Math. Soc. Coll. Publ. 31, Providence, 1957.

[Ho] K. Hoffman, Banach Spaces of Analytic functions, Englewood Cliffs, Prentice-Hall, 1962.

[HKL] I.S. Hwang, I.H. Kim, and W.Y. Lee, Hyponormality of Toeplitz operators with polynomial symbols, Math. Ann. 313(2) (1999), 247-261.

[HL1] I.S. Hwang and W.Y. Lee, Hyponormality of trigonometric Toeplitz operators, Trans. Amer. Math. Soc. 354 (2002), 2461-2474.

[HL2] I.S. Hwang and W.Y. Lee, Hyponormality of Toeplitz operators with rational symbols, Math. Ann. 335 (2006), 405-414.

[Lax] P.D. Lax, Translation invariant subspaces, Acta Math. 101 (1959), 163-178.

[MR] R. A. Martínez-Avendaño and P. Rosenthal, An Introduction to Operators on the HardyHilbert Space, Springer, New York, 2007.

[MN] B. Moore III and E.A. Nordgren, On quasi-equivalence and quasi-similarity, Acta. Sci. Math. (Szeged) 34 (1973), 311-316.

[NT] T. Nakazi and K. Takahashi, Hyponormal Toeplitz operators and extremal problems of Hardy spaces, Trans. Amer. Math. Soc. 338 (1993), 753-767.

[Ni1] N.K. Nikolskii, Treatise on the Shift Operator, Springer, New York, 1986.

[Ni2] N.K. Nikolskii, Operators, Functions, and Systems: An Easy Reading Volume I: Hardy, Hankel, and Toeplitz, Mathematical Surveys and Monographs, vol. 92, Amer. Math. Soc., Providence, 2002.

[No] E.A. Nordgren, On quasi-equivalence of matrices over $H^{\infty}$, Acta. Sci. Math. (Szeged) 34 (1973), 301-310.

[Pe] V.V. Peller, Hankel Operators and Their Applications, Springer, New York, 2003.

[Po] V.P. Potapov, The multiplicative structure of J-contractive matrix functions, Amer. Math. Soc. Transl. 15(2) (1960), 131-243. 
[Ri] S. Richter, A representation theorem for cyclic analytic two-isometries, Trans. Amer. Math. Soc. 328(1) (1991), 325-349.

[Sa] D. Sarason, Sub-Hardy Hilbert Spaces in the Unit Disk, John Wiley \& Sons, Inc., New York, 1994.

[Sh] H.S. Shapiro, Generalized analytic continuation, In: Symposia on Theoretical Physics and Mathematics, vol. 8, pp. 151-163, Plenum Press, 1968.

[SFBK] B. Sz.-Nagy, C. Foiaş, H. Bercovici, and L. Kérchy, Harmonic Analysis of Operators on Hilbert Space, Springer, New York, 2010.

[VN] V.I. Vasyunin and N.K. Nikolskii, Classification of $H^{2}$-functions according to the degree of their cyclicity, Math. USSR Izvestiya 23(2) (1984), 225-242.

[Wo] W.R. Wogen, On some operators with cyclic vectors, Indiana Univ. Math. J. 27 (1978), 163-171.

[Zh] K. Zhu, Hyponormal Toeplitz operators with polynomial symbols, Integral Equations Operator Theory 21 (1996), 376-381. 